\title{
The Jupiter Energetic Particle Detector Instrument (JEDI) Investigation for the Juno Mission
}

\author{
B.H. Mauk • D.K. Haggerty • S.E. Jaskulek • C.E. Schlemm • L.E. Brown • \\ S.A. Cooper · R.S. Gurnee · C.M. Hammock • J.R. Hayes • G.C. Ho · J.C. Hutcheson • \\ A.D. Jacques • S. Kerem • C.K. Kim • D.G. Mitchell • K.S. Nelson • C.P. Paranicas • \\ N. Paschalidis $\cdot$ E. Rossano $\cdot$ M.R. Stokes
}

Received: 26 February 2013 / Accepted: 16 September 2013 / Published online: 26 November 2013

(C) The Author(s) 2013. This article is published with open access at Springerlink.com

\begin{abstract}
The Jupiter Energetic Particle Detector Instruments (JEDI) on the Juno Jupiter polar-orbiting, atmosphere-skimming, mission to Jupiter will coordinate with the several other space physics instruments on the Juno spacecraft to characterize and understand the space environment of Jupiter's polar regions, and specifically to understand the generation of Jupiter's powerful aurora. JEDI comprises 3 nearly-identical instruments and measures at minimum the energy, angle, and ion composition distributions of ions with energies from $\mathrm{H}: 20 \mathrm{keV}$ and $\mathrm{O}: 50 \mathrm{keV}$ to $>1 \mathrm{MeV}$, and the energy and angle distribution of electrons from $<40$ to $>500 \mathrm{keV}$. Each JEDI instrument uses microchannel plates (MCP) and thin foils to measure the times of flight (TOF) of incoming ions and the pulse height associated with the interaction of ions with the foils, and it uses solid state detectors (SSD's) to measure the total energy $(E)$ of both the ions and the electrons. The MCP anodes and the SSD arrays are configured to determine the directions of arrivals of the incoming charged particles. The instruments also use fast triple coincidence and optimum shielding to suppress penetrating background radiation and incoming UV foreground. Here we describe the science objectives of JEDI, the science and measurement requirements, the challenges that the JEDI team had in meeting these requirements, the design and operation of the JEDI instruments, their calibrated performances, the JEDI inflight and ground operations, and the initial measurements of the JEDI instruments in interplanetary space following the Juno launch on 5 August 2011. Juno will begin its prime science operations, comprising 32 orbits with dimensions $1.1 \times 40$ $\mathrm{RJ}$, in mid-2016.
\end{abstract}

Keywords Jupiter · magnetosphere · Aurora · Juno Mission · Radiation belts · Energetic Particles

B.H. Mauk $(\varangle)$ · D.K. Haggerty · S.E. Jaskulek · C.E. Schlemm · L.E. Brown · S.A. Cooper ·

R.S. Gurnee - J.R. Hayes - G.C. Ho - J.C. Hutcheson - S. Kerem · C.K. Kim - D.G. Mitchell •

K.S. Nelson · C.P. Paranicas · M.R. Stokes

The Johns Hopkins University, Applied Physics Laboratory, Laurel, MD, USA

e-mail: barry.mauk@jhuapl.edu

C.M. Hammock · A.D. Jacques - N. Paschalidis · E. Rossano

Goddard Space Flight Center, NASA (National Aeronautics and Space Administration), Greenbelt,

MD, USA 


\section{Introduction and Background}

The purpose of the Jupiter Energetic Particle Detector Instrument (JEDI) on the Juno polarorbiting, atmosphere-skimming, mission to Jupiter is to coordinate with the several other space physics instruments on Juno to characterize and understand the space environment of Jupiter's polar regions, and specifically to understand the generation of Jupiter's powerful aurora. JEDI measures the energetic component of electrons and ions that:

- participate in the generation of Jupiter's aurora,

- heat and ionize the upper atmosphere of Jupiter, and

- contain signatures of the structure of Jupiter's space environment, particularly the inner magnetosphere.

Specifically, JEDI is required to measure the energy, angle, and ion composition distributions of ions with energies from $\mathrm{H}: 20 \mathrm{keV}$ and $\mathrm{O}: 50 \mathrm{keV}$ to $>1 \mathrm{MeV}$, and the energy and angle distribution of electrons from $<40$ to $>500 \mathrm{keV}$. JEDI uses microchannel plates (MCP) and thin foils to measure the time of flight (TOF) and MCP pulse height of the incoming ions, and it uses solid state detectors (SSD's) to measure the total energy (E) of both the ions and the electrons.

The overall characteristics and scientific purposes of the Juno mission, including the objectives of understanding Jupiter's origin and internal structure, understanding the generation of Jupiter's powerful magnetic field, targeting atmospheric structure and dynamics, determining the water content of Jupiter's atmosphere, in addition to understanding Jupiter's polar space environment, are described by Bolton et al. (2013, this issue). The scientific objectives, rationale, and implementation of Juno's objective of understanding Jupiter's polar space environment and aurora are described by Bagenal et al. (2013, this issue). Some of the outstanding science issues associated with comparing aurora at all of the strongly magnetized planets are discussed by Mauk and Bagenal (2012) and in other articles in the American Geophysical Union Geophysical Monograph volume where that article appears (Keiling et al. 2012)

The uniqueness of the Juno mission for targeting Jupiter's auroral and polar environment are made clear in Fig. 1. Juno will visit precisely where auroral particles are thought to be energized and will make the detailed connection between the structure and energetics of Jupiter's aurora and the detailed particle characteristics and electromagnetic conditions that create the aurora.

Here we describe detailed characteristics of the JEDI instrument which works with the MAG, JADE, WAVES, UVIS, and JIRAM instruments described elsewhere in this issue to characterize and understand Jupiter's aurora and polar regions.

\section{JEDI Requirements}

\subsection{Program Level Requirements that Drive JEDI's Design}

The selected Program Level (Level-1) Science Objectives that address Juno's science objectives for Jupiter's Polar Regions are listed here. Specifically, Juno will:

(1) Investigate the primary auroral processes responsible for particle acceleration.

(2) Characterize the field-aligned currents that transfer angular momentum from Jupiter to its magnetosphere.

(3) Identify and characterize auroral radio and plasma wave emissions associated with particle acceleration. 

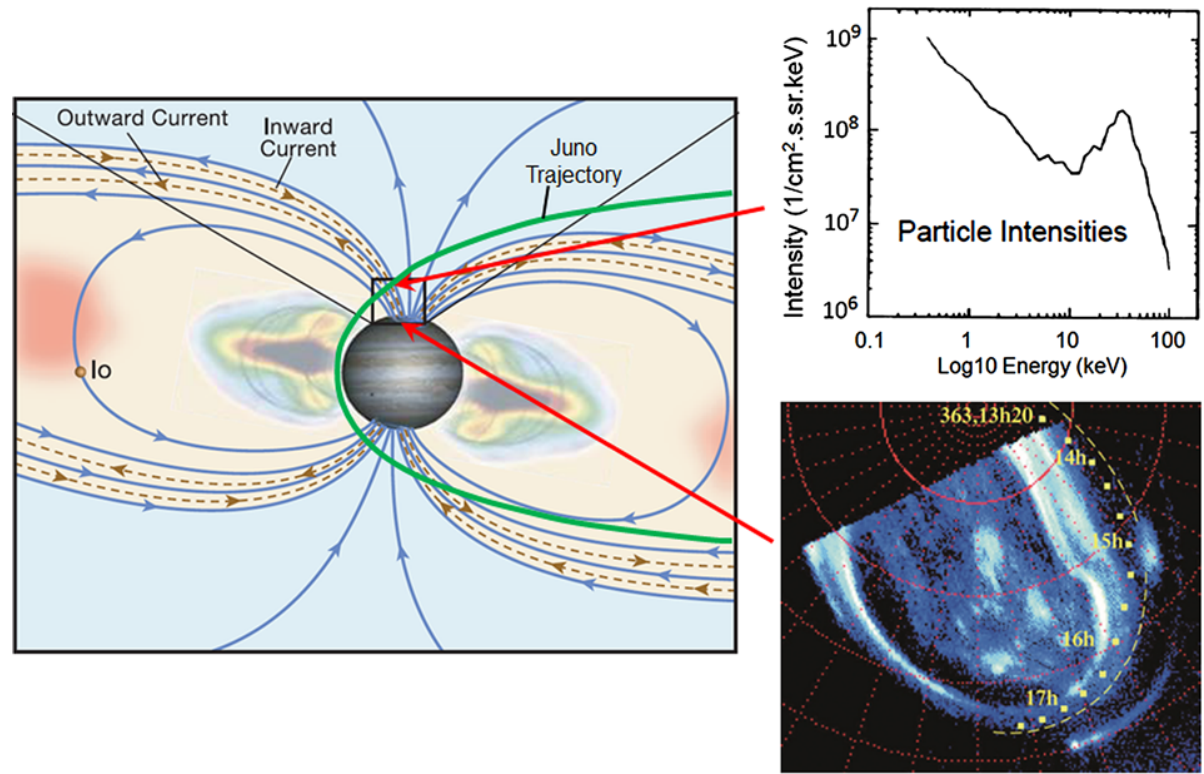

Fig. 1 Juno spacecraft near-Jupiter spacecraft orbit showing the ability of the Juno mission to make the connection between particle acceleration processes (upper right; Earth auroral spectrum from Arnoldy 1981) and the resulting auroral emissions (lower right; Hubble imaged published by (Mauk et al. 2002))

(4) Characterize the nature and spatial scale of auroral features.

JEDI is most relevant to Science Objective numbers 1, 3, and 4.

Within the same document, the selected Program Level (Level-1) Science Requirements that address Juno's requirement to characterized Jupiter's Polar Region is described here. Specifically, Juno must:

- Measure fields and charged particles (ions and electrons) in Jupiter's polar magnetosphere and obtain UV images of auroral emissions to survey and explore Jupiter's threedimensional polar magnetosphere.

JEDI measures the high energy component of the ions and electrons described within this requirement in conjunction with the requirements of the JADE instrument to measure the lower energy components

\subsection{JEDI-Relevant Mission Level Requirements (Level 2)}

The Juno Mission-Level requirements (Level 2) that flow down from the Program-Level requirements described above are:

- L2-PS-725. The Juno Project shall measure the pitch angle and energy distribution of electrons over both Jovigraphic polar regions over all science orbits.

- L2-PS-726. The Juno Project shall measure the time-variable, pitch angle, energy, and composition distributions of ions in the polar magnetosphere over both Jovigraphic polar regions over all science orbits. 
Table 1 JEDI Level-3/Level-4 Performance Requirements

\begin{tabular}{|c|c|c|c|}
\hline Parameter & Required & Capability & Comment \\
\hline Electron Energies & $40-500 \mathrm{keV}$ & $25-1000 \mathrm{keV}$ & Abuts JADE \\
\hline $\begin{array}{l}\text { Ion Energies } \\
\text { (Measured, not } \\
\text { discriminated) }\end{array}$ & $\begin{array}{l}\mathrm{H}: 20-1000 \mathrm{keV} \\
\mathrm{He}: 30-1000 \\
\mathrm{O}: 50-1000\end{array}$ & $\begin{array}{l}\mathrm{H}+10-2000 \mathrm{keV} \\
\mathrm{He}: 25-2000 \\
\mathrm{O} / \mathrm{S}+45-10000 \mathrm{keV}\end{array}$ & Abuts JADE \\
\hline $\begin{array}{l}\text { Energy } \\
\text { Resolution }\end{array}$ & $\begin{array}{l}\text { Ions }<25 \%(<30 \% \text { for } \\
\mathrm{E}<40 \mathrm{keV}) ; \text { Electron }< \\
\text { larger of } 25 \% \text { and } 15 \mathrm{keV}\end{array}$ & $20 \%$ & Earth aurora spectra driver \\
\hline Time sampling & $0.6 \mathrm{~s}$ & $0.5 \mathrm{~s}$ & $\leq 30 \mathrm{~km}$ auroral sampling/ \\
\hline Angle resolution & $30^{\circ}$ & $18^{\circ}$ using rotation & $\begin{array}{l}\text { Resolve loss cone for } \\
\mathrm{R}<2 / 3 \mathrm{RJ}\end{array}$ \\
\hline $\begin{array}{l}\text { Pitch Angle (PA) } \\
\text { Coverage }\end{array}$ & $\begin{array}{l}0-360 \text { degrees for whole } \\
\text { orbit }\end{array}$ & $\begin{array}{l}0-360 \text { degrees for whole } \\
\text { orbit }\end{array}$ & $\begin{array}{l}\text { Requires } 3 \text { JEDI heads } \\
\text { with } 160^{\circ} \times 12^{\circ} \text { fans }\end{array}$ \\
\hline $\begin{array}{l}\text { Time for Full PA } \\
\text { near Periapsis }\end{array}$ & $2 \mathrm{~s}$ & $1.25 \mathrm{~s}$ & $\begin{array}{l}\text { For high energy/angle } \\
\text { resolution }\end{array}$ \\
\hline Ion Composition & $\begin{array}{l}\mathrm{H} \text { and } \mathrm{S} / \mathrm{O} \text { over required } \\
\text { energies. } \\
\mathrm{He}: 70-1000 \mathrm{keV}\end{array}$ & $\begin{array}{l}\mathrm{H} \text { above } 15 \mathrm{keV} \\
\text { He above } 50 \mathrm{keV} \\
\mathrm{O} \text { above } 45 \mathrm{keV}\end{array}$ & $\begin{array}{l}\text { Separate } S \text { from } O \text { for } \\
E>200 \mathrm{keV}\end{array}$ \\
\hline $\begin{array}{l}\text { Electron } \\
\text { Sensitivity: } \\
\text { Measure energy } \\
\text { spectra }\end{array}$ & $\mathrm{I}=3 \mathrm{E} 5-3 \mathrm{E} 91 / \mathrm{cm}^{2} \mathrm{~s} \mathrm{sr}$ & $\begin{array}{l}\text { Sensor-G: } 0.0036-0.00018 \\
\text { Pixel-G: } 0.0006-0.00003 \\
\text { Up to } 5 E 51 / \text { s counting }\end{array}$ & $\begin{array}{l}\mathrm{I}=\text { Intensity }\left(1 / \mathrm{cm}^{2} \mathrm{sr}\right) \\
\mathrm{G}=\text { geom. factor } \times \text { eff. } \\
\left(\mathrm{cm}^{2} \mathrm{sr}\right) \\
\text { Variable } \mathrm{G} ; 6 \text { pixels/sensor }\end{array}$ \\
\hline $\begin{array}{l}\text { Ion Sensitivity } \\
\text { Measure energy } \\
\text { spectra }\end{array}$ & $\mathrm{I}=1 \mathrm{E} 4-1 \mathrm{E} 81 / \mathrm{cm}^{2} \mathrm{sr}$ & $\begin{array}{l}\text { Senso-G: } 0.002-0.0002 \\
\text { Pixel-G: } 0.0003-0.00003 \\
\text { Up to } 5 E 51 / \mathrm{s} \text { counting }\end{array}$ & $\begin{array}{l}\mathrm{I}=\text { Intensity }\left(1 / \mathrm{cm}^{2} \mathrm{sr}\right) \\
\mathrm{G}=\text { geom. factor } \times \text { eff. } \\
\left(\mathrm{cm}^{2} \mathrm{sr}\right) \\
\text { Variable } \mathrm{G} ; 6 \text { pixels/sensor }\end{array}$ \\
\hline
\end{tabular}

Again these requirements speak to both the JEDI instrument described in this paper, and the JADE instrument described elsewhere in this special issue.

\subsection{JEDI Level 3-4 Performance Requirements}

The Mission level requirements described above flow down to requirements for the payload (Level 3) and instruments (Level 4). At those levels the selected JEDI detailed performance requirements are provided in Table 1 . These requirements are generated based on previous measurements in Jupiter's space environment, extrapolations from measurements within Earth's polar regions, remote imaging of Jupiter's dramatic aurora from Hubble and Galileo, and from the characteristics of the Juno trajectory during the science phase at Jupiter. With regard to the requirements to separate mass species, we note specifically that we expect that JEDI will discriminate between Oxygen and Sulfur ions for energies $>200 \mathrm{keV}$ based on similar instruments. However, because it was discovered by Galileo (Mauk et al. 2004) that $\mathrm{S}$ and $\mathrm{O}$ spectra track each other very closely outside of the orbit of Europa and therefore on field lines that map to the target auroral regions, separating $\mathrm{S}$ from $\mathrm{O}$ is not a requirement for JEDI. An example of where Jupiter's polar characteristics and Juno mission characteristics meet is in the characterization of the smallest auroral structures that have been imaged at Jupiter ( $\sim 80 \mathrm{~km}$ wide; Ingersoll et al. 1998) and the speed of the Juno near Jupiter, up to $\sim 50 \mathrm{~km} / \mathrm{s}$. 


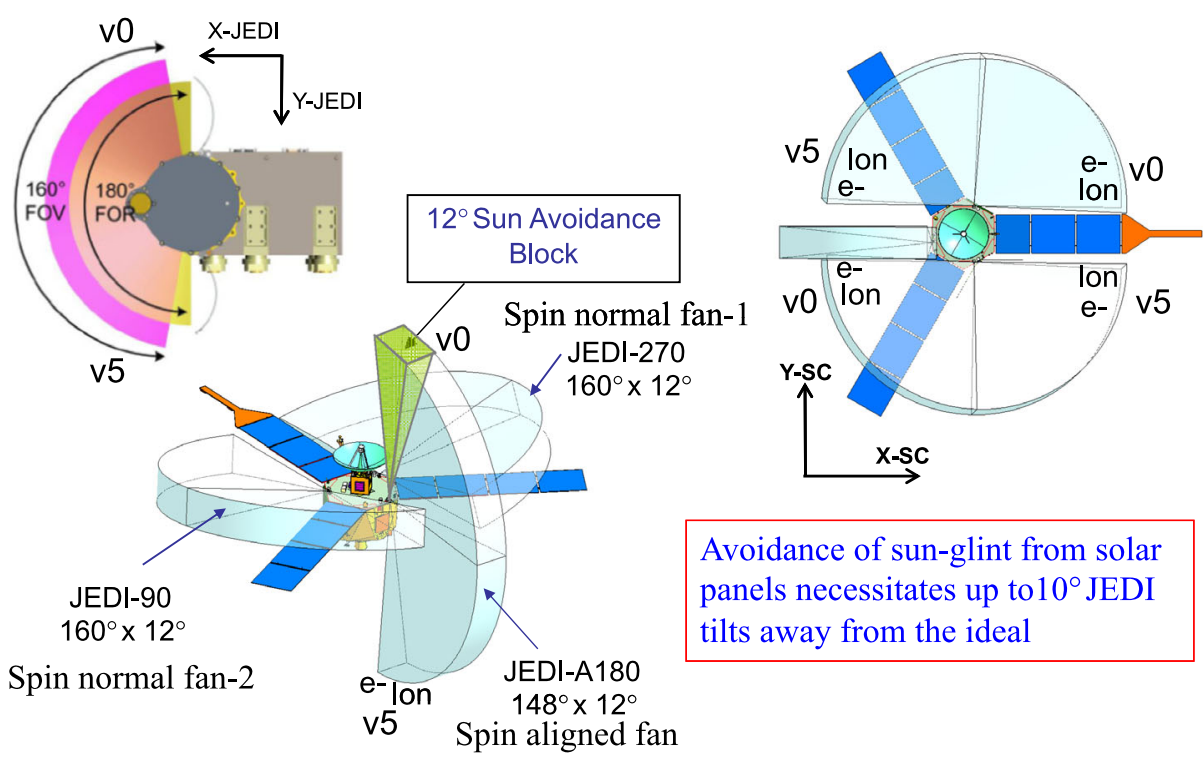

Fig. 2 Viewing configuration of the 3 JEDI sensors with respect to the Juno spacecraft. The JEDI coordinate system is shown in the upper left. The Juno spacecraft coordinate system is shown in the middle right. The JEDI view directions v0 and v5 are shown, along with the ordering of the electron and ion views (slightly rotated from each other) for some views

\section{JEDI Challenges}

There were substantial challenges to designing an energetic particle instrument that, given the characteristics of the Juno Mission, could deliver on the requirements listed in Sect. 2. Addressed here are four challenges: (1) A difficult viewing geometry given the slow spacecraft spin combined with the rapid spacecraft motions. (2) The huge dynamic range of input intensities expected for JEDI; (3) Penetrating radiation; and (4) The possible overwhelming fluxes of "out-of-band" low energy electrons and protons.

\subsection{Viewing Geometry}

The Juno spacecraft travels very rapidly in the close vicinity of Jupiter (up to $50 \mathrm{~km} / \mathrm{s}$ ) and also spins very slowly (2 RPM). Therefore, one may not take advantage of spacecraft spin to help sample different angles rapidly. What one must do is to view simultaneously into a multiplicity of directions. The approach that JEDI has taken is shown in Fig. 2. JEDI comprises 3 nearly-identical sensors, each of which already views simultaneously into 6 different directions over $160^{\circ}$ viewing fans. The 3 sensors are configured so that, given a nearly Earth-aligned spacecraft spin axis and a spacecraft orbit configuration that is initially roughly within a dawn-dusk plane, the 2 sensor heads that view roughly within the spacecraft equatorial plane instantaneously obtain nearly complete angle distributions with respect to the local magnetic field direction (pitch angle distributions); see Fig. 3. In the more distant regions of Jupiter's space environment, where the configuration changes much more slowly and where also the magnetic field is less ordered, the third head gives complete angular distributions every 30 second spin of the spacecraft (Fig. 3). An analysis of the viewing with respect to the magnetic field configuration close to Jupiter using the detailed orbit 
Fig. 3 Orientation of the JEDI Fields-of-View with respect to Jupiter's magnetic field close to Jupiter and early in the Juno science mission. Close to Jupiter nearly complete pitch angle distributions are measured at every instant of time $(0.5 \mathrm{~s}$ resolution)

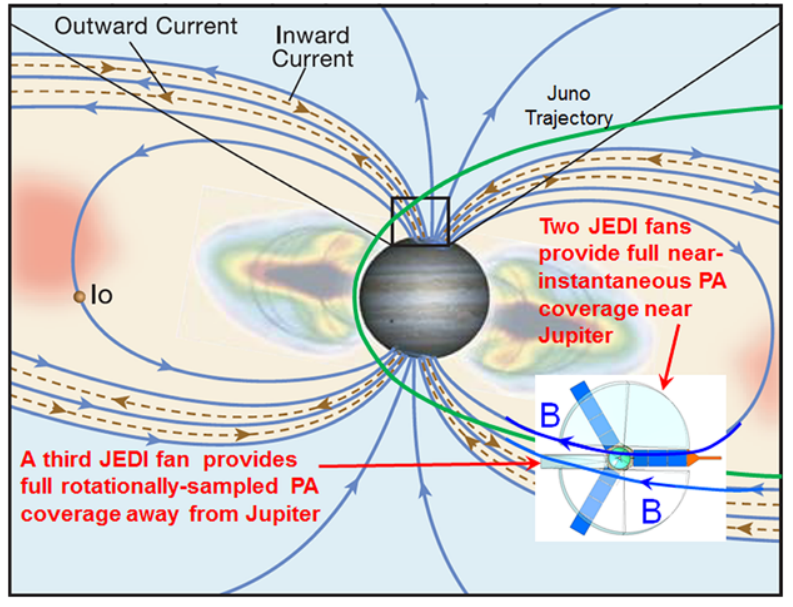

parameters and a prevailing detailed magnetic field model (O6; Connerney et al. 1992) is shown in Figs. 4 and 5. In these diagrams, complete angular viewing is achieved when the plotted parameter ( $y$-axis) is close to 90 degrees. We consider that JEDI is obtaining complete pitch angle distributions when that parameter is within the orange shaded region. The thick portion of the plotted lines are when the Juno spacecraft is within the prime auroral sampling region, that is when Juno resides on magnetic field lines that maps to the auroral regions, and when the Juno altitude is low enough such that JEDI can resolve the loss cone of the particle distributions. These analyses show that very good viewing of Jupiter's auroral processes will often be achieved with the choices that we have made for the JEDI design.

\subsection{Measurement Dynamic Range}

To characterize the various processes and regions that JEDI must measure within the Jupiter's space environment, JEDI must measure a very wide range of particle intensities. Peak intensities within Jupiter's dramatic aurora have brightness of several megaRayleighs (MR; 1 Rayleigh $=10^{6} / 4 \pi$ photons/[ $\left.\mathrm{cm}^{2} \mathrm{~s} \mathrm{sr}\right]$ ); see Fig. 6 (Elsner et al. 2005). Given that roughly $10 \%$ of precipitating electron energy flux is converted to auroral luminosity, and given the characteristic energy of the Lyman-alpha emissions $(\sim 13 \mathrm{eV})$, one may calculate the integral particle energy intensity needed to generate that flux under various assumed characteristic energies (in Table 2, a proposed maximum integral intensity that JEDI is capable of characterizing is the independent parameter on the left, and the different rows show the auroral luminosities that result from different assumed average energies of the JEDI-measured distributions). This calculation establishes the upper end of the integral intensities that must be measured by JEDI (Table 1). Examination of the intensities that must be measured elsewhere within Jupiter's space environment (Table 3) yields a requirement for JEDI to characterize 4 orders of magnitude of integral intensities separately for ions and electrons (Table 1) which expands to over 5 orders of magnitude given that the same sensor volume is used to measure both ions and electrons (sources for intensities within various regions include Mauk et al. 2004; Mauk and Fox 2010; Mauk and Saur 2007).

Two strategies are used to accommodate this very large dynamic range. First, the solid state detectors that measure the energy of the incoming particles are pixilated into large and small pixels, with a factor of 20 difference between them in area and in sensitivity. That 


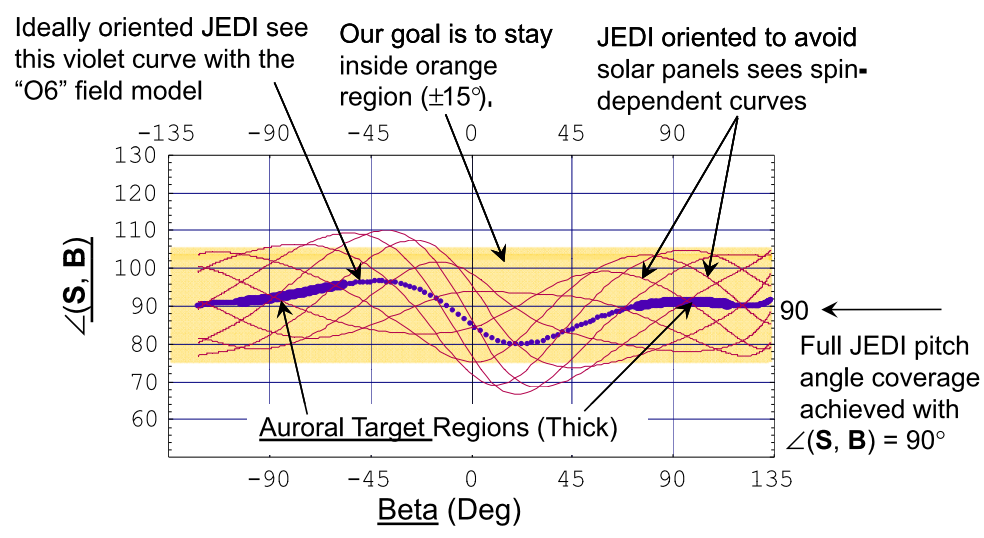

$\angle(\mathbf{S}, \mathbf{B})$ : Angle between magnetic field (B) and normal to JEDI view plane (S).

Beta: Jupiter-centered Juno orbit angle from equatorial crossing point.

Auroral Target: Offset Tilted Dipole invariant latitude $>70^{\circ}$ and Altitude $<2$ RJ

Fig. 4 Analysis showing how complete the pitch angle coverage of the JEDI sensors is close to Jupiter within the prime target region for Juno orbit \#3. The vertical axis is the angle between the normal to the JEDI view plane (established by JEDI-90 and JEDI-270) and the magnetic field. The ideal viewing is when the plotted lines reside closest to 90 degrees. The magnetic field is calculated on the basis of the so-called O6 magnetic field model (Connerney et al. 1992). The horizontal axis is the planetary latitude of the spacecraft-to-Jupiter line (connecting the center of Jupiter to the spacecraft). The spacecraft crosses the planetary equator near the center of the plot. For the plotted lines, the center of the cluster of the several lines represents the JEDI viewing if the JEDI fields-of-view plane was oriented exactly normal to the spacecraft spin axis. However, non-ideal tilts had to be introduced to keep the JEDI sensors from viewing the solar panels. The web of lines around the center line shows the dispersion of viewing with respect to the ideal case. The region where the center line is very thick is the auroral target region. Here the spacecraft is on magnetic field lines that are likely to map to the polar auroral regions and also the spacecraft is low enough in altitude so that the JEDI angular resolution capabilities can resolve the loss cone of the magnetic field. The horizontal orange region is the region within which we judge the JEDI field of view of obtaining complete pitch angle distributions

Table 2 JEDI Intensity versus Auroral Brightness capabilities

\begin{tabular}{|c|c|c|c|c|c|c|}
\hline \multicolumn{2}{|c|}{$\begin{array}{l}\text { Integral intensity }(>20 \mathrm{keV}) \\
1 /\left(\mathrm{cm}^{2} \mathrm{~s} \mathrm{sr}\right)\end{array}$} & \multirow[t]{2}{*}{$\begin{array}{l}\text { Average energy } \\
(\mathrm{keV})\end{array}$} & \multicolumn{2}{|c|}{$\begin{array}{l}\text { Energy flux } \\
\left(\mathrm{mW} / \mathrm{m}^{2}\right)\end{array}$} & \multicolumn{2}{|c|}{$\begin{array}{l}\text { Auroral brightness } \\
\text { (MR) }\end{array}$} \\
\hline Design & With margin & & Design & With margin & Design & With margin \\
\hline $5.00 \mathrm{E}+09$ & $1.00 \mathrm{E}+10$ & 30 & 754 & 1508 & 5.0 & 10.1 \\
\hline $5.00 \mathrm{E}+09$ & $1.00 \mathrm{E}+10$ & 100 & 2513 & 5027 & 16.8 & 33.5 \\
\hline $5.00 \mathrm{E}+09$ & $1.00 \mathrm{E}+10$ & 300 & 7540 & 15080 & 50.3 & 100.5 \\
\hline
\end{tabular}

factor of 20 is folded into the "count rates" shown in Table 3 for large pixels and small pixels. The second strategy used with JEDI for accommodating the very large dynamic range is to use very fast circuitry so that input rates as high as $5 \times 10^{5}$ counts/s per sensing element can be accommodated, with well-behaved responses as high as $10^{6}$ count/s (Sects. 4.3 and 5.3). This feature represents a substantial upgrade from the capabilities of the heritage instrument, the PEPSSI instrument on the New Horizons mission on its way to Pluto. 
Fig. 5 Similar to Fig. 4 but: (1) Four Juno orbits per panel are showing (Orbits 3 through 12 in all), (2) The webs of non-ideal viewing surrounding the central lines is not shown for clarity. The viewing configuration changes from orbit to orbit.
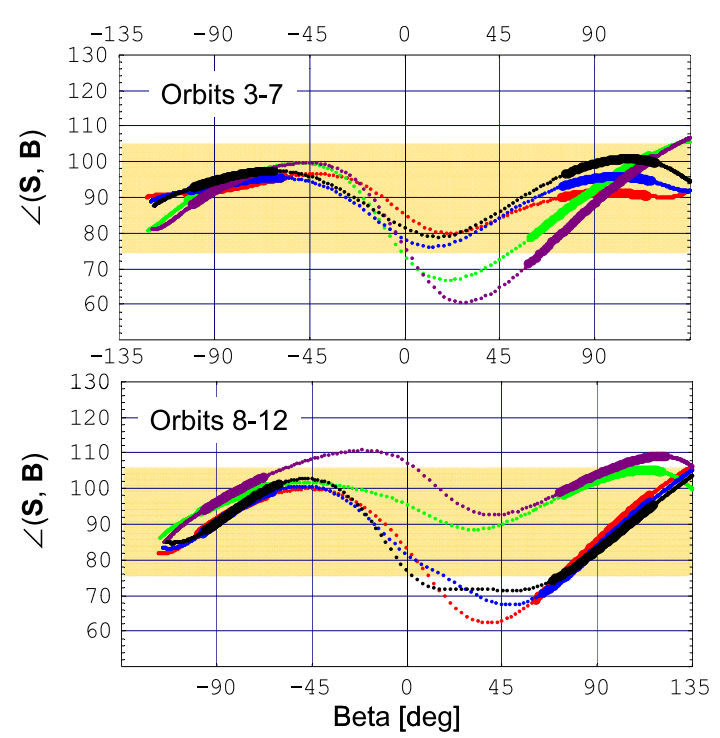

Fig. 6 Hubble image of Jupiter's aurora with color scale calibrated to emission intensity in MegaRayleighs (MR). After Elsner et al. (2005; figure version courtesy of R. Gladstone)

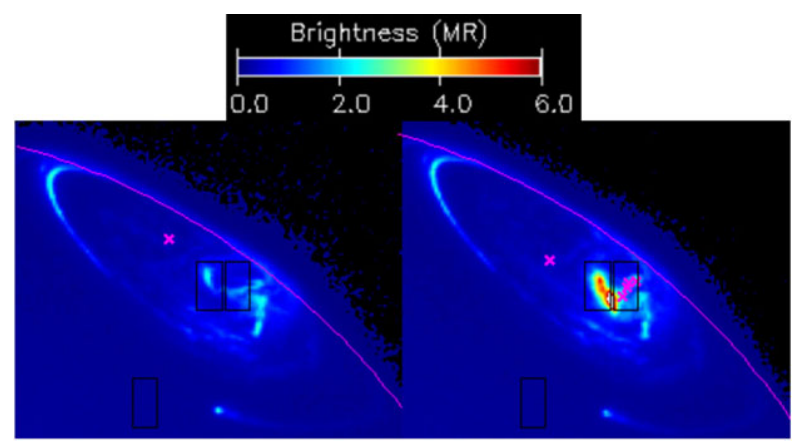

\subsection{Penetrating Radiation}

Jupiter's radiation electrons are unusually intense and energetic (Fig. 7; Garrett et al. 2003; Mauk and Fox 2010). These electrons provide a challenge for protecting sensitive electronics from radiation dose damage, and also a challenge in guarding against contaminating background in the solid-state detector (SSD) and microchannel-plate (MCP) sensors. The former problem is mitigated on JEDI with thick shielding $(0.25 \mathrm{~cm}$ of a Tungsten-Copper mixture with density of order $>15 \mathrm{~g} / \mathrm{cm}^{3}$ ) yielding an estimated mission dose of about $25 \mathrm{krads}$. JEDI uses combinations of hard electronics parts and spot shielding to yield a tolerance of up to $100 \mathrm{krads}$. The consequence to this mitigation is that each of the 3 sensors, which nominally would have mass in the $\sim 2 \mathrm{~kg}$ range, instead had mass, including shielding, of roughly $\sim 6.4 \mathrm{~kg}$.

The backgrounds in measurement caused by the penetrating electrons can be significant, and there are certainly regions of the magnetosphere where JEDI will not make quality measurements. And so, where is JEDI required to make quality measurements? The prime target of the suite of space environment instruments is Jupiter's auroral region. The main auroral oval is known to map magnetically to regions in the vicinity of and beyond the orbit 
Table 3 Expected count rates requiring very large dynamic range for JEDI

\begin{tabular}{|c|c|c|c|c|}
\hline $\begin{array}{l}\text { Position/conditions } \\
\text { species }\end{array}$ & $\begin{array}{l}\text { Integ. intensity } \\
1 /\left(\mathrm{cm}^{2} \mathrm{~s} \mathrm{sr}\right) \\
(>20 \mathrm{keV})\end{array}$ & $\begin{array}{l}\text { Valid TOF } \\
\text { Start rate } \\
(1 / \mathrm{s})\end{array}$ & $\begin{array}{l}\text { Large SSD rate } \\
\text { Small SSD rate } \\
(1 / \mathrm{s})\end{array}$ & Comment \\
\hline $\begin{array}{l}\text { Ions Galileo } \mathrm{R}=40 \\
\text { Aurora outer limit }\end{array}$ & $1 \mathrm{E} 5$ & $\begin{array}{l}300 \\
1800\end{array}$ & $\begin{array}{l}100 \\
5\end{array}$ & $\begin{array}{l}10 \times \text { Low Ion Design } \\
\text { Limit }\end{array}$ \\
\hline $\begin{array}{l}\text { Ions Galileo } \mathrm{R}=9.5 \\
\text { Europa }\end{array}$ & $6 \mathrm{E} 6$ & $\begin{array}{l}1.8 \mathrm{E} 4 \\
1.1 \mathrm{E} 5\end{array}$ & $\begin{array}{l}6000 \\
300\end{array}$ & Ions \\
\hline $\begin{array}{l}\text { Guess Auroral Peak } \\
30 \text { times Europa }\end{array}$ & $2 \mathrm{E} 8$ & $\begin{array}{l}6 \mathrm{E} 5 \\
3.6 \mathrm{E} 6\end{array}$ & $\begin{array}{l}2 \mathrm{E} 5 \\
1 \mathrm{E} 4\end{array}$ & High Ion Design Limit \\
\hline $\begin{array}{l}\text { Cravens X-ray Ion } \\
\text { Flare } \\
\text { A GOAL }\end{array}$ & $\begin{array}{l}\text { Flux: } 2 \mathrm{E} 7 \\
\left(1 / \mathrm{cm}^{2} \mathrm{~s}\right) \\
\text { Mono-directional }\end{array}$ & $\begin{array}{l}1 \mathrm{E} 6(50 \% \text { of } 6 \\
\text { holes) } \\
1 \mathrm{E} 6 \text { (the same) }\end{array}$ & $\begin{array}{l}8 \mathrm{E} 6 \\
4 \mathrm{E} 5\end{array}$ & Rare/Unlikely Ion Event \\
\hline $\begin{array}{l}\text { Electron Galileo } 40 \\
\text { RJ }\left(0.3 \text { ergs } / \mathrm{cm}^{2} \mathrm{~s}\right)\end{array}$ & $5 \mathrm{E} 5$ & N/A & $\begin{array}{l}500 \\
25\end{array}$ & $\begin{array}{l}10 \times \text { Low Electron } \\
\text { Design Limit }[\#]\end{array}$ \\
\hline $\begin{array}{l}\text { Voyager @ 14-16 RJ } \\
\text { Elect. }\left(30 \text { ergs } / \mathrm{cm}^{2} \mathrm{~s}\right)\end{array}$ & $5 \mathrm{E} 7$ & N/A & $\begin{array}{l}5 \mathrm{E} 4 \\
2500\end{array}$ & Electrons \\
\hline $\begin{array}{l}\text { Arnoldy Peak ( } 750 \\
\left.\mathrm{ergs} / \mathrm{cm}^{2} \mathrm{~s}\right) @ 30 \mathrm{keV}\end{array}$ & $5 \mathrm{E} 9$ & N/A & $\begin{array}{l}5 \mathrm{E} 6 \\
2.5 \mathrm{E} 5\end{array}$ & $\begin{array}{l}\text { High Electron Design } \\
\text { Limit }\end{array}$ \\
\hline
\end{tabular}

Fig. 7 Electron radiation spectra for the equatorial radial ranges at Jupiter from 8.25 RJ to $16.25 \mathrm{RJ}$. The spectral parameters are from Garrett et al. (2003) and the plot of those parameters is from Mauk and Fox (2010)

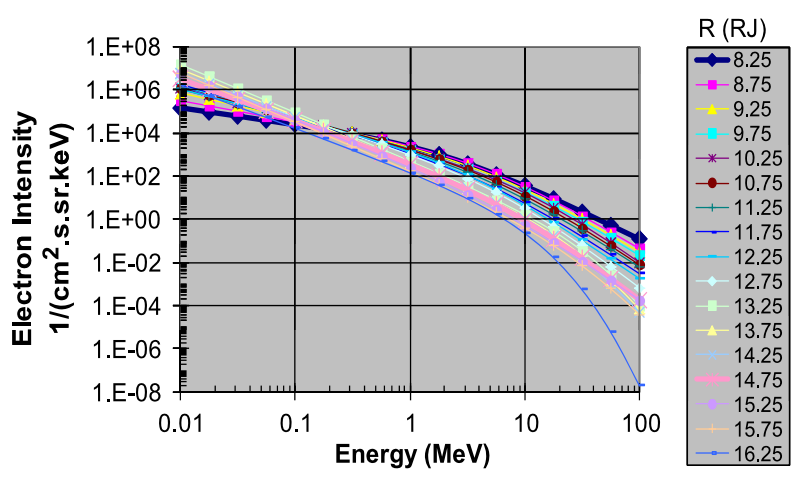

of Ganymede, with an orbital radial position of about 15 RJ (Clarke et al. 2002). If we assume as a worst case that the radiation spectrum at low altitudes on magnetic field lines that map to Ganymede's orbit is as intense as it is in the equatorial regions, then we can say that JEDI must take quality measurements in a radiation environment that is as intense as the shown in Fig. 7 for radial positions equal to $14.75 \mathrm{RJ}$ or higher. It is our goal to also make quality measurements for radiation environments as intense as those seen in the orbit or Europa, at a radial distance of about 9.5 RJ (e.g. the 9.75 RJ in Fig. 7).

The Energetic Particle Detector (EPD) on the Galileo mission to Jupiter (Williams et al. 1992) had a Microchannel Plate (MCP) sensor similar to that of JEDI, and it was shielded with metal shielding with a mass thickness of about $5 \mathrm{~g} / \mathrm{cm}^{2}$. By measuring the MCP singles rates near Europa, near Ganymede, and in various places in between at a time when the field-of-view was positioned behind a $2 \mathrm{~mm} \mathrm{Al}$ foreground shield, and by extrapolating those rates for other shielding thicknesses using the spectral shapes of the electrons spectra (Fig. 7), we have performed a parametric study of the MCP background rates that JEDI is likely to see in the environments of both Ganymede and Europa (Table 4). Because the 
Table 4 Shielding required to protect the JEDI MCP's

Note 1: Scaling from Galileo EPD MCP singles rates

Note 2: JEDI tolerates $10^{4} \mathrm{MCP}$ $\mathrm{BG}$ rates (coincident circuitry)

Note 3: Includes all secondary products (e.g. X-rays)

Note 4: Factors of 2 uncertainty should be assumed

\begin{tabular}{|c|c|c|c|}
\hline & $\begin{array}{l}\text { Shield } \\
\text { thickness } 1\end{array}$ & $\begin{array}{l}\text { Shield } \\
\text { thickness } 2\end{array}$ & $\begin{array}{l}\text { Shield } \\
\text { thickness } 3\end{array}$ \\
\hline Shielding $\left(\mathrm{gm} / \mathrm{cm}^{2}\right)$ & 2.9 & 5.2 & 9.3 \\
\hline Penetrating energy $(\mathrm{MeV})$ & 5.6 & 10 & 17.8 \\
\hline W-Cu thickness (mils) & 69 & 123 & 220 \\
\hline $\begin{array}{l}\text { Ganymede total MCP rate } \\
\text { (cnts/s) }\end{array}$ & $4.8 \mathrm{E} 4$ & $1.4 \mathrm{E} 4$ & $3.5 \mathrm{E} 3$ \\
\hline $\begin{array}{l}\text { Ganymede MCP rate per } \\
\mathrm{cm}^{2}(\mathrm{cnts} / \mathrm{s})\end{array}$ & $2.4 \mathrm{E} 3$ & 710 & 180 \\
\hline $\begin{array}{l}\text { Europa total MCP rate } \\
\text { (cnts/s) }\end{array}$ & $8.4 \mathrm{E} 5$ & $3.6 \mathrm{E} 5$ & $1.3 \mathrm{E} 5$ \\
\hline $\begin{array}{l}\text { Europa MCP rate per } \mathrm{cm}^{2} \\
\text { (cnts/s) }\end{array}$ & $4.3 \mathrm{E} 4$ & $1.8 \mathrm{E} 4$ & 6700 \\
\hline
\end{tabular}

MCP foreground events are used only with coincident circuitry, the JEDI measurements that depend on the MCP measurements can tolerate substantial background counts. Background rates of $10^{4}$ counts per second are essentially invisible to JEDI from the perspective of JEDI measurements (the time window for the coincident measurements is no longer than $160 \mathrm{~ns}$ ). So-called "accidental" rates (background rates that look like foreground events) start becoming significant as the MCP background rates rise above $10^{5}$ counts per second. Table 4 shows that, with the shielding that the JEDI MCP has, mostly $7.5-8 \mathrm{~g} / \mathrm{cm}^{2}$ (some directions as low as $5 \mathrm{gm} / \mathrm{cm}^{2}$; Appendix A), the MCP measurements allow for very high quality measurements in the Ganymede environment, and somewhat degraded, but still highly useful, measurements in the vicinity of Europa. Signal processing mitigations (e.g. demanding that the directional sector that the particle enters matches the directional sector in the back end of the sensor volume) allows us to beat down the MCP contamination events by a factor of greater than 3 from those shown in the table. The MCP rates shown in Table 4 are also conservative because one expects that the spectra at low altitudes to be significantly reduced from those in Fig. 7 because of the magnetic mirror trapping and scattering losses for particles that mirror close to the atmosphere.

The other factor of concern for penetrating radiation is the contamination of the measurements by the Solid State Detectors. The geometric factor for the detection of the very high energy electrons that penetrate the shielding is much larger than the geometric factor for the measurement of the lower energy electrons that comprise the foreground measurement of electrons (the measurement of ions is not very much contaminated by SSD events because of the triple-signal double coincident circuitry used to measure the ions (start-stopSSD measurements). The electrons are detected using non-coincident singles measurements within SSD's and so contamination by the penetrating electrons is a significant concern. And, the problem is most serious when the electron spectrum is very hard, i.e. for an intensity spectral shape of $E^{-\gamma}$ where $E$ is energy and $\gamma$ is the spectral index, a spectrally hard spectrum means that $\gamma$ is a low number like 1 or 2 .

Our mitigation for penetrating radiation effects on the SSD's is two-fold: (1) use as much shielding as we can given resource limitations, and (2) make use of what we call "witness" detectors to measure the spectra of contamination directly. To the best of our ability, we have shielded the SSD with a thickness of Tungsten-Copper mixture of $0.5 \mathrm{~cm}$ for most directions, but as low as $0.3 \mathrm{~cm}$ for some limited directions (Appendix A). The $0.5 \mathrm{~cm}$ thickness corresponds to about $7.5-8 \mathrm{~g} / \mathrm{cm}^{2}$ of shielding, which shields most electrons with energies up to approximately $>15 \mathrm{MeV}$. Figure 8 shows what we mean by "witness" detectors. As 

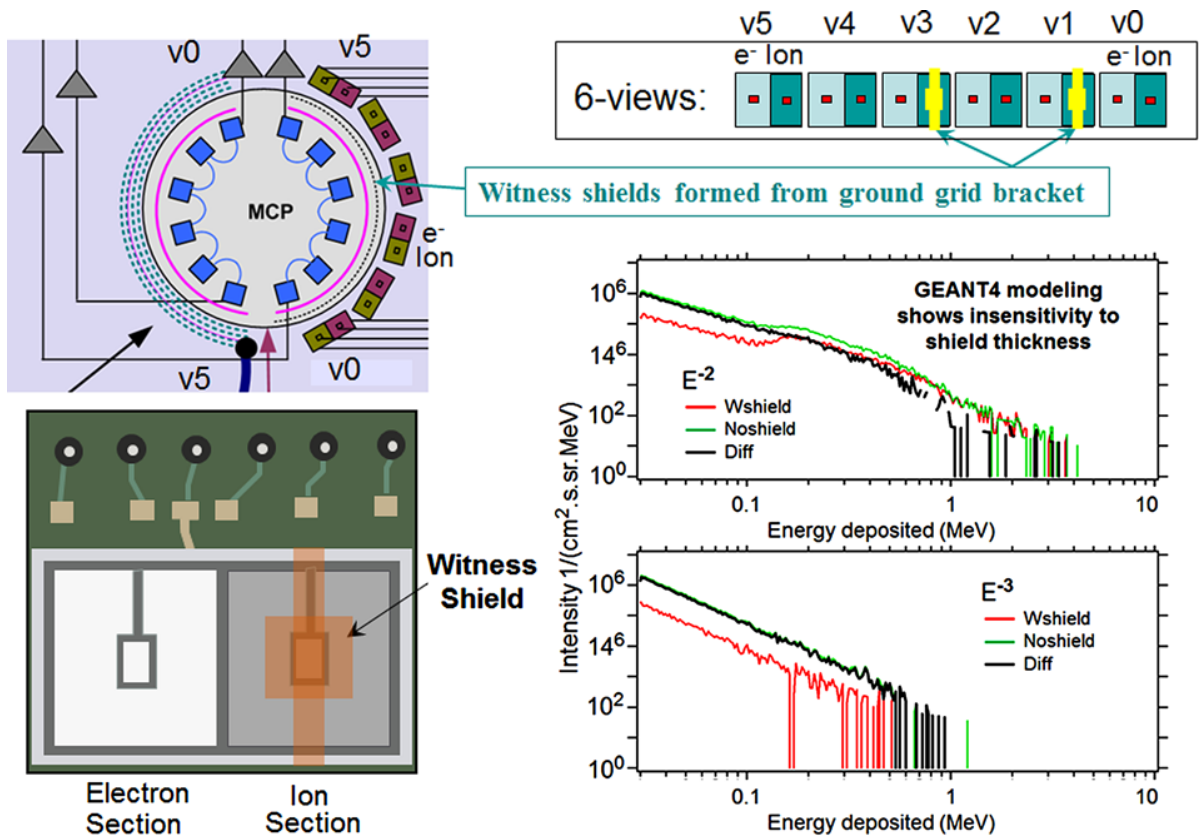

Witness shields formed from ground grid bracket

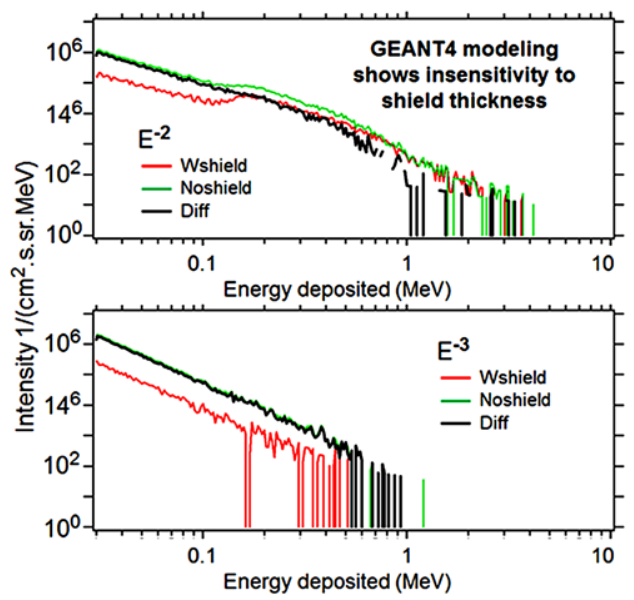

Fig. 8 Exhibits documenting the "witness detectors" on the JEDI-A180 instrument. Across two out of 6 of the ion SSD small pixels (v1 and v3 out of the $6 \mathrm{v0}-\mathrm{v} 5$ views) is a $\sim 2 \mathrm{~mm} \mathrm{Al} \mathrm{equivalent} \mathrm{absorber.}$ The GEANT4 analysis shown in the lower right shows how subtraction of the witness detector spectrum from the spectra taken from the unshielded directions can reconstruct the primary spectra (subtracting off the contributions from high energy electron penetrators. This function is needed for very hard spectra with high energy tails that extend to energies $>10 \mathrm{MeV}$

will be explained more fully in Sect. 4, the JEDI SSD's for each direction are pixilated with "large" pixels" and "small" pixels. For just one of the 3 JEDI heads (designated JEDIA180), two of the small ion pixels (for "views" 1 and 3, designated v1 and v3 out of the 6 views: v0-v5) have $\sim 2 \mathrm{~mm} \mathrm{Al} \mathrm{equivalent} \mathrm{shields} \mathrm{over} \mathrm{them,} \mathrm{which} \mathrm{removes} \mathrm{the}<1$ $\mathrm{MeV}$ foreground electrons from reaching these pixels, allowing these pixels to just measure the penetrating background (upper right and lower left of Fig. 8). But, because these pixels see the same penetrating environment as the unshielded adjacent pixels, the outputs of the shielded and unshielded pixels can be compared directly. The lower right of Fig. 8 shows a simulation using the GEANT4 software whereby the green curve is the measurement of an unshielded pixel, the red curve shows the measurement of the shielded pixel, and the black curve is the difference, which essentially restores the input spectra. A comparison between the top panel and the bottom panel shows that this "correction" is only needed for spectrally hard spectra ( $\gamma=2$ rather than the softer $\gamma=3$; to be a problem the spectrum must extent in energy to $>10 \mathrm{MeV}$ with the same hard spectral index, as modeled in Fig. 8)

\subsection{Electron and Low Energy Plasma Contamination}

In early time-of-flight by energy (TOF $\times$ E) ion spectrometers (AMPTE CCE, Galileo EPD, Geotail EPIC), the front "start" foil was protected from electrons by carefully tailored magnetic fields using permanent magnets. As will be described in Sect. 4, the start foil is a very thin film that is penetrated by the incoming ion and, as a result, emits secondary electrons 
Fig. 9 Energy-dependent efficiency for the generation of secondary electrons from the exit surface of thin carbon foils resulting from the penetration of electrons. Such electron interactions with the start and stop foils of JEDI are a contaminant to the measurement of the properties of ions and must be considered in the design of the instrument. Extrapolated Hölzl and Jacobi (1969) measurements, are used here for evaluating possible contaminations of the JEDI measurements. Funsten et al. (2012) validate this approach

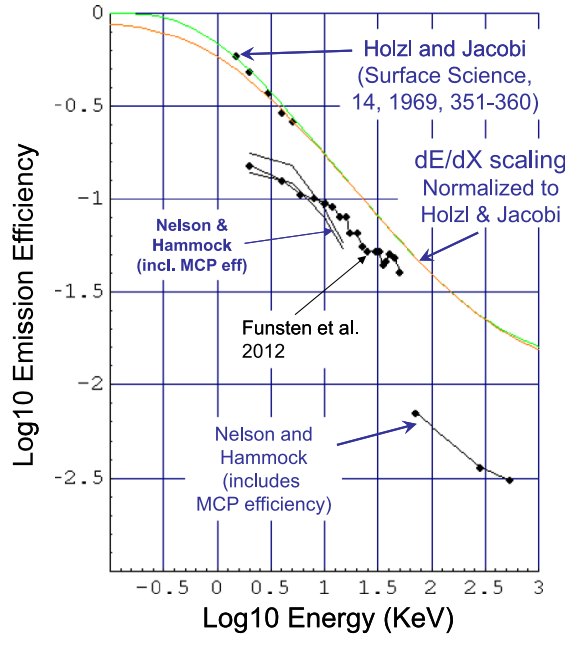

Fig. 10 In order to suppress the contribution of out-of-band low energy electrons and ions to the response of the JEDI foils, a 3rd foil, the "collimator foil," was added to the collimator of JEDI. The right panel shows a GEANT4 evaluation of that suppression as a function of energy for various species
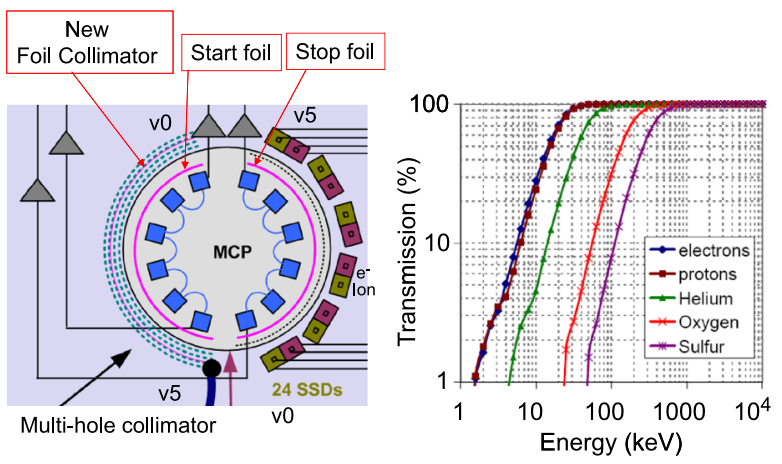

that are deflected and detected by the MCP. A magnetic field can protect the start foil from the generation of secondary electrons by incoming energetic electrons. On New Horizon's PEPSSI the same sensor is used to measure both ions and electrons, and so no magnetic field was included. However, the PEPSSI TOF $\times$ E sensor is designed to measure ions in the interplanetary environment close to the (assumed to be) un-magnetized planet Pluto. Generally the electron intensities do not compete with the ion intensities, and during the years of cruise of the PEPSSI instrument on its way to Pluto, the ion measurements have not been generally contaminated with large electron intensities that stimulate the front foil. However, within an active, strongly magnetized magnetosphere like that of Earth and Jupiter, the electron intensities can be very intense and have the potential of disrupting the measurement of the ions by overwhelming the start pulse processing circuitry.

Several studies of the efficiency of secondary electron generation of electrons penetrating thin carbon foils are shown in Fig. 9. That efficiency is unfortunately very high for electrons with energies up to several keV. For our design efforts we have adopted the Hölzl and Jacobi (1969) results and have extrapolated those data with the assumption that the secondary electron emission efficiency depends on the $d E / d X$ curve of electrons within carbon. Modeling this curve with known electron distributions within Earth's and Jupiter's magnetospheres in regions where we need to make ion measurements, we find that indeed the electron input can sometimes substantially contaminate the ion measurements. 
Our mitigation for this contamination is shown in Fig. 10. As will be discussed in Sect. 4, the JEDI collimator comprises 5 cylindrically shaped blades with multiple aligned holes in them through which the particles pass (left panel of Fig. 10). What we have done is added a 3rd thin foil, made of $350 \AA$ of aluminum (specifically we took some of the mass that otherwise would reside within the start foil and stop foil to suppress visible and ultraviolet light and put it into this third foil). Low energy particles scatter within this foil and are fractionally stopped by the inner two blades of the collimator. The right hand panel of Fig. 10 shows that the lower energy particles, most importantly the electrons about which we have been speaking, are substantially suppressed. That suppression is sufficient to yield clean ion measurements within the regions where such clean measurements are needed. We, of course, have also compromised JEDI's sensitivity to the lower energy range of its ion measurements, but it was a compromise that we needed to make.

\section{The JEDI Instrument}

Here we describe in some detail the design, hardware and inner workings of the JEDI instrument suite. That suite comprises three nearly identical instruments, two mounted in a horizontal configuration and one mounted in a vertical configuration (relative to the spacecraft deck; Fig. 2). Each instrument comprises two subsystems, the sensor head and the main electronics (Fig. 11, upper right). Each sensor head incorporates electron and ion sensors (e.g. Fig. 10 and later discussions), plus detector preamplifiers. The sensor head and main electronics are mechanically integrated together and mounted as a single unit to the spacecraft. Each JEDI instrument has a separate power and data interface to the spacecraft, and therefore each appears as separate "instrument interface". The instruments run independently of each other; there is no intra-suite communication between the three instruments.

Note that to keep the technical descriptions simple and straight forward, we do not provide many of the technical specifications for the JEDI instrument in this section (mass, power, sizes, materials, densities, thicknesses, gaps, voltages, etc.). Those specifications are provided in Appendix A.

\subsection{Principles of Operation}

JEDI measures ion energy, directional, and compositional distributions using Time-of-Flight by Energy $(\mathrm{TOF} \times \mathrm{E})$ and Time-of-Flight by MCP-Pulse-Height $(\mathrm{TOF} \times \mathrm{PH})$ techniques. JEDI measures electron energy and directional distributions using collimated solid-statedetector (SSD) energy measurements (these electron SSD's, as opposed to the ion SSD's, have 2 microns of aluminum flashing deposited on them to keep out protons with energies less than about $250 \mathrm{keV}$ ). JEDI combines multidirectional viewing into individual compact sensor heads (Fig. 11). The sensor heads include time-of-flight (TOF) sections about $6 \mathrm{~cm}$ across feeding a solid-state silicon detector (SSD) array. The SSD array and its associated preamps are connected to an "event board" (next section) that determines particle energy. Secondary electrons, generated by ions passing through the entry and exit foils (Fig. 11 left), are detected by the microchannel plate (MCP) stimulated timing anodes and their associated preamps to measure ion TOF. Event energy $(E)$ and TOF measurements are combined to derive ion mass and to identify particle species.

The JEDI acceptance angle is fan-like and measures $160^{\circ}$ by $12^{\circ}$ with six $\sim 26.7^{\circ}$ look directions. Particle direction is determined by the particular look direction in which it is de- 

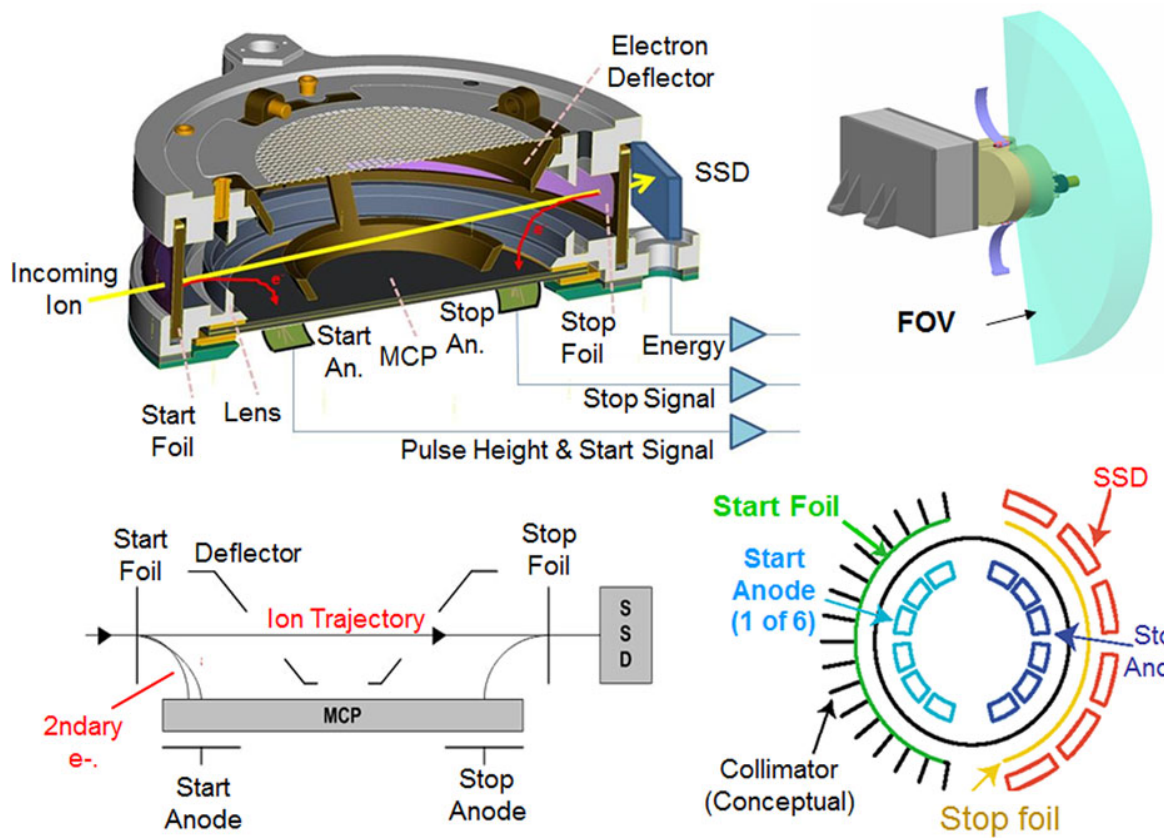

Fig. 11 Exhibits showing how each JEDI sensor operates

tected (six different view directions for each species, label v0, v1, v2, v3, v4 and v5). That directionality is determined by the active SSD in the case of electrons, and by the determination of the entrance position on the MCP-stimulated time-delay anode nearest to the start foil in the case of ions (time delay along a chain of 12 "start" anode pads connected by inductors is used to determine entrance position). Ions that pass through the sensor encounter three separate thin foils mounted on $\sim 90 \%$ transmission grids. The first one, the "collimator foil," mounted within the collimator, is a $350 \AA$ aluminum foil. The next foil, the

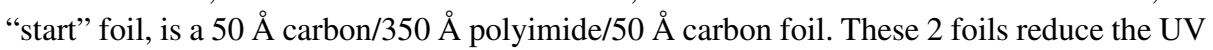
(e.g. Lyman alpha) photon background. The exit apertures are covered by the third or "stop" foil of $50 \AA$ carbon/350 ̊ polyimide/50 A carbon/ $200 \AA$ aluminum. All foils are mounted a high-transmittance $(90 \%)$ metal grids supported on stainless steel frames (START and STOP foils) or tungsten copper frame (COLLIMATOR foil).

\subsubsection{Electron Sensors}

Before an electron passes through the TOF head, it is first decelerated by a $2.6-\mathrm{kV}$ potential (part of the TOF optics for measuring ions); it is later reaccelerated by $2.6-\mathrm{kV}$ after exiting the stop foil prior to reaching the SSD detectors. Energetic electrons from $25 \mathrm{keV}$ to $1000 \mathrm{keV}$ are measured by the electron SSD detectors. The electron detectors are covered with $2-\mu \mathrm{m}$ aluminum metal flashing to keep out protons and ion particles with energies less than about $250 \mathrm{keV}$. No TOF criterion is applied to the electron measurements. The sensitivity to particles can be adjusted by a factor of 20 by selecting large or small SSD pixels (discussed below). 


\subsubsection{Ion Sensors}

Before an ion passes through the TOF head, it first passes through a thin foil in the collimator (350 A aluminum). It then penetrates first the start foil and then the stop foil (Fig. 11 left). Secondary electrons from the start and stop foils are electrostatically separated from the primary particle path and diverted on the microchannel plate (MCP), providing start and stop signals for TOF measurements. The segmented MCP anodes, with two start and two stop anodes for each of the six angular segments, determine the direction of travel. A 500volt accelerating potential between the foil and the MCP surface controls the electrostatic steering of secondary electrons. The dispersion in electron transit time is less than $1 \mathrm{~ns}$. As an aside we should note that after penetrating a foil, the ion may emerge as an ion or as a neutral. If it emerges as an ion from the collimator foil it is accelerated by a negative $2.6 \mathrm{kV}$ potential on the TOF Start foil; after passing through the Start and the Stop foil, it again may have changed charge state. Assuming it remains an ion, it is then decelerated by $2.6 \mathrm{kV}$ after exiting the head prior to reaching the SSD detectors. Below $30 \mathrm{keV}$, a proton has less than a $50 \%$ chance of remaining charged on exiting a foil. At $10 \mathrm{keV}$ the probability drops to $20 \%$.

Ion energy measurements using the ion detectors are combined with coincident TOF measurements to derive particle mass and identify particle species (the TOF $\times$ E method). With the TOF $\times$ E method the incoming particles are measured from $50 \mathrm{keV}$ to above $1 \mathrm{MeV}$; they are discriminated in the energy system above $50 \mathrm{keV}$ for protons and above $150 \mathrm{keV}$ for heavy ions (such as the CNO group). An example of a TOF $\times \mathrm{E}$ matrix and how it separates different mass species is shown in the lower left panel of Fig. 12B from the New Horizons PEPSSI instrument at Jupiter (McNutt et al. 2008). Lower-energy ion fluxes are measured using TOF-only measurements (the TOF $\times$ Pulse Height method); detection of MCP pulse height provides a coarse indication of low-energy particle mass. An example of how a TOF $\times$ PH spectrum crudely separates different ion mass species at Earth, from the IMAGE HENA instrument, is shown in Fig. 12D (Mitchell et al. 2003). Sensitivity to higher energy ions (those with energies above the SSD channel thresholds) can be adjusted by selecting large or small SSD pixels.

\subsection{Heritage}

The Johns Hopkins APL has generated and flown numerous TOF $\times$ E instruments, generally including SSD-based electron sensors, on numerous spacecraft. The list includes the Earth-orbiting AMPTE CCE MEPA instrument (McEntire et al. 1985) and Geotail EPIC instrument (Williams et al. 1994), the Jupiter-orbiting Galileo EPD instrument (Williams et al. 1992), and the New Horizon's PEPSSI instrument now on its way to Pluto (McNutt et al. 2008). Instruments that have used the TOF $\times$ PH method include the Earth-orbiting IMAGE HENA instrument (Mitchell et al. 2003) and the Saturn-orbiting Cassini MIMI INCA instrument (Krimigis et al. 2004). The instrument that is closest to the JEDI instrument is the New Horizons PEPSSI instrument. That instrument is pictured in Fig. 12A, along with data taken during the New Horizons encounter with Jupiter, into a radial position of $39 \mathrm{RJ}$ (Figs. 12B and 12C). At its inner most position of 39 RJ, PEPSSI cleanly separated electrons and ions and delivered quality ion composition data. The first real scientific use of the TOF $\times$ PH technique began with IMAGE HENA instrument (data shown in Fig. 12D), and the mass discrimination on the Cassini MIMI INCA instrument at Saturn is based solely on that technique. Finally, a sister instrument to JEDI, RBSPICE on the Van Allen Probes mission, has now been launched (Mitchell et al. 2013). 


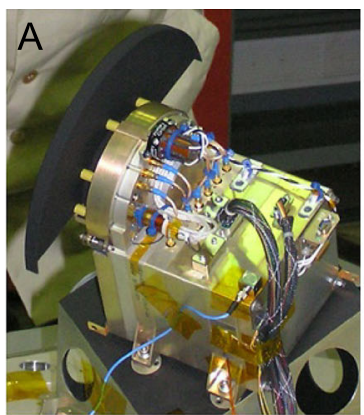

Log10 Energy (keV)

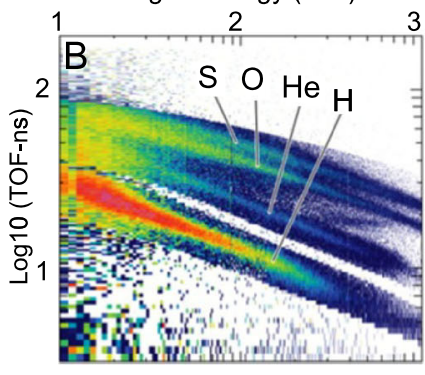

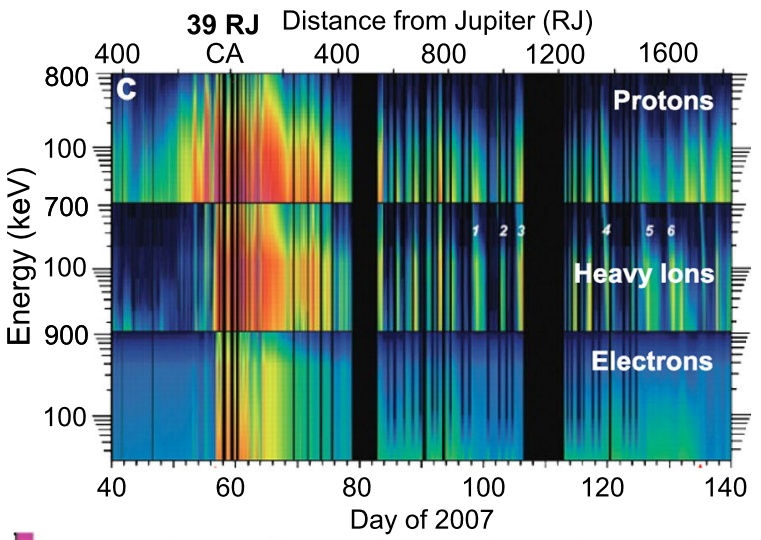

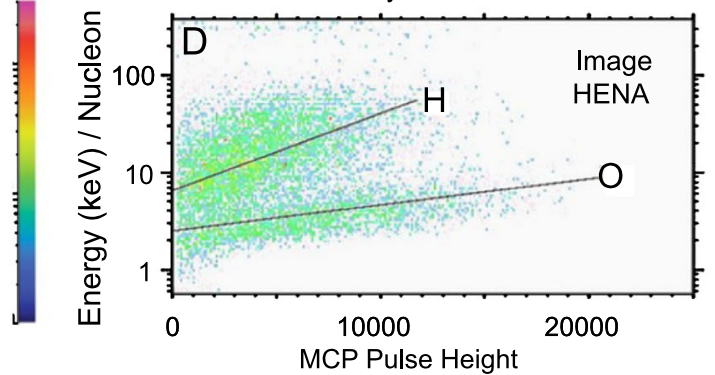

Fig. 12 The most recent heritage instrument to JEDI, the New Horizons PEPSSI instrument along with several measurements made by PEPSSI during the New Horizons encounter with Jupiter (upper right and lower left). The lower right shows TOF $\times$ Pulse-Height measurements made at Earth from the IMAGE HENA instrument

\subsection{JEDI Block Diagram and Details of the Electronic Design}

The JEDI instrument block diagram is shown in Fig. 13. Note that the hardware and interfaces shown in this diagram apply to each of the three JEDI sensor heads.

On the left, the sensor generates analog representations of particle Time-of-Flight (TOF) and energy from the SSD's. Each SSD has both electron and ion pixels. There is only one analog electronics processing chain per SSD. Consequently, to collect both electrons and ions, the hardware must be time-multiplexed between the electron and ion detectors. Similarly, the event processing logic is switched between modes that measure ion energy vs. ion species. The hardware is time-multiplexed between three possible modes: electron energy, ion energy, and ion species. An event trigger selects what combination of TOF and SSD pulses defines an event. With energy trigger, an SSD energy $(E)$ pulse defines an event. With TOF trigger, a TOF pulse, with or without an E pulse defines an event. Time-multiplexing of the hardware is done by the software, which is required to measure only electrons or only ions at any one time. So, many commands have options for specifying electron or ion settings. For example, the command that controls energy discriminator thresholds specifies either electron or ion discriminator settings. The software time-multiplexes the actual discriminator threshold between the electron and ion settings. For JEDI, it will be typical to cycle through the typically 2 or sometimes 3 different species modes every $0.5 \mathrm{~s}$ (see later discussion). 


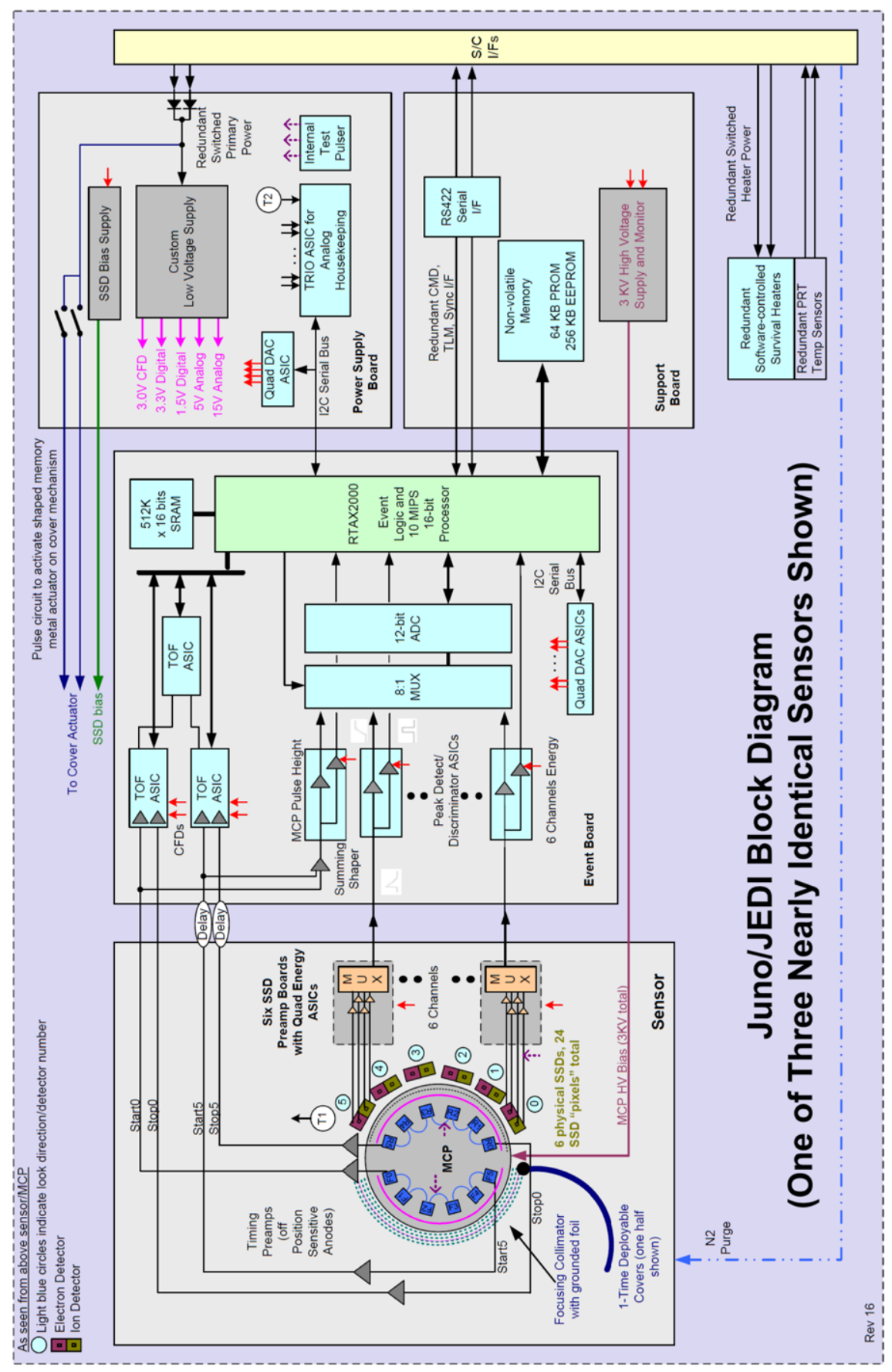

Fig. 13 The JEDI block diagram showing how functionality is distributed and showing the electrical interfaces with the Juno spacecraft 
Table 5 Particle Event Data

\begin{tabular}{lll}
\hline Name & Bits & Description \\
\hline TOF1 & 10 & TOF chip 1, Stop0-Start0 \\
TOF2 & 10 & TOF chip 2, Stop5-Start5 \\
TOF3 & 10 & TOF chip 3, Start5-Start0 \\
TOF Flags & 3 & TOF chip validity flags (VE3, VE2, VE1) \\
TOF & 10 & Corrected TOF, average of TOF1 and TOF2 \\
Start Chan & 3 & Calculated start channel, 0-5 (6, 7 = invalid) \\
Stop Chan & 3 & Calculated stop channel, 0-5 (6, 7 = invalid) \\
MCP PH & 12 & MCP pulse height \\
MCP PH Flag & 1 & MCP PH flag (0= no pulse, 1 = pulse) \\
SSD Energy & 12 & SSD energy \\
SSD Coin Flags & 2 & SSD with start; SSD with stop \\
SSD Flags & 6 & SSD flags $(0=$ no pulse, 1 = pulse) \\
SSD Chan & 3 & SSD channel, 0-5 (6 = no energy) \\
SSD/MCP PW & 9 & SSD (or MCP) pulse width \\
\hline
\end{tabular}

The JEDI hardware passes valid particle event data to the software for further analysis. The events pass through a First-In First-Out (FIFO). In order to understand the description of event processing that follows, it is important to understand the physical configurations of the timing circuits. On the event board (Fig. 13 center) there are 3 TOF ASIC devices, TOF1, TOF2, and TOF3, each of which measures time differences down to the sub-ns regime. TOF circuitry is used for two different purposes: (1) determining the entrance position of the incoming particle in conjunction with a time-delay anode that collects charge from the MCP (it also determines the position that the particle leaves the TOF sensor volume using a similar anode in the "stop" region of the MCP anode), and (2) determines the time-of-flight of the particles through the TOF sensor volume. In the description below, identifiers like Start0, Start5, Stop0 and Stop5 correspond to which end (0 or 5) of the time delay anode to which one side of the timing circuit is attached.

The valid event parameters pass through to the software with a First-In First-Out (FIFO) device. Each ion species event consists of several parameters, which are shown in Table 5. For energy events, only SSD data are valid. For events that trigger the Time-of-Flight system, TOF1, TOF2, and TOF3 are the raw values produced by the three TOF chips. TOF1 and TOF2 provide redundant measurements of the particle's time-of-flight. TOF1 measures the time between the Start0 and Stop0 pulses; TOF2 measures the time between the Start5 and Stop5 pulses. TOF3 measures the time between the Start0 and Start5 pulses; this provides the particle's position on the start anode. The "TOF" parameter in Table 5 provides the corrected TOF value, the average of TOF1 and TOF2. The start position is measured by TOF3. The stop position, i.e. the time between Stop0 and Stop5, is calculated in the FPGA as TOF2 + TOF3 - TOF1; the result is not reported in the event data. The start position and the stop position are used to calculate a start channel and a stop channel, respectively. The start and stop directional channel numbers are analogous to the six SSD energy directional channel numbers. The start, stop, and SSD directional channels are numbered in the same way, i.e., they should all have the same value for a given particle that is unscattered. The start and stop directional channel numbers are found from the start and stop positions via table lookup. The start and stop position threshold tables are uploadable parameters. Still considering Table 5, the MCP PH parameter is the pulse height of the analog sum of the Start0 and Start5 pulses. 
A baseline value is subtracted from the sum to form the pulse height; negative values are replaced with zero. The baseline is an uploadable parameter. The MCP PH Flag indicates whether a pulse was detected, i.e. it exceeded its commanded threshold. SSD Energy is the value of the selected energy channel. The energy channel selected is specified in the SSD Chan indicator. A baseline value is subtracted from the measured energy. There is a separate baseline for each channel; these are uploadable parameters. The SSD Flags indicate which SSD channels had pulses exceeding their commanded thresholds. SSD/MCP PW is the width of the SSD energy pulse for energy events or ion species events that have an SSD energy measurement. For ion species events with no SSD energy measurement (i.e. SSD Chan =6), SSD/MCP PW is the width of the MCP pulse.

The event board digitizes the TOF and energy and reads the events into a Field Programmable Gate Array (FPGA). The FPGA contains event processing logic and a processor. Some events are passed to software running on the processor for further analysis and science processing. The power supply board has housekeeping electronics and a Low Voltage Power Supply (LVPS). The support board contains a High Voltage (HV) power supply, non-volatile memory, and spacecraft interface electronics.

The processor, embedded within the FPGA, processes events that come to it through the FIFO. The processor uses lookup tables to channelize the data (Sect. 4.6) and captures a small fraction of the individual event parameters (derived from the information shown in Table 5) for monitoring the sensor performance and the fidelity of the channelization on the ground. Bench testing shows that the JEDI throughput of the JEDI processor (the number of events captured in the FIFO that the processor can process) is about 30,000 events per second against a design requirement of 10,000 events per second. When the input rate is higher than 30,000 events per second the processor does not process every event, but the architecture of the processing is carefully designed so that the processor accurately poles the incoming events. Because the electrons and ions are processed at different times, the electrons and ions do not compete with each other within the processor. Again, when the incoming event rate is greater than the rate at which the processor can process the events, the processor-channelized data must be renormalized using the numerous rate channels that are captured within the FPGA, and which can count at rates greater than $5 \times 10^{5}$. The rate channels are documented in Appendix A in Tables 12-15.

\subsubsection{Event Board Overview}

The Event board directly processes the sensor SSD and anode preamp output signals, and contains all the necessary analog and digital circuitry to process and store event information on an event-by-event basis. The energy signals from the six SSD preamplifiers and the MCP anode pulse height are processed in parallel peak-detect/discriminator circuit chains into a multiplexed analog to digital conversion (ADC) chain. The MCP anode signals are processed via constant-fraction discriminators (CFDs) and time-to-digital (TDC) circuitry; the measured time differences are converted into event look direction and particle velocity in the FPGA. FPGA-based event logic also determines which signals comprise valid ion and electron events and coordinates all event hardware processing timing. A soft-core processor clone (APL's Scalable Configuration Instrument Processor-SCIP; Hayes 2005) is also embedded in the FPGA to provide all command, control, telemetry, and data processing functions of the instrument. SRAM memory storage is provided on the board to support this processor. EEPROM and boot PROM support is provided on the Support Board. The Event board plugs into the Support and Power boards. 


\subsubsection{Support Board Overview}

The Support board provides a variety of support functions for the instrument. It also contains EEPROM and boot PROM accessible to the FPGA on the Event Board. The command and telemetry interface to the spacecraft is provided here. The board includes the high voltage power supply, which generates the necessary high voltage outputs for the sensor MCP and electron optics; maximum voltage is 3300 V. The Support board plugs into the Event and Power boards.

\subsubsection{Power Board Overview}

The Power board contains both the low and SSD bias voltage power supplies. The low voltage portion takes spacecraft primary power on a single 9 pin connector and generates $1.5 \mathrm{~V}$ (for FPGA core), $3.3 \mathrm{~V}$ (primarily for digital interface logic, memories, and TDCs), and $5 \mathrm{~V}$ (primarily for analog functions). A $15 \mathrm{~V}$ output powers the high voltage electronics on the support board. The board also switches power to the sensor cover actuator mechanism and generates and filters $100 \mathrm{~V}$ bias for the SSD detectors. The board plugs into both the Event and support boards.

\subsubsection{ASICS}

JEDI utilizes five different APL-developed rad-hard ASICs in its electronics (Paschalidis et al. 2002; Paschalidis 2006). It has three APL TOF-C ASICs to measure the "Start" (entrance) and "Stop" (exit) positions on the sensor timing anodes and the time-of-flight for ions traveling between the Start and Stop foils. The TOF ASICs, which also incorporate very fast constant fraction discriminator front ends, are configured to measure times between 0 and $32 \mathrm{~ns}$ with 50 ps resolution (anode positions) and between 0 and $160 \mathrm{~ns}$ with 200 ps resolution (time-of-flight). Each of JEDI's six look directions utilize a Quad Energy Chip (preamp/shaper) ASIC followed by a peak detector/discriminator ASIC to process the 4-pixel solid state detector (SSD) arrays. The capability of the Quad Energy Chip ASIC to handle shaping time constants $\geq 200 \mathrm{~ns}$ and energy dynamic range up to $25 \mathrm{MeV}$ was critical to meeting the wide count and energy dynamic range requirements. JEDI's control circuitry utilizes a 16-channel TRIO ASIC to multiplex and perform 10-bit analog to digital conversion of analog status information, and a number of Quad 8-bit DACs to set thresholds and control high voltage and SSD bias levels; the TOF ASICs communicate with the instrument FPGA via a parallel interface, while the Quad DAC and TRIO use serial I2C interfaces. The ASICs each require between 5 and $25 \mathrm{~mW}$, and all inherently meet performance requirements beyond $100 \mathrm{krad}$ radiation dose.

\subsubsection{Harnessing}

The sensor head is electrically connected to the electronics box via coaxial cables and twisted wire interfaces. These lines are fairly short in length (typically less than $10 \mathrm{~cm}$ ), and are covered by a thin EMI shield to extend the Faraday cage between the sensor and electronics housings. The instrument is electrically interfaced to the spacecraft via dedicated spacecraft-provided power and data cables. 


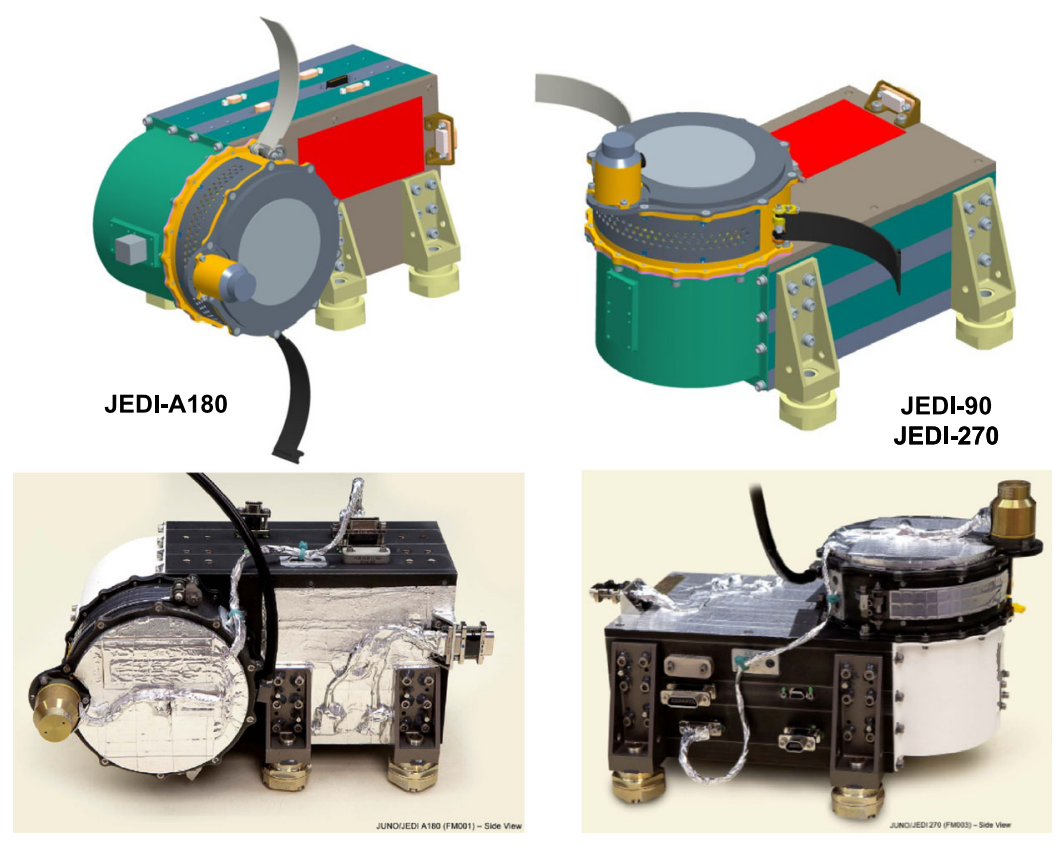

Fig. 14 Drawings and photographs of the external mechanical configurations of the JEDI instruments

\subsection{JEDI Mechanical Configuration}

\subsubsection{External Instrument and Mounting}

The external mechanical configurations of the two JEDI instrument configurations are shown in Fig. 14. The drawings show the instruments with their one-time deploy acoustic doors deployed, whereas the photographs show the doors closed. The mounting positions of the three JEDI instruments on the Juno spacecraft payload mounting plate are shown in Fig. 15. The numbers that designate the 3 JEDI instruments $(90,180,270)$ indicate which side of the spacecraft on which the sensors are mounted; they are the rough mounting positions in degrees angle within the $X-Y$ coordinate system of the spacecraft. The large "dish" in the center of the plate in Fig. 15 generally views towards Earth (and also roughly towards the sun). The solid blue panels are the solar panels that extend many meters away from the main body of the spacecraft.

Details of the mounting of JEDI-A180 are shown in Fig. 16. This is the sensor that views both towards the sun and away from the sun in a continuous plane. To minimize solar light contamination there is a 12 degree blockage in the 160 degree field of view, as indicated by Fig. 2.

Details of the mounting of JEDI-90 or JEDI-270 are shown in Fig. 17. The tilts of the instrument (10 degrees around one axis and 8 degrees around another) are needed to prevent the fields-of-view of the instruments from viewing glint off of the very long solar panels (a 20 degree keep-out-zone was established). It is this compromise that has degraded somewhat the optimum viewing with respect to the magnetic field that is shown in Fig. 4. Exact pointing information is provided in Appendix A. 


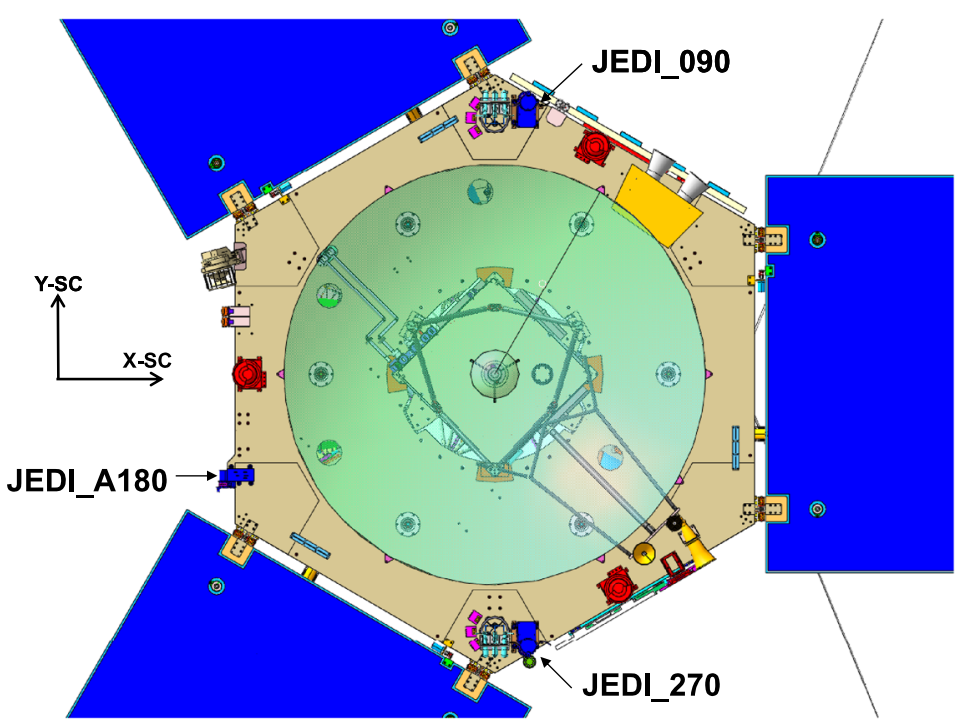

Fig. 15 Mounting positions of the 3 JEDI sensors on the Juno payload plate. The structure in the middle is the Juno high gain antenna that nominally points in the general direction of the sun and Earth during Jupiter orbital operations

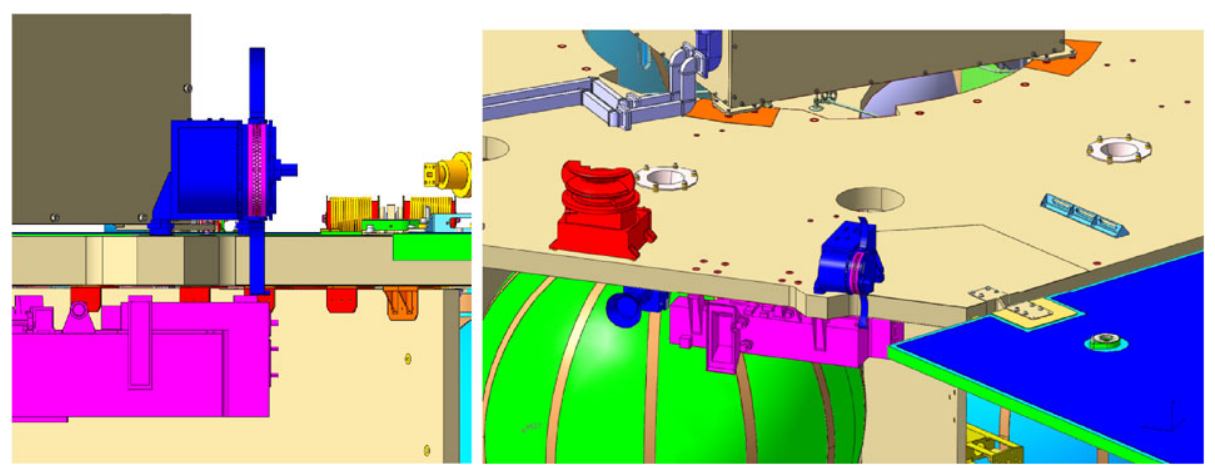

Fig. 16 Details of the mechanical mounting of JEDI-A180 on the Juno spacecraft

\subsubsection{Internal Structure}

The internal structure of each JEDI instrument is shown with the cutaway diagram in Fig. 18. There is TINI actuated pin puller that releases the spring-loaded doors. The figure shows some of the internal structure of the sensor, and the positioning of the three main electronics boards. Selected elements of the sensor are shown in Fig. 19. The upper left hand portion shows the anode board with the energy system mounted on to it. The metalized anode itself in the center shows 12 anode pads in the "start" portion (bottom) and 12 anode pads in the "stop" region. The anode pads are paired to generate 6 positions in the processing of the time delay along the string of anode pads. In the TOF assembly in the lower right, the electro static mirror diverts the secondary electrons from the start and stop foils down onto the MCP (see also Figs. 11 and 18). The TOF/MCP assembly in the lower right of Fig. 19 

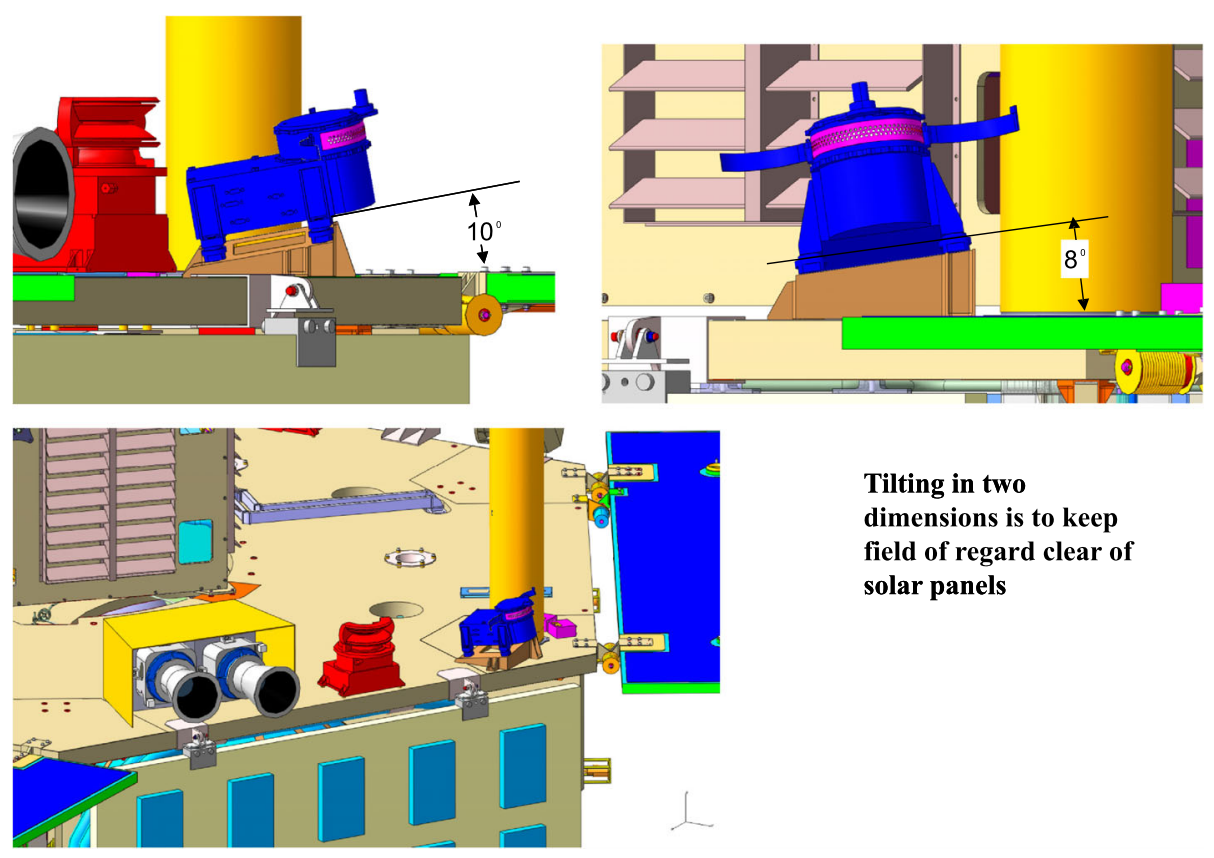

Tilting in two

dimensions is to keep

field of regard clear of

solar panels

Fig. 17 Details of the mechanical mounting of JEDI-90 on the Juno spacecraft. The mounting of JEDI-270 is very similar

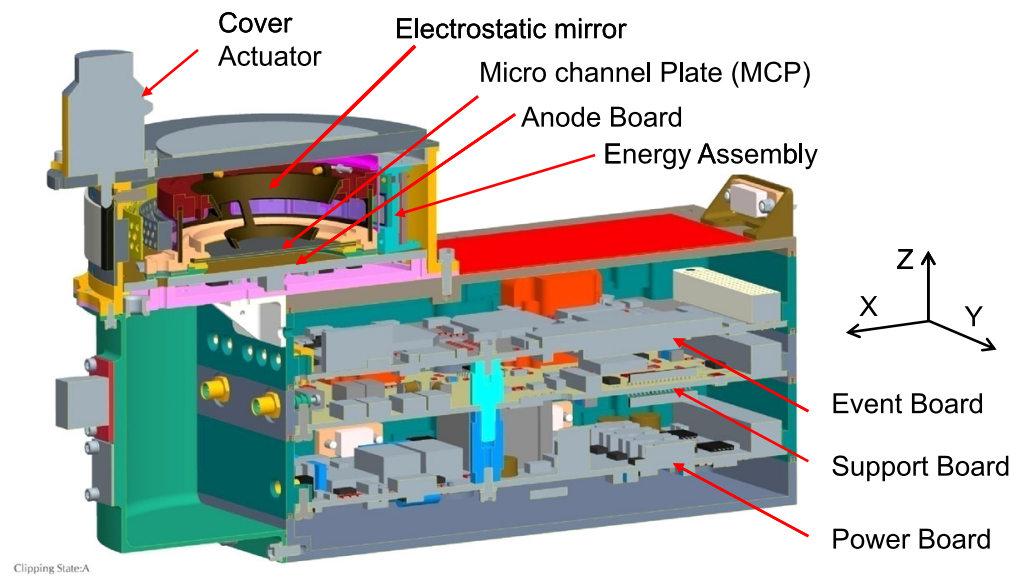

Fig. 18 The internal mechanical configuration of one of the JEDI instruments

has a top and a bottom piece that sandwich together to hold the start and the stop frames and foils, one of which can barely be seen through the gap in the bottom of the image. Figure 20 (top) shows the start and stop foil and holder on a storage mount. Technical specifications of the foils are given in Appendix A.

The collimator shown in Fig. 21 fits into the gap that is apparent in the bottom portion of the sensor assembly shown in the upper right of Fig. 19. The collimator consists of 5 blades 

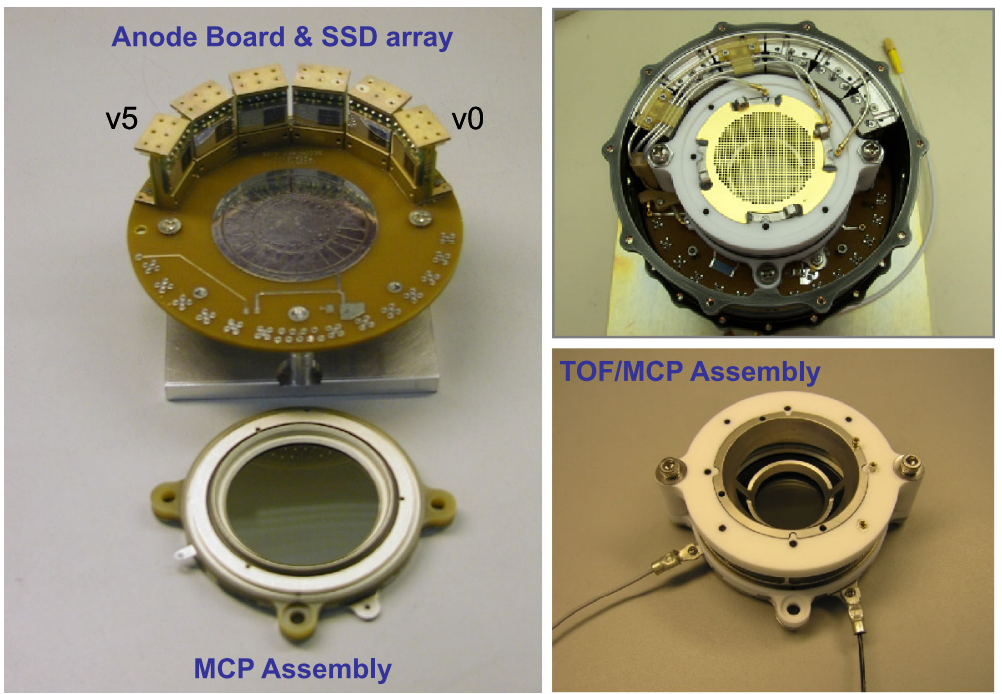

Fig. 19 Photographs of various internal components of one of the JEDI sensors

Fig. 20 (Top) start and stop foils mounted on $\mathrm{Ni}$ grids that are, in turn, mounted onto the foil mounting frames. (Bottom) Collimator foil mounted on a stainless steel grid that is, in turn, mounted on the removable blade from the collimator

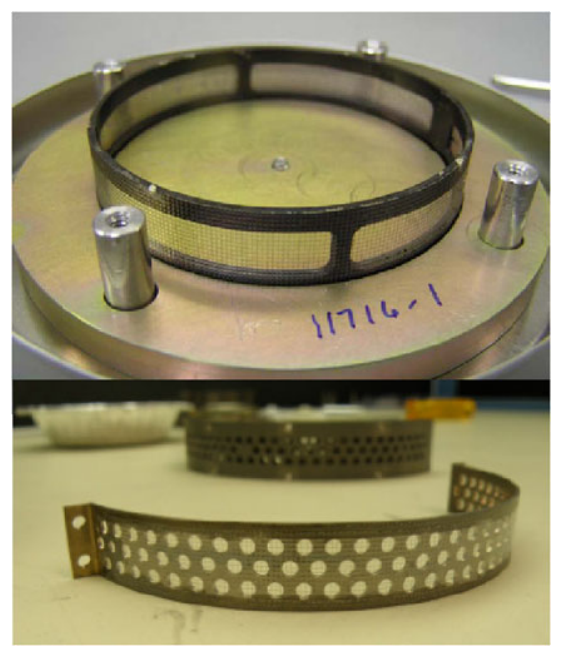

of Tungsten-Copper mixture, each with a hexagonal array of three rows of aligned holes for a total of about 90 holes (Fig. 20, bottom). The middle blade holds the collimator foil, an image of which is shown in Fig. 20 (bottom). The sizes of the holes on each blade are graded according to distance of the blade to the center of the symmetry axis of the cylindrical sensor volume. Further technical specifications are given in Appendix A.

In designing this collimator, there is a balance to be struck between having a large geometric factor and eliminating all side lobes (making sure that the acceptance pattern of collimator is single valued. The choices that we made for JEDI leave residual side lobes; the worst case is shown in Fig. 22, corresponding to a side response of about $1 \%$ of the main directional response. In this figure, massive and randomly positioned and randomly oriented rays of light have their origins on the surface of just one of the solid state detectors. 
Fig. 21 The JEDI multiple-hole collimator with 5 blades

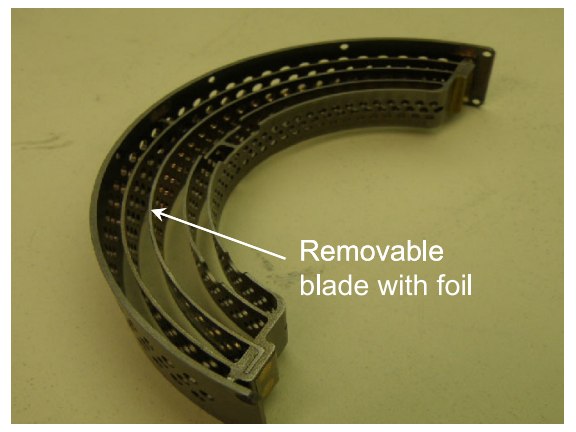

Fig. 22 The viewing response of the JEDI collimator showing the worst case result for the occurrence of side lobes. The central peak is the main response, and the sprinkle of points to the left represents the side lobes. The integrated side lobe response represents about $1 \%$ of the main response

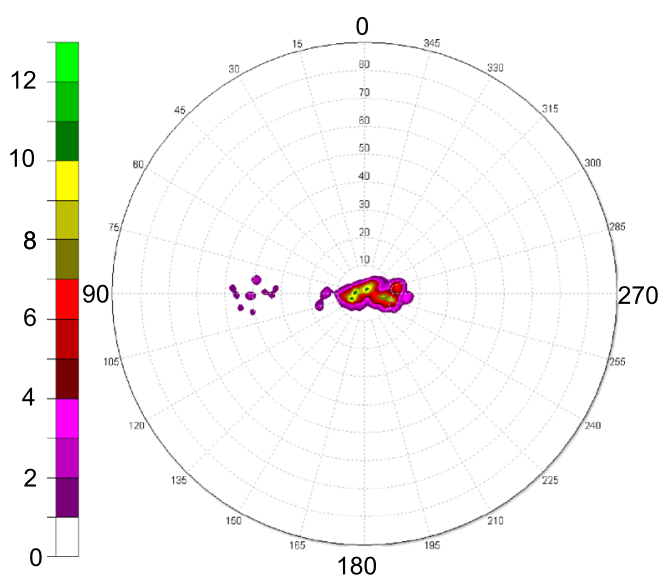

The figure shows the density of rays that are able to get out of the instrument. The central blob is the primary response of the collimator, and the points to the left comprise the side lobe.

\subsection{JEDI Detectors}

\subsubsection{Solid State Detectors (SSD's)}

One of 6 of the SSD holders per JEDI instrument is shown in Fig. 23. The side of the holder that is shown holds a single SSD, manufactured by Canbarra, with 4 pixels, 2 electron pixels and 2 ion pixels. Each large pixel is about $0.40 \mathrm{~cm}^{2}$ and each small pixel is about $0.02 \mathrm{~cm}^{2}$, yielding sensitivity ratio of about 20. The electron pixels are covered with an aluminum flashing 2 microns thick. The GEANT4 simulation in Fig. 24 shows that, with $20 \mathrm{keV}$ discrimination on the SSD output, electrons with energy starting at about $25 \mathrm{keV}$ and above can be measured, whereas protons with energy of $250 \mathrm{keV}$ and above can to be detected. The solid state detector is 500 microns thick with a dead layer, relevant for the ion side, of about $500 \AA$. The hanger itself is made of Tungsten-Carbon and is $0.25 \mathrm{~cm}$ thick. It represents one part of the effort to shield the SSD's from most directions with $0.5 \mathrm{~cm}$ Tungsten-Carbon for background control. On the back side of the hanger is a small board that contains the Energy ASICS described in Sect. 4.3. How the SSD hangers are mounted into the array that is needed for the JEDI sensor is shown in Fig. 19 (upper left). 
Fig. 23 One of 6 Solid State Detector (SSD) hangers in each JEDI instrument showing the mounted SSD with 4 pixels. Figure 19 shows how these hangers are mounted within the instrument
Fig. 24 GEANT4 study of the effectiveness of the 2 micron aluminum flashing on the electron SSD pixels in minimizing the detection of energetic protons while still allowing the sensor to measure electrons down to about $25 \mathrm{keV}$ (for a $20 \mathrm{keV}$ SSD discrimination level)
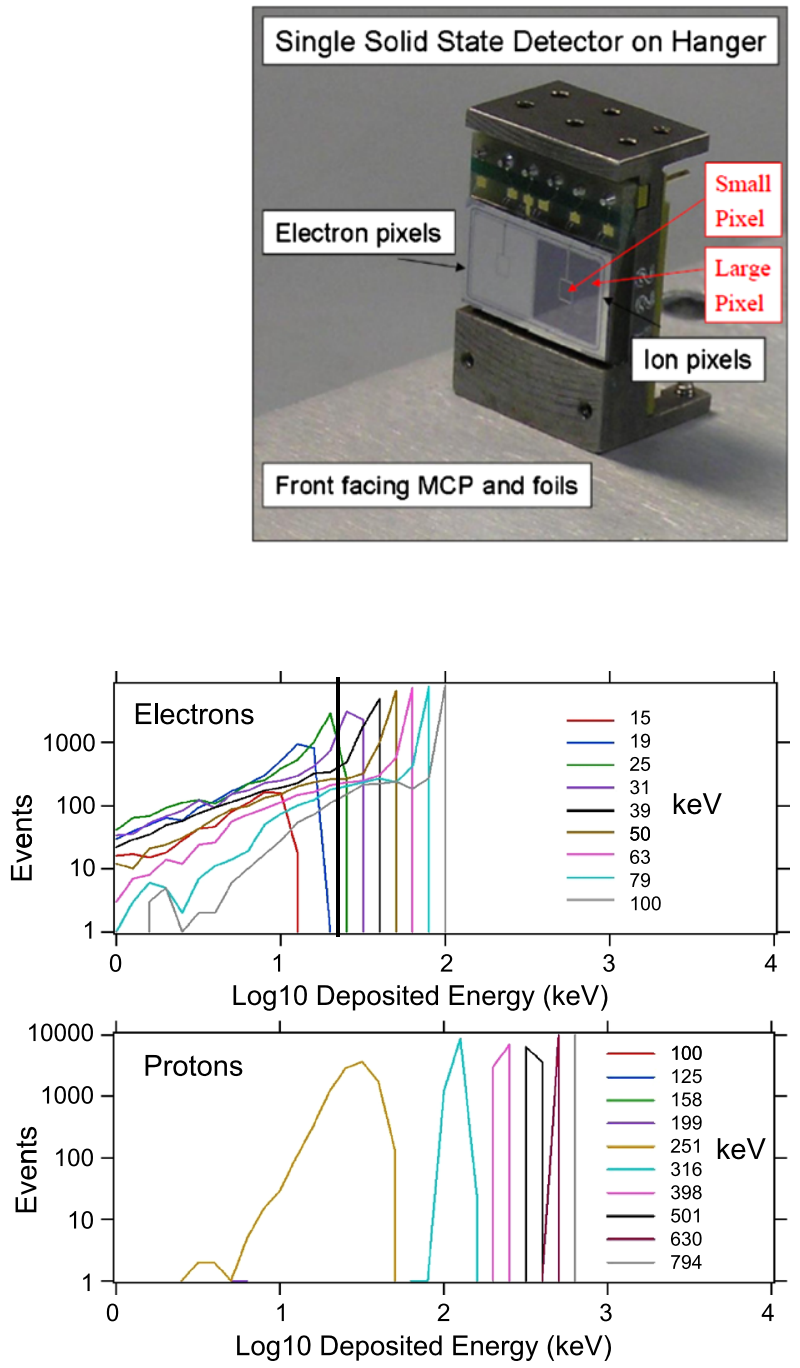

Microchannel Plates (MCP) The single microchannel plate stack (MCP) within each JEDI (Fig. 19, lower left) comprises two $5 \mathrm{~cm}$ diameter circular plates mounted, with a small gap between them, in the chevron configuration. The stack is operated with a total potential drop of between 1800 and $2300 \mathrm{~V}$, and is used with a gain of several $\times 10^{6}$. The cloud of electrons coming out of the stack is collected by a segmented anode (Fig. 19 upper left), with 12 segments in the "start" region, and 12 segments in the "stop" region. Discrete inductors between the segments cause a time delay for the charge to be collected on both ends of the 12 -segment array that is proportional to the position along the array where the electrons are collected. The FPGA forms 6 viewing sectors from the information received from that timing information. The gap between the bottom of the MCP stack and the anode is $0.25 \mathrm{~cm}$, and the potential difference that collects the electron cloud is $\sim 100 \mathrm{~V}$. 
Table 6 Onboard Data Products per hardware mode

\begin{tabular}{lll}
\hline Electron energy & Ion energy & Ion species \\
\hline $\begin{array}{lll}\text { Electron Energy Spectra } \\
\text { Basic Rates (Electron Energy) }\end{array}$ & $\begin{array}{l}\text { Ion Energy Spectra } \\
\text { Basic and Diagnostic Rates } \\
\text { (Ion Energy) }\end{array}$ & $\begin{array}{l}\text { Proton (and Non-Proton) Rates } \\
\text { Basic and Diagnostic Rates (Ion } \\
\text { Species) }\end{array}$ \\
Raw Event Data (Electron Energy) & Raw Event Data (Ion Energy) & $\begin{array}{l}\text { Raw Event Data (Ion Species) } \\
\text { Priority Event Data (Ion Species) }\end{array}$ \\
\hline
\end{tabular}

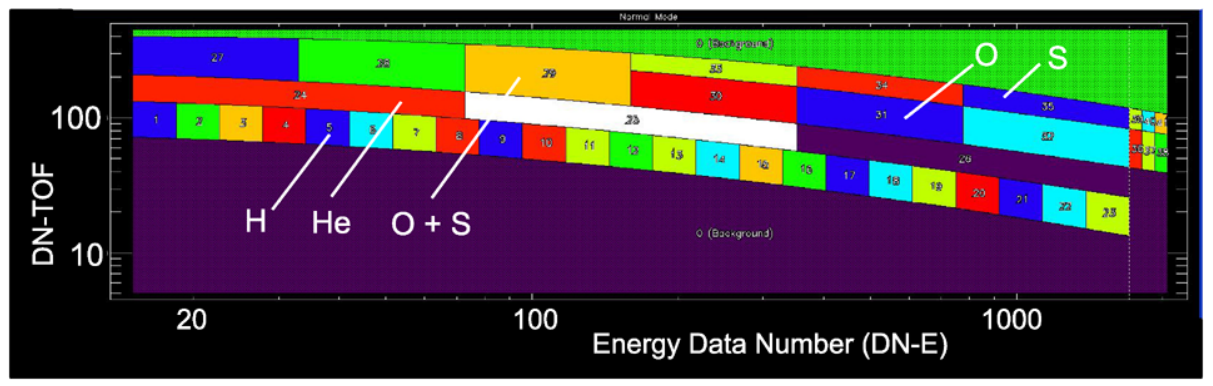

Fig. 25 The JEDI Time-of-Flight $\times$ energy $($ TOF $\times$ E) channels as they are configured in the JEDI-internal $\mathrm{TOF} \times \mathrm{E}$ data number matrix

\subsection{JEDI Internal Operations, Operational Modes, and Data Products}

Each SSD has electron and ion pixels. There is only one analog electronics processing chain per SSD. Consequently, to collect both electrons and ions, the hardware must be timemultiplexed between the electron and ion detectors. Similarly, the event processing logic is switched between modes that measure ion energy vs. ion species. The hardware is timemultiplexed between three possible modes: electron energy, ion energy, and ion species. These modes are defined in Table 6. For the electron energy mode and the ion energy mode, the JEDI software sorts the SSD energy parameter into a one-dimensional array of numbers that represent the electron or ion energy spectra, with a large number of spectral bins for "high resolution" spectra" and a smaller number of spectral bins for "low resolution" spectra. For the ion species mode, one 2-dimensional TOF $\times \mathrm{E}$ array is used for events that have both a TOF and an energy to sort the events according to mass and energy (see Fig. 25) and another 2-dimensional array is used for event that have only TOF information (TOF and Pulse Height) to similarly sort the events according to mass and TOF (or equivalently Energy/nucleon; See Fig. 26).

The JEDI software divides each spacecraft spin into 60 evenly spaced spin sectors. As the spin rate varies, the duration of a sector varies accordingly. The spin starts, i.e. sector 0 starts, when the spacecraft's inertial spin phase is zero. Inertial spin phase is defined as the angle between (i) the projection of ecliptic north in spacecraft $x y$ plane and (ii) the $x$-axis in the direction of spacecraft spin irrespective of the mounting configurations of the 3 JEDI instruments. The spacecraft provides spin rate and phase data to JEDI. JEDI maintains an internal spin model. At the start of each internal spin, the JEDI software calculates the current phase from the most recently received spacecraft phase data; any difference from zero constitutes a phase error. Based on the phase error and current spacecraft spin rate, 
Fig. 26 The JEDI

Time-of-Flight $\times$ Pulse-Height $(\mathrm{TOF} \times \mathrm{PH})$ channels as they are configured in the JEDI-internal $\mathrm{TOF} \times \mathrm{PH}$ data number matrix. The gap between the protons and the heavy ions is intended to minimize the mixing of species, and can be adjusted by uploading new tables

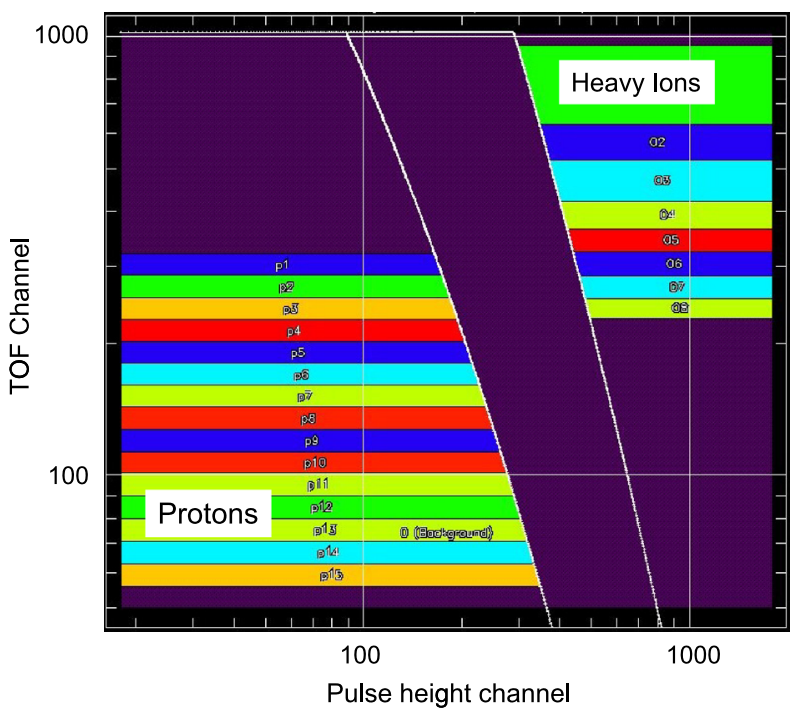

Subsector 1

Subsector 2

Subsector 3

Fig. 27 Structure of the rotational (and timing) subsectors in the JEDI data accumulation scheme that allows us to sub-commutate in a flexible manner between the 3 different species collection modes: electron spectra, ion spectra, and ion species. One can choose to use a cyclic of combination of modes within these subsectors. There are 60 sectors per spin, and so this pattern of subsectors occurs 60 times per spin

the JEDI software calculates a sector duration that reduces the phase error. (Note: if no spacecraft data has been received lately or it is invalid, a nominal 30 second spin period is used.) On startup, it will take several spins for the internal spin model to eliminate its phase error with the actual spacecraft spin. Once the spin model and the actual spin are in phase, they will stay in phase as the spacecraft spin rate varies. Each sector is further divided into three subsectors. The first subsector is long, 1/2 of a sector. The last two subsectors are short, 1/4 of a sector each (See Fig. 27). As with sectors, subsector timing varies with the spin rate. The sensor hardware can be placed in a different mode during each subsector. The dark bars in the figure represent a fixed dead-time for switching between hardware modes. The pattern of modes in each subsector is commandable. Any subsector may collect data in any mode. Each pattern collects different data in different proportions. For example, setting subsector 1 to electron energy and subsectors 2 and 3 to ion species collects electron energy $1 / 2$ of the time and ion species the rest of the time; ion energy is not collected at all. Note: if two adjacent subsectors have the same mode, there will still be a dead-time between the subsectors.

Table 7 documents the numerous JEDI hardware modes and parameter settings that can be set by command. Several "standard" settings (setting all of the various parameters shown in the table) are generated by running one of several internal instrument "macros" during the JEDI turn-on sequence or by external command at other times. As an example, optimum threshold settings have some sensitivity to temperature, and onboard macros are embedded within the instrument for several temperatures over the operational range. 
Table 7 JEDI Hardware modes

\begin{tabular}{|c|c|c|c|}
\hline Resource & Electron energy & Ion energy & Ion species \\
\hline Event Trigger & \multicolumn{2}{|l|}{ Set energy trigger } & Set TOF trigger \\
\hline $\begin{array}{l}\text { TOF CFD } \\
\text { Thresholds }\end{array}$ & \multicolumn{3}{|l|}{ Set TOF CFD thresholds } \\
\hline $\begin{array}{l}\text { TOF Pulse } \\
\text { Height Threshold }\end{array}$ & \multicolumn{2}{|c|}{ N/A: set TOF pulse height threshold to $\max$} & $\begin{array}{l}\text { Set TOF pulse height } \\
\text { threshold }\end{array}$ \\
\hline $\begin{array}{l}\text { Electron vs. Ion } \\
\text { Detector }\end{array}$ & Set electron source & Set ion source & $\begin{array}{l}\text { Set ion (or electron) } \\
\text { source }\end{array}$ \\
\hline Pixel Size & $\begin{array}{l}\text { Set pixel size selected for } \\
\text { electron }\end{array}$ & $\begin{array}{l}\text { Set pixel size selected for } \\
\text { ion energy }\end{array}$ & $\begin{array}{l}\text { Set pixel size selected for } \\
\text { ion species }\end{array}$ \\
\hline $\begin{array}{l}\text { Energy Channel } \\
\text { Enable/Disable }\end{array}$ & $\begin{array}{l}\text { Set energy mask for } \\
\text { electron and selected pixel } \\
\text { size }\end{array}$ & $\begin{array}{l}\text { Set energy mask for ion } \\
\text { and selected pixel size }\end{array}$ & $\begin{array}{l}\text { Set energy mask for ion } \\
\text { and selected pixel size }\end{array}$ \\
\hline $\begin{array}{l}\text { Energy } \\
\text { Thresholds }\end{array}$ & $\begin{array}{l}\text { Set energy discriminator } \\
\text { thresholds for electron and } \\
\text { selected pixel size }\end{array}$ & $\begin{array}{l}\text { Set energy discriminator } \\
\text { thresholds for ion and } \\
\text { selected pixel size }\end{array}$ & $\begin{array}{l}\text { Set energy discriminator } \\
\text { thresholds for ion and } \\
\text { selected pixel size }\end{array}$ \\
\hline Energy Baselines & $\begin{array}{l}\text { Set energy baselines for } \\
\text { election and selected pixel } \\
\text { size }\end{array}$ & $\begin{array}{l}\text { Set energy baselines for } \\
\text { ion and selected pixel size }\end{array}$ & $\begin{array}{l}\text { Set energy baselines for } \\
\text { ion and selected pixel size }\end{array}$ \\
\hline Coincidence & \multicolumn{3}{|l|}{ Set event coincidence window } \\
\hline $\begin{array}{l}\text { Multiple Hit } \\
\text { Reject }\end{array}$ & $\begin{array}{l}\text { Enable/disable multiple } \\
\text { hit reject for electron }\end{array}$ & \multicolumn{2}{|c|}{ Enable/disable multiple hit reject for ion } \\
\hline Valid Event & N/A & N/A & $\begin{array}{l}\text { Select valid TOF chips for } \\
\text { ion species }\end{array}$ \\
\hline
\end{tabular}

\subsubsection{Diagnostic and Test Support}

JEDI can inject pulses into the preamps of the TOF start, TOF stop, and SSDs for test purposes. The rate of the pulses is either controlled on-board or with an external pulse generator; the latter is only available during ground testing. Both a start and a stop pulse are generated. The rate and the start to stop delay are set by command. The start pulse can be sent to TOF start; the stop pulse can be sent to TOF stop and the SSDs. The TOF start, TOF stop, and SSD pulses can be enabled or disabled individually by command. The JEDI hardware can be commanded to measure SSD energy channel or MCP pulse height baseline values instead of doing its normal event processing. The results appear in the event FIFO with the relevant information shown in Table 5. SSD Energy contains the baseline value direct from the electronics, i.e., without the current baseline removed. SSD Chan indicates the directional channel being measured. Note that when measuring the MCP pulse height baseline, the values will appear in SSD Energy and the SSD Chan will indicate 6 (a default; not a real direction).

\subsubsection{Onboard Data Structures and Products}

The data products generated by the JEDI software (Table 6) are each organized into three types depending on their integration time, fast, medium, or slow, as documented in Table 8. The duration of fast, medium, and slow integrations is set by command. The command has three arguments, S, N1, and N2. S specifies the number of sectors to integrate fast data 
Table 8 JEDI Onboard data products and their integration times

\begin{tabular}{|c|c|c|c|}
\hline Type & Data product & Integration time (Sectors) & Multi-spin \\
\hline \multirow[t]{9}{*}{ Fast } & Electron Energy Basic Rates & $\mathrm{S}$ & Integrate \\
\hline & Ion Energy Basic Rates & & Integrate \\
\hline & Ion Energy Diagnostic Rates & & Integrate \\
\hline & Ion Species Basic Rates & & Integrate \\
\hline & Ion Species Diagnostic Rates & & Integrate \\
\hline & Low Energy-Res./High Time-Res. Ion Spectra & & Integrate \\
\hline & Low Energy-Res./High Time-Res. Electron Spectra & & Integrate \\
\hline & $\begin{array}{l}\text { Low-Res./High Time-Res. TOF } \times \text { Energy Proton } \\
\text { Rates }\end{array}$ & & Integrate \\
\hline & $\begin{array}{l}\text { Low-Res./High Time-Res. TOF } \times \text { Pulse Height } \\
\text { Proton Rates }\end{array}$ & & Integrate \\
\hline \multirow[t]{8}{*}{ Medium } & High Energy-Res./Low Time-Res. Ion Spectra & $\mathrm{S} \times \mathrm{N} 1$ & Integrate \\
\hline & High Energy-Res./Low Time-Res. Electron Spectra & & Integrate \\
\hline & $\begin{array}{l}\text { High-Res./Low Time-Res. TOF x Energy Proton } \\
\text { Rates }\end{array}$ & & Integrate \\
\hline & $\begin{array}{l}\text { High-Res./Low Time-Res. TOF x Pulse Height } \\
\text { Proton Rates }\end{array}$ & & Integrate \\
\hline & Priority Events & & Decimate \\
\hline & Raw Electron Energy Event Data & & Decimate \\
\hline & Raw Ion Energy Event Data & & Decimate \\
\hline & Raw Ion Species Event Data & & Decimate \\
\hline \multirow[t]{2}{*}{ Slow } & TOF $\times$ Energy Non-Proton Rates & $\mathrm{S} \times \mathrm{N} 1 \times \mathrm{N} 2$ & Integrate \\
\hline & TOF $\times$ Pulse Height Non-Proton Rates & & Integrate \\
\hline \multirow[t]{2}{*}{ Spin } & Auxiliary Data & 60 (i.e. 1 spin) & \\
\hline & Magnetometer Data & & Decimate \\
\hline
\end{tabular}

products. N1 specifies the number of fast integrations that make up a medium integration; in other words, medium data products are integrated for $\mathrm{S} * \mathrm{~N} 1$ sectors. Similarly, N2 specifies the number of medium integrations that make up a slow integration, i.e. slow data products are integrated for $\mathrm{S} \times \mathrm{N} 1 \times \mathrm{N} 2$ sectors. The basic and diagnostic rates identified in Tables 6 and 8 are shown in Appendix A in Tables 12-15. The ion and electron "spectra" are energychannelized rates with the highest energy resolution channels documented in Appendix B, in Tables 16, 17. Some data products documented in Table 8 have reduced spectra where subsets of channels for the highest resolution spectra are summed. The "priority event" data represents events that are channelized with the tables shown in Figs. 25 or 26, and the resulting channels for the highest resolution data is shown in Appendix B in Tables 18, 19, 20. The "raw event data" are subsets of the information shown in Table 5 for a small fraction of the individual events that are processed in the processor.

The raw event data allows us to build (over several hours, given limitations in telemetry) displays on the ground like the two bottom panels of Fig. 12 (JEDI examples are shown in Sect. 5 (Figs. 30 and 33). In order to diagnose all regions of the TOF $\times \mathrm{E}$ and $\mathrm{TOF} \times \mathrm{PH}$ arrays, the events that are telemetered to the ground can be selected (by setting a command parameter) according to a rotating priority scheme that cycles through (with highest priority) the different regions of TOF $\times \mathrm{E}$ and $\mathrm{TOF} \times \mathrm{PH}$ arrays. 
Fig. 28 The efficiency of detection of various particle species as a function of incoming total energy

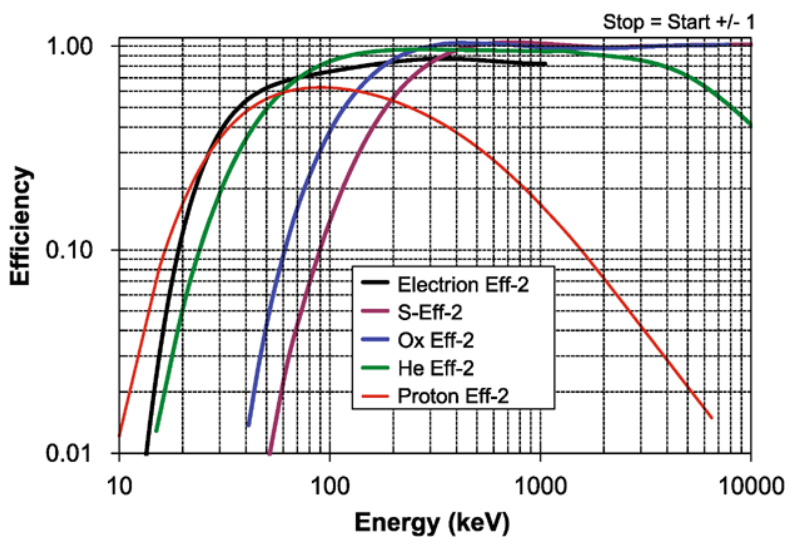

\section{JEDI Calibration and Performance}

\subsection{Efficiencies, Channel Characteristics and the Factors that Affect Them}

The efficiency by which the typical JEDI instruments measure electrons, protons, helium ions, oxygen ions and sulfur ions as a function of incoming energy (keV) is shown in Fig. 28. The roll-off in ion efficiency as one goes from intermediate to lower energies occurs primarily as a result of scattering; particles come into the collimator on valid trajectories but scatter in the collimator foil or the active start foil, change their directions of flight, and subsequently strike a non-sensing part of internal sensor volume. The roll-off in electron efficiency as one goes from intermediate to lower energies also has a scattering component to it, but is dominated by the electron interactions with the $2 \mathrm{~mm}$ aluminum flashing on the electron sensors. The ion roll-off in efficiency as one goes from intermediate energies to very high energies for protons and helium ions occurs as a result of the reduction in the efficiency for the generation of secondary electrons within the start foil and the stop foil. The efficiency of secondary electron generation for ions (the foils are not used to detect the electrons) scales roughly as the stopping power $(d E / d x$; often characterized with the units $\mathrm{keV} / \mathrm{micron}$ ), and the stopping power of protons and helium ions fall substantially for higher energies. The total sensitivity for measuring a specific species at a specific energy is determined by the efficiency $(\varepsilon)$ times the geometric factor $(\varepsilon \cdot G$; where $G$ has the units $\left.\mathrm{cm}^{-2} \mathrm{sr}^{-1}\right)$. Specifically, Intensity $\left(I\right.$ : particles $\left.\mathrm{cm}^{-2} \mathrm{sr}^{-1} \mathrm{keV}^{-1}\right)=R /\left[\varepsilon \cdot G \cdot\left(E_{2}-E_{1}\right)\right]$, where $R$ is rate (counts/s) for a specific channel and $E_{2}$ and $E_{1}$ are the energy boundaries of specific energy channel. One of the trade-offs that may be made with the setting of an on board parameter is the degree to which the start sector matches the stop sector (the start and stop sectors may not match due to scattering). One may require that "stop $=\operatorname{start} \pm n$ ", where " $n$ " may be 0,1 or 2 . For $n=0$ the measurements are the cleanest and with the lowest time dispersion. For high values of $\mathrm{n}$ the efficiency is greater. Figure 28 was made under the assumption that $n=1$.

The typical characteristics of the JEDI energy-species channels (the high energyresolution channels) is shown in Appendix B in Tables 16-20, which provide the energy window for each energy channel, the geometric factor $(G$, for large pixels only for Tables 16-19, where the SSD's are involved), and the efficiency (the same efficiency that is shown in Fig. 28). We emphasize that Tables 16-20 are "typical" characteristics. The specific channel characteristics (energy windows, efficiencies) will vary somewhat from JEDI unit to JEDI unit and from channel to channel. 
Fig. 29 The response of the JEDI SSD's to the JEDI-procured Ba133 radiation source. Such spectra are used to calibrate the internal energy data number (DN) into energy in $\mathrm{keV}$. The peaks are labeled with the data numbers for the particular SSD and processing chain that is being tested. Those peaks are to be compared with the source energies in Table 9

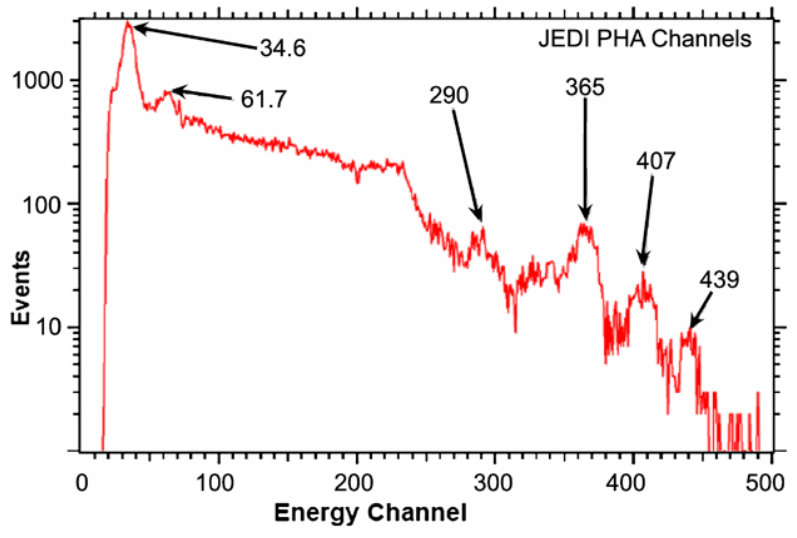

\subsection{Calibration Procedures and Facilities}

The response of the JEDI instruments is complicated, and our understanding is based on a coordinated array of approaches, specifically: (i) bench testing of channel gains and other characteristics based on calibrated pulse inputs, (ii) calibrations using particle accelerator beams, (iii) calibrations using radiation sources, (iv) simulations of particle interactions with matter using such tools as GEANT4, and (v) geometric calculations. Two particle accelerators were used for calibrating JEDI: (1) the JHU/APL ion particle accelerator that generates narrow ion beams of $\mathrm{H}, \mathrm{He}, \mathrm{O}$ ( $\mathrm{N}$ often used as proxy), $\mathrm{Ar}$, and other ions species from energies as low as about $12 \mathrm{keV}$ up to $170 \mathrm{keV}$; and (2) the GSFC Van de Graff, accelerator that generates electron and ion species beams from $\sim 100 \mathrm{keV}$ to $>1 \mathrm{MeV}$. Two different radiation sources were used. These sources are a Barium Ba133 source and a degraded Americium Am241 radiation source (the source is degraded by placing a thin mylar foil between the source and the sensor, which yields a very broad spectrum of alpha particle energies). To perform the calibrations we have procured sources that are configured so as to completely fill the fields-of-view of the JEDI sensor (we call these sources the "Geordi" sources given that they look much like the artificial eyes worn by Geordi La Forge in the television show: Star Trek, The Next Generation). Because the sources fill the JEDI field of view, all 6 look directions are calibrated simultaneously.

The Ba133 source provides the information needed to convert internal SSD energy data numbers (dn-E) into energy (keV) for all 24 SSD pixels (6 large ion pixels, 6 small ion pixels, 6 large electron pixels, and 6 small electron pixels. A typical Ba133 spectrum from the JEDI instrument is shown in Fig. 29. The local maxima correspond to specific X-ray or electron emission lines coming from the Ba source. The specific lines that are used to calibrate JEDI are provided in Table 9. Note that the electron lines are specific to the procured JEDI sources because account must be taken of the losses within the binding agent used to manufacture the sources.

The degraded AM241 provides a broad energy-distribution alpha source that tests the Time-of-Flight system and energy system simultaneously for all 6 TOF $\times$ PH look directions, all 6 of the TOF $\times$ E large-pixel look directions, and all 6 of the TOF $\times E$ small pixel look directions. A typical degraded Am241 TOF $\times \mathrm{E}$ spectrum is shown in Fig. 30, with a large pixel to the left and a small pixel to the right. Here we see that essentially the entire energy range is tested and the shortest, most challenging portion ( $\sim 5$ to $\sim 50 \mathrm{~ns})$ of the full JEDI TOF range $(\sim 5$ to $\sim 160 \mathrm{~ns})$ is also tested. These 
Table 9 Ba133 Selected emission lines

\begin{tabular}{ll}
\hline Species & Peak energy (keV) \\
\hline & Ba133 (JEDI-source) \\
X-Rays & 31 \\
X-Rays & 81 \\
Electrons & $267(247)$ \\
Electrons & $320(301)$ \\
Electrons & $349(331)$ \\
X-Rays & 384 \\
\hline
\end{tabular}

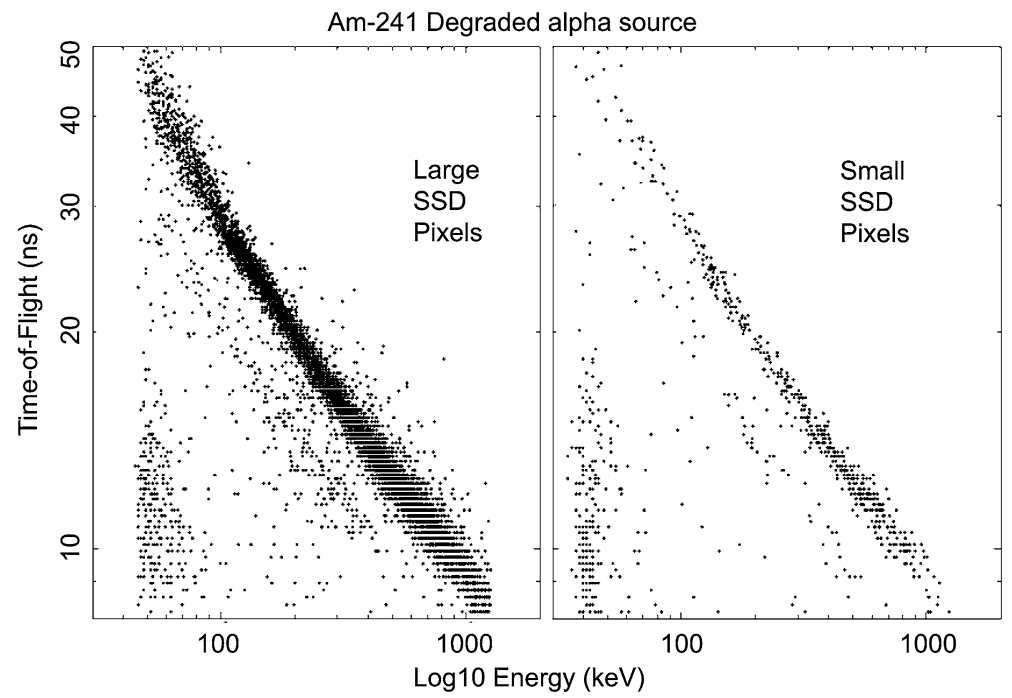

Fig. 30 The response of the JEDI TOF $\times$ E system to the JEDI-procured and degraded AM241 alpha radiations source. The $\sim 5 \mathrm{MeV}$ emitted alpha particles is degraded (and spread over a broad range of energies) with a mylar foil. This plot tests the response of both the energy and the TOF system and, by bootstrapping the Ba133 calibrations, allows one to determine the conversion from TOF data number to TOF in ns for the faster times of flight. The left panel is for a large SSD pixel, and the right panel is for a small SSD pixel, showing that the small pixels provide equivalent information at reduced sensitivity

spectra test uniformity across all look directions and all units, and provide a back up method of determining how to convert internal TOF data numbers (dn-TOF) into true TOF (ns) by bootstrapping off of the Ba133 determination of the true energies. The reason that accuracy in the determination of the TOF in ns can be established with this method is that, because $E=m V^{2} / 2$, the relative errors in the determination of the TOF $(\triangle \mathrm{TOF} / \mathrm{TOF})$ is the square root of the relative error in the determination of energy; that is it scales as $(\Delta E / E)^{0.5}$. The ghost signatures in the TOF $\times E$ displays is due to a combination of scattering of ions within the sensor (particularly off of the conelike electrostatic mirror structure), SSD edge effects, and high energy penetrations of grids.

In the next section, we use mostly exhibits from our accelerator beam calibrations to demonstrate that the JEDI design achieves its required performances. 


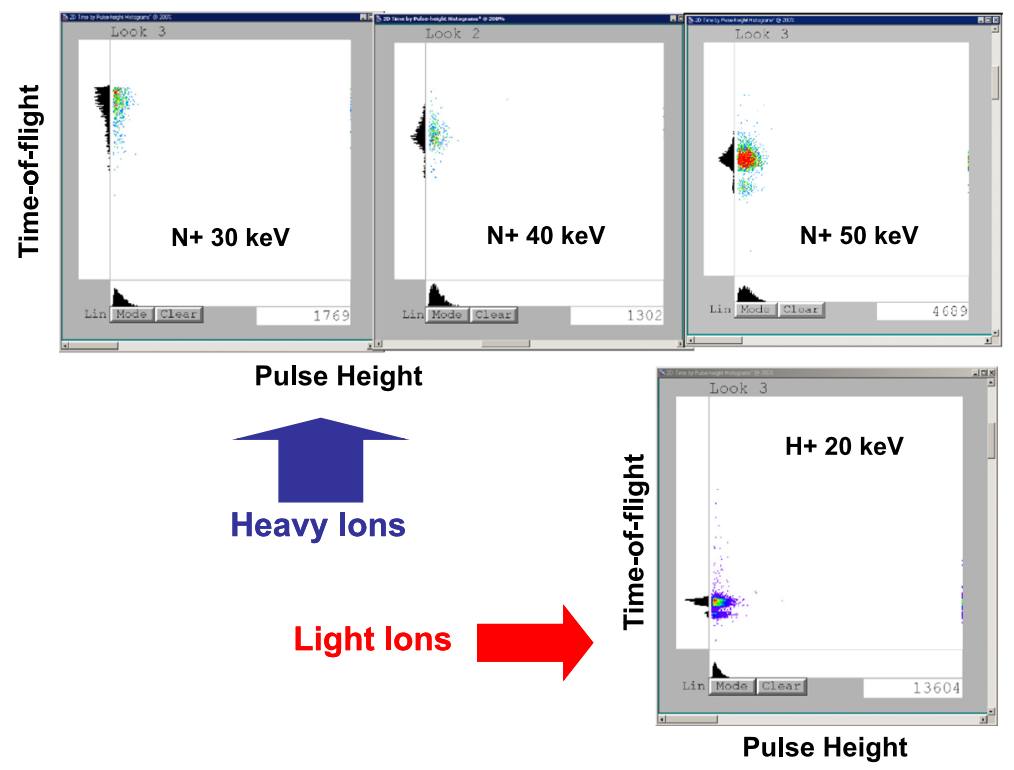

Fig. 31 TOF $\times$ Pulse-Height screen captures obtained during accelerator calibration runs showing that JEDI meets minimum energy requirements for measuring protons and oxygen (nitrogen is a proxy)

\subsection{JEDI Performance Verification}

In this section we show various selected exhibits from our JEDI calibration runs that we used to verify the performance of the JEDI instruments.

Figure 31 shows 4 different Time-of-Flight $\times$ Pulse Height $(\mathrm{TOF} \times \mathrm{PH})$ screen captures obtained during beam calibration at the JHU/APL ion accelerator that demonstrates that the JEDI instruments measure oxygen ions (nitrogen is used as a proxy) below the required $50 \mathrm{keV}$ and protons down to the required $20 \mathrm{keV}$.

Figure 32 shows 2 different Time-of-Flight $\times$ Energy $(\mathrm{TOF} \times \mathrm{E})$ displays, one from the GSFC ion accelerator facility (left) and one from the JHU/APL ion accelerator facility, that demonstrate (together with Fig. 31) that the required ion energy ranges are achieved and that the TOF $\times \mathrm{E}$ function achieves the mass discrimination capabilities that are required. For the display on the right, a degraded Am241 spectrum (alpha particles) is shown in addition to the accelerator beam results. The slight shift between the Am241 and beam responses is due to variations in the data-number to energy conversions for different solid state detector chains.

Figure 33 shows that the JEDI Time-of-Flight $\times$ Pulse Height $($ TOF $\times$ PH) function separates light ions (protons) from heavy ions (nitrogen is used as a proxy on the right). This function is used for the lowest energy ion measurements. The separation is not (and is not expected to be) nearly as clean as the separation that is achieved with the TOF $\times \mathrm{E}$ function. The approach that is taken for in flight measurements is to sample the heavy ions only at the highest pulse height region of the distribution in order to get a good sampling of the heavy ions uncontaminated with protons. Figure 26 shows how that sampling is achieved with the onboard channel tables (the regions just between the proton and heavy ion channels are not sampled), and the gap size can be modified (with new tables) as we gain experience with each unit. 

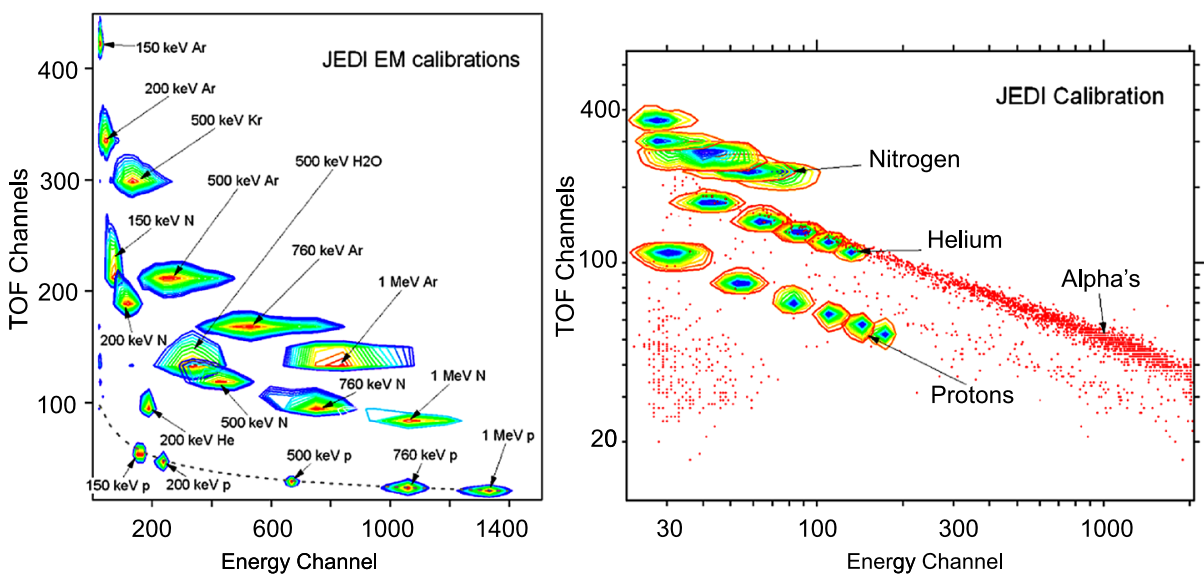

Fig. 32 TOF $\times$ E calibration runs obtained by the high energy ion accelerator at GSFC $(l e f t)$ and the lower energy JHU/APL ion accelerator (right). This figure shows a portion of the broad range of energies detected by JEDI and that for TOF $\times \mathrm{E}$ the elemental species are well separated. On the right panel, the slight shift between the accelerator measurement of helium and the degraded Am241 source measurement of alpha particles is a result of slight differences in the data-number (DN) to energy conversion for different SSD chains; SSD chains used for the two measurements were different. These differences in DN to energy conversion are accounted for in the JEDI calibration matrices
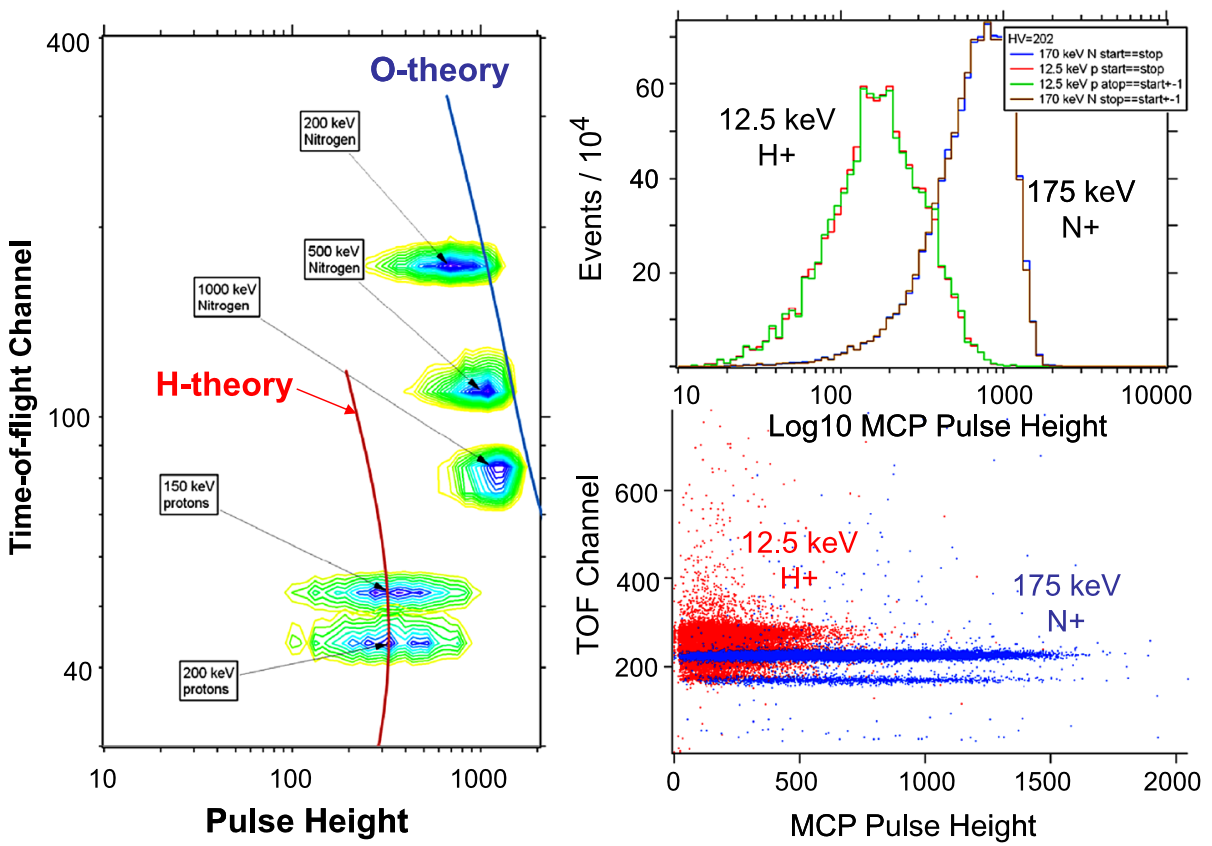

Fig. 33 TOF $\times$ PH calibration runs obtained by the high energy ion accelerator at GSFC (left) and the lower energy JHU/APL ion accelerator $(r i g h t)$. TOF $\times \mathrm{PH}$ measurements do not separate mass species to the degree that TOF $\times \mathrm{E}$ measurements do, but these measurements show that JEDI meets requirements. Clean measurements are obtained by eliminating the measurements in the regions of overlap (e.g. see Fig. 26), at the price of reduced sensitivity 

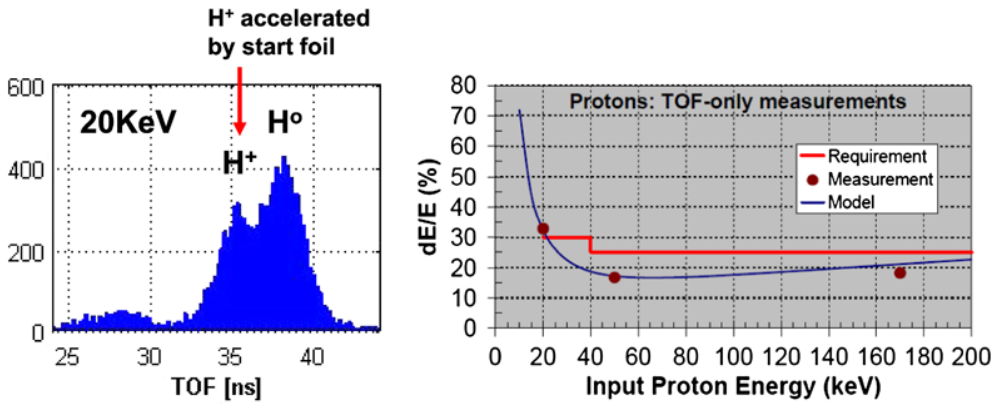

Fig. 34 JHU/APL Ion accelerator calibrations of JEDI showing that the energy resolution requirements are met using the TOF $\times \mathrm{PH}$ measurements at low energies

Fig. 35 GSFC ion accelerator calibrations showing that the efficiency of ion detection with the start and stop foils in JEDI (points) scales roughly with the stopping power $(d E / d X)$ of the ions in matter $(d E / d X$-scaled result are shown with the continuous lines)

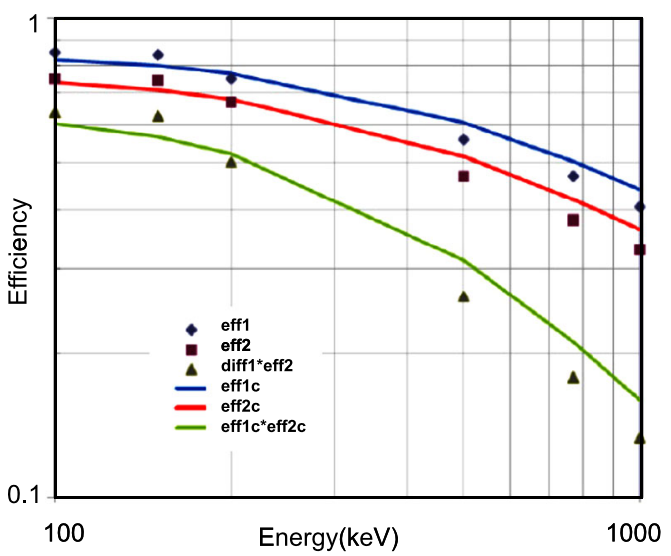

Figure 34 shows by measurement and modeling (right) that JEDI achieves the required ion energy resolution. At these energies it is the TOF $\times \mathrm{PH}$ measurements that are used, and the resolution is determined mostly by time dispersion and scattering (resulting in variable flight distances). At the lowest energies (left) we see that the charge state of the incoming ions are redistributed by interactions within the collimator foil with the result that the energy resolution, crudely defined by the positions of the half amplitudes of the response increases with decreasing energy. The structure within the response (left panel) allows us to deconvolute the response function if needed to robustly achieve the required energy resolution.

Figure 35 demonstrates that the efficiency of secondary electron generation within the start and stop foils (for protons in this particular case) indeed scales roughly with the stopping power, $d E / d X$ (keV/micron). The points are measurements made with an internal capability of JEDI (see Sect. 5.5) and the lines are a model based on the stopping power function.

Figure 36 shows GSFC accelerator runs at selected energies for protons (upper left) and electrons. The proton runs demonstrate the energy resolution capabilities of JEDI SSD measurements and the electron runs show the response of the SSD's to the higher energy electrons for both large and small SSD pixels. Near the top end of the required electron measurements (445 keV against a $500 \mathrm{keV}$ requirement) a fraction of the electrons penetrate the SSD (and some enter the SSD and reflect back out again) resulting in some energy deposition over a broad range of energies below the main peak. This low energy tail will be all 

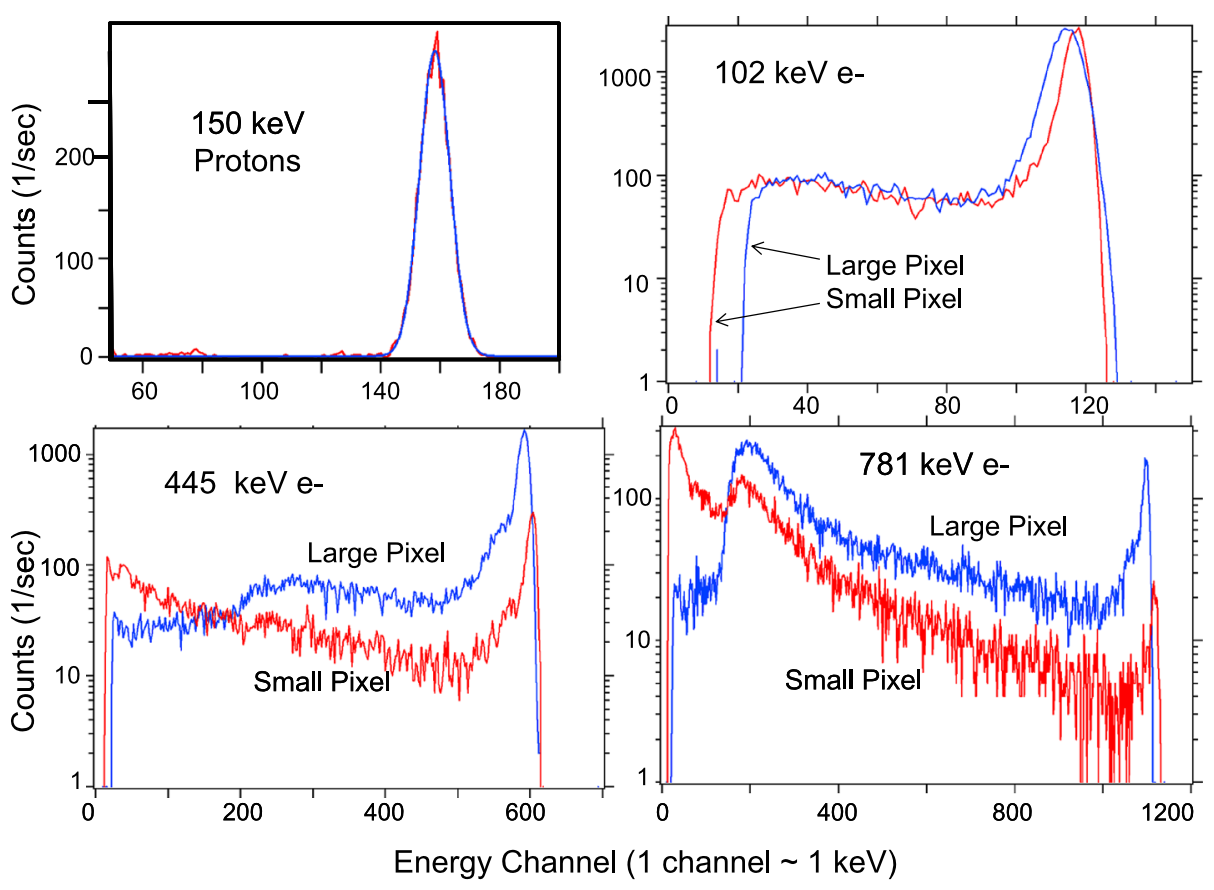

Fig. 36 GSFC accelerator proton (upper left) and electron calibrations of the response of the JEDI SSD's. The proton results (the results displayed here and results not displayed) demonstrate that the SSD's achieve energy resolution requirements, and the electron results show that electrons up to $500 \mathrm{keV}$ are robustly measured. Above $500 \mathrm{keV}$ electron measurements continue to be made but with increasing participation from electrons that completely penetrate the SSDs, given rise to increasing energy deposition below the main peak. The electron panels show that the large and small pixels yield equivalent results for the main peak, but differ in their response to penetrating particles. For measurements up to $500 \mathrm{keV}$, these differences are suppressed for incoming spectra that behave in the normal fashion, with intensities strongly falling with increasing energy

but invisible for normal electron spectra that decrease substantially with increasing energy. For electron energies above those required ( $781 \mathrm{keV}$ in the figure) the lower energy tail becomes a much more prominent feature, and forward modeling will be required to extract the characteristics of the electron spectra at these higher energies. The differences between the small and large pixels at these large energies results from edge affects (a greater influence for the small pixels) and from energy deposition in a guard structure that carries the small pixel signal across the large pixel area (Fig. 23).

The electron measurement shown in Fig. 36, combined with the source radiation source spectra like that shown in Fig. 29 also demonstrate that JEDI achieves the required energy range for the electrons. These same measurements also show that JEDI achieves it electron energy resolution requirements.

Figure 37 shows that JEDI achieves its angle resolution requirements. The top panel shows the $6 \mathrm{TOF} \times \mathrm{PH}$ view directions as they are rotated across an accelerator beam. The bottom panel shows the $6 \mathrm{TOF} \times \mathrm{E}$ view directions as they are similarly rotated across an accelerator beam. The TOF $\times \mathrm{E}$ measurements show a narrower response because the ion portion of the SSD's that participate with the TOF $\times$ E measurements cover only half the backplane (Fig. 23). Note that the determination of the arrival direction of the ions is 
Fig. 37 JHU/APL ion accelerator calibrations showing that the JEDI TOF $\times \mathrm{PH}$ (top) and the TOF $\times \mathrm{E}$ (bottom) measurements achieve the required angular resolutions. The six peaks are from the 6 JEDI look directions as the JEDI instrument is rotated across the beam. The test beam here comprised $\sim 100 \mathrm{keV}$ protons. Because for ions the arrival direction of the ions is determined exclusively using the start signal, subsequent scattering does not affect the angular response (only the efficiency of detection), and so this angular response for ions is very insensitive to ion energy or species

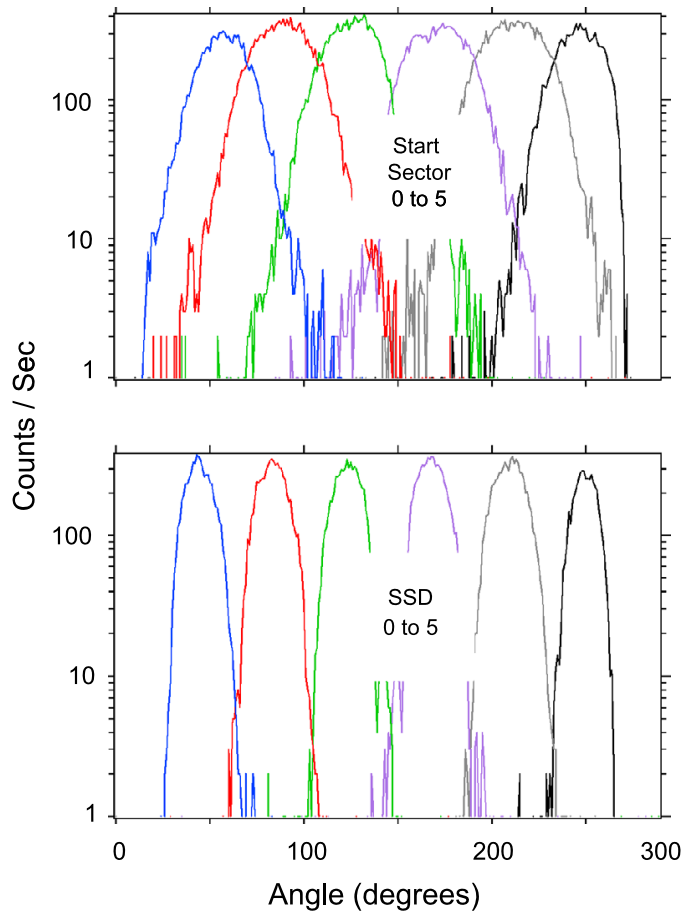

Fig. 38 Measurement of the saturation characteristics of the TOF circuitry as the random input rates become higher. Rates $>10^{6}$ counts/s are retrievable. The label on the right side of the plot is for the two Dead Time (DT) measurements. Each DT count corresponds to $100 \mathrm{~ns}$ of dead time

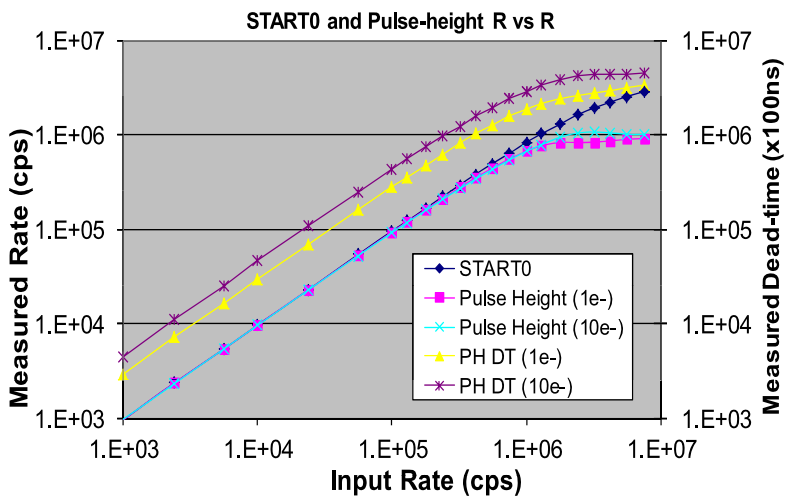

performed at the start position, eliminating the subsequent scattering as a cause for smearing out of the angular response.

Figures 38 and 39 show that the circuitry that measures the TOF (Fig. 38) and energy (Fig. 39) are able to robustly respond to random particle events with event rates as high as $10^{6}$ counts per second. Some roll-off of the response occurs at that rate, but the response remains single valued.

Figure 40 reveals the sensitivity of JEDI ion measurements to strong magnetic fields as a result of the deflection of secondary electron trajectories. For magnetic fields stronger than $10 \mathrm{G}$ (expected right at Jupiter's atmosphere in a few regions) the efficiency is modulated a small amount and the time-of-flight of the very fastest particle $(\sim 1 \mathrm{MeV}$ protons with times of flight about $6 \mathrm{~ns}$ ) can be shifted by $\pm 0.8 \mathrm{~ns}$. Because the energy is measured 
Fig. 39 Measurement of the saturation characteristics of the SSD circuitry as the random imput rates become higher. Rates $>10^{6}$ counts/s are retrievable

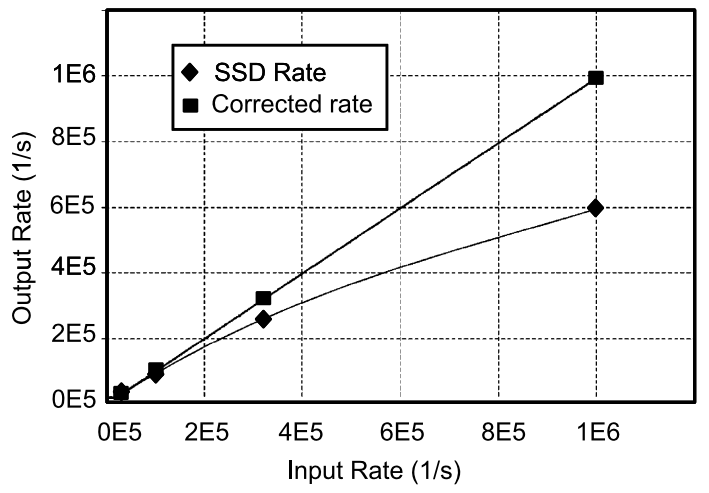

sensitivity of the JEDI TOF measurements when JEDI is placed within a strong magnetic field; $10 \mathrm{G}$ for the case shown here with the direction of the magnetic field oriented to have the worst case effects. Quality measurements can still be made at these levels of magnetic field. Generally the magnetic field within the auroral target regions will be substantially weaker than $10 \mathrm{G}$
4.7 MeV $\alpha$ particle TOF (ns)

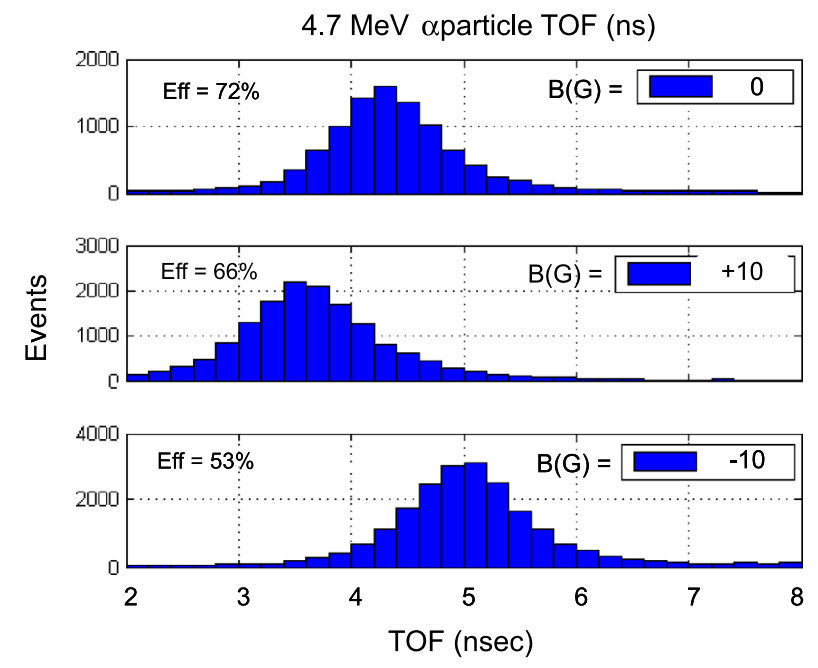

by the SSD not by the TOF function, this shift does not strongly affect the measurement in that it is too small to cause us to misidentify the ion as a helium ion. None-the-less with magnetic fields at $10 \mathrm{nT}$ the highest energy proton measurement will be degraded somewhat. Overwhelmingly with the auroral acceleration regions that Juno will see the magnetic field strengths are expected to be far below the $10 \mathrm{G}$ level. Comprehensive measurements of the JEDI response to magnetic fields up to $16 \mathrm{G}$ in 3 orthogonal directions were obtained which will allow us to decontaminate the JEDI measurements, but unless we are very wrong about the frequency of such contaminations, it is unlikely that we will make the substantial effort to perform such decontaminations.

\subsection{JEDI Data Features}

There are "ghost" features within the JEDI data. Figure 41 is presented here to identify one of the "ghost" features within the JEDI data. The small bump that resides just below the main peak is the result of the primary particle striking an edge of the electrostatic mirror (Figs. 11 and 18), with the generation of secondary electrons that find their way to the MCP. A similar feature from SSD edge effects is apparent in the TOF $\times$ E spectrum shown in 
Fig. 41 TOF spectrum revealing one of the ghost signatures in the JEDI data, the small peak at 85 ns. This peak comes from incoming ions hitting the electrostatic mirror structure and generating secondary electrons that find their way to the MCP

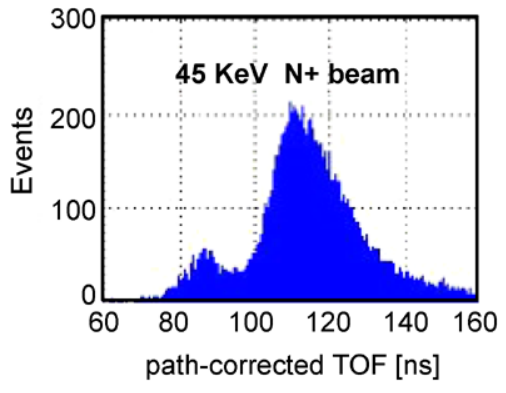

Fig. 30. Other events well below the main track result from penetrations of or scattering off of stainless steel grids that hold the various foils within the sensor.

\subsection{In Flight Calibration Processes and Opportunities}

\subsubsection{Procedures}

The JEDI instruments have an inflight calibration mode (see Sect. 4.6) that injects calibrated pulses into all MCP anode sampling pre-amplifiers and all SSD amplifiers. This process validates the fidelity and stability of the chain of circuits that process the event pulses and injects them into the valid event logic. It also allows for the determination of offsets (again see Sect. 4.6). This mode is actively invoked by command. Our plan is to invoke this mode at least yearly during cruise and at least quarterly during the orbital mission.

For each of the 3 species modes (ion species including $\mathrm{TOF} \times \mathrm{E}$ and $\mathrm{TOF} \times \mathrm{PH}$, electron spectra, and ion spectra) the 3 JEDI sensors comprise 18 different views (6 per instrument). We determine the relative precision between these 18 views by analyzing JEDI data within regions where the particle distributions are roughly time-stationary, homogeneous, and isotropic. Note that for JEDI-90 and JEDI-270, the 12 different views all view the nearly identical directions in space, but at different spin phases. Thus, these sensors can be accurately compared even if the distributions are not precisely isotropic. Differences in the responses of the different views can result from non-uniform gains on the MCP, slightly different channel definitions because of variations between the same components on different instruments or view directions, differences in discrimination levels due to differences in detector noise characteristics, etc. Analysis of the differences in view direction responses can be mitigated by adjustments on the ground to calibration matrices (geometric factors, gain factors, etc.) and adjustments onboard (discrimination levels, MCP bias voltages, new table uploads, etc.). During cruise such inter-comparisons will be performed at least once per year. During orbital operations such inter-comparisons will be performed once per orbit, and on-board mitigations will be uploaded roughly 2 orbits later.

A principle concern is determining and setting the efficiency of detection of secondary electrons coming from the start and stop foils, given the changing gain states of the MCP's over time. There are two features of JEDI that make this process much easier than it has been on heritage instruments. The first feature is that complete detailed pulse height distributions (2048 channels) can be obtained in flight for the start region of the MCP, allowing the detailed response of the start system (start foil, MCP, anodes) to incoming particles (e.g. Fig. 33). To take advantage of this capability, the so-called event data must be telemetered to ground. The complete diagnostic event data (Table 5) can be sent, but generally to preserve telemetry volume a subset of that information is sent. After several hours of 


\section{Measuring "start foil" and "stop foil" efficiencies in flight}

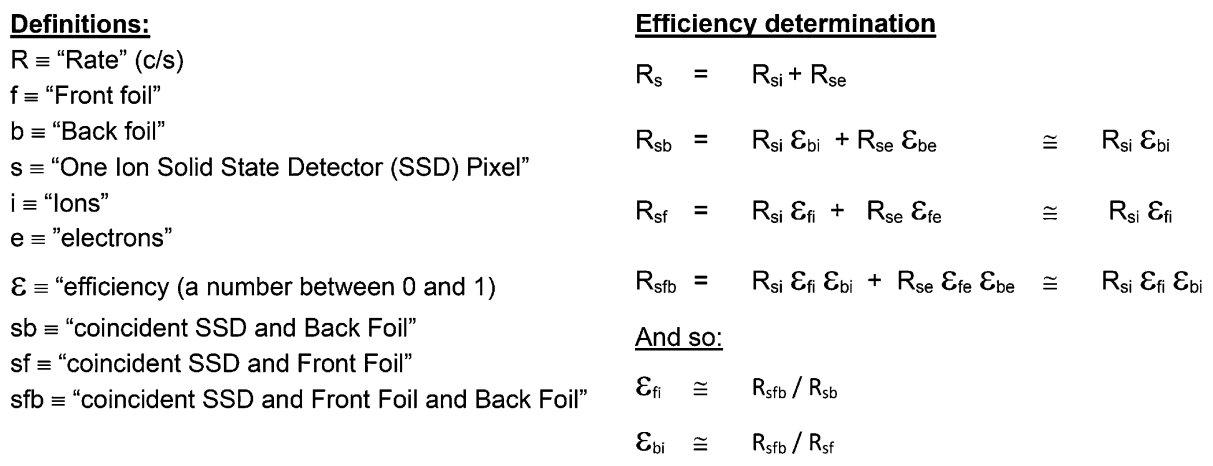

Fig. 42 Expressions showing how the efficiency of secondary electron detection from the start and stop foils are measured directly in flight during periodic calibration

sampling, the individual event data may be sorted according to energy, TOF, Pulse-Height $(\mathrm{PH})$, and look direction, and so the $\mathrm{PH}$ distribution for a "standard candle" energy (e.g. $100 \mathrm{keV}$ protons) for each look direction can be generated and compared with ground distributions and with other distributions in space. Because the pulse height distribution is obtained only for the start pulses, this procedure only diagnoses the evolution of secondary electron detection of the start region, not the stop region. However, the start region is where we expect the more rapid changes in efficiency due to the great flux of particles and UV light onto the start foil than is expected on the stop foil (the geometric factor of just the start foil is a factor of 3-4 greater than the geometric factor of just the stop foil).

The second feature that JEDI contains to determine the efficiencies of secondary electron detection, for both the start and the stop regions, is the ability to count various kinds of coincident events. Table 13 shows that, if we choose to telemeter what are called "diagnostic rates" (generally not sent to the ground in order to save telemetry), there are counters that report coincident SSD-Start counts and coincident SSD-Stop counts. Table 14 shows that we can also report total TOF-SSD counts (non-directional), but also during low counting situations where the JEDI processor is able to process every event, the standard data products can be manipulated to obtain directional TOF-SSD counts. These counters can be combined in the fashion shown in Fig. 42 to obtain the efficiencies of secondary electron generation for both the start regions and the stop regions.

The principal responses to changes in the efficiency of secondary electron generation are: (a) to increase the gain of the MCP by increasing its bias voltage, and/or (b) to modify the $\mathrm{TOF} \times \mathrm{PH}$ look-up tables by adjusting a multiplicative parameter.

Much less of a concern than the MCP gain state is the degradation of SSD performance that results from radiation damage to the outward facing surface of the SSD and the corresponding increase in the thickness of the dead layer above the nominal $50 \mathrm{~nm}$ that prevails on launch. A heritage instrument, the Galileo Energetic Particle instrument (Williams et al. 1994) showed signs of such aging after 2 years in orbit around Jupiter, but with an orbital configuration (equatorial) that likely resulted in much greater damage per year than anticipated with JEDI. Such damage is quite readily detected and quantified by means of the evolution of the positions of species tracks in the TOF $\times \mathrm{E}$ displays like that shown in Fig. 30. For flight data such a display would represent an accumulation over several hours 

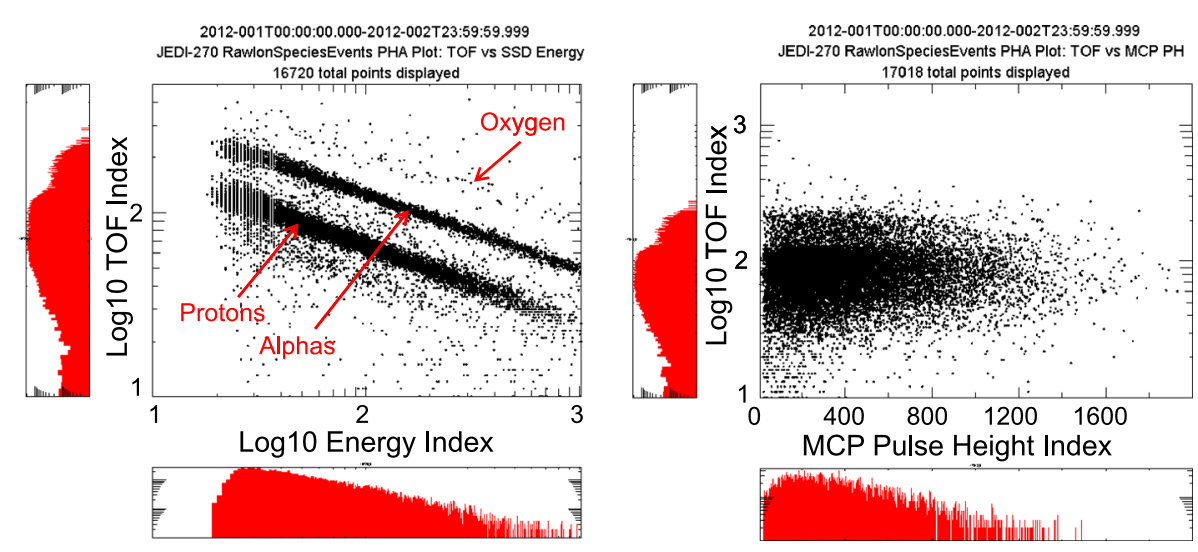

Fig. 43 TOF $\times \mathrm{E}($ left $)$ and TOF $\times$ PH spectra taken in flight by JEDI-270 during an enhancement in the interplanetary ion intensities

of the event data describe above in this section. The mitigation of such degradation is the modification of the TOF $\times \mathrm{E}$ onboard tables in order that the species channels match the species tracks. The energy boundaries of the channels would also have to be modified in the ground calibration tables.

\subsubsection{Opportunities}

In flight calibration will occur within interplanetary space, during an Earth magnetosphere encounter in October of 2014, and within Jupiter's magnetosphere itself. The Earth encounter is a particularly important opportunity to test the JEDI responses in a fairly well know environment and where there are other assets with which to compare the results, most importantly the Van Allen Probes twin spacecraft mission (Mauk et al. 2012) which carries the RBSPICE instruments, a sister instrument to JEDI (Mitchell et al. 2013), but also other instruments that measure energetic charged particles. This opportunity allows the JEDI team to monitor the performances of JEDI in a harsh magnetospheric environment with enough time before the prime Jupiter mission to understand and respond to any unexpected behaviors. A very important set of sequences that we will be running during the Earth encounter is to cycle back and forth between large and small SSD pixels on a spin-by-spin basis to understand very well the differences in the responses to those two configurations. The high voltages of JEDI-90 and JEDI-270 will be operated at Earth out side of $4 \mathrm{RE}$, but the high voltage system of JEDI-A180 will not be operated at Earth because the high voltage system of that unit will not have been fully commissioned (see Sect. 6).

At Jupiter, the very best way to tell just how well JEDI is able to discriminate between various mass species is to simply collect substantial amounts of event data, such as that represented in Figs. 12 left and 43. We have stated, for example, in the discussion of Table 1 that the separation of $\mathrm{O}$ from $\mathrm{S}$ is a goal, not a requirement. There is no better way to characterize the ability of JEDI to make this separation than the collection at Jupiter of TOF $\times \mathrm{E}$ matrices. Calibration of the uniformity of the multiple instrument views within each instrument and between instruments is discussed in Sect. 7. 


\section{JEDI In-Flight Performance in Interplanetary Space}

As of this writing the 3 JEDI instruments have been operating in interplanetary space for close to 1 year, mostly in their "energy modes" with the high voltages turned off. During early commissioning, the high voltages on JEDI-A180, JEDI-90, and JEDI 270 were operated for about 1 day, 12 days, and 16 days, respectively, during commissioning. These high voltages were autonomously turned off by alarms within the instrument generated by a new circuit introduced into the JEDI design that senses small transients on the current outputs from the MCPs (this circuit was not a part of heritage instruments). The cause of these "micro-discharges" has been determined to be high fluxes of solar ultraviolet light entering the sensor. These fluxes of UV light were higher than expected but none-the-less not so high as to overwhelm the TOF system. Extensive ground testing has shown that the instruments are not damaged by the discharges, and subsequent flight JEDI testing has shown that the $\mathrm{HV}$ system continues to operate normally. After uploading new software to give greater flexibility in the JEDI response to the discharges, the 3 JEDI HV systems were re-operated in the April 2013 time frame when the spin axis of the spacecraft is more closely aligned with the sun direction as it will be at Jupiter. JEDI-90 and JEDI-270 were operated at full high voltage for about a week and no HV anomalies occurred. The UV fluences were down one order of magnitude from those that occurred during commissioning because of the better orientation of the spacecraft. These fluences will be another order of magnitude less than observed during this most recent test once Juno get to Jupiter at 5 AU from the sun. JEDIA180 observed a noise which has been diagnosed as being caused by a MCP hot spot that will require a number of weeks to age away once a new low-UV fluence period is identified in the 2014 time frame. It should be noted, however, that the JEDI science requirements and objectives can be fully and robustly achieved with the operation of HV on just the JEDI-90 and JEDI-270 units.

The micro-discharges have been observed in other flight instruments that use MCPs for sensing UV light (Hubble Cosmic Origins Spectrograph FUV: Friedman and Brownsberger 2004; FUSE satellite: documented in the unpublished Sahnow 2003a; and summarized in Sahnow 2003b) causing in-flight high voltage shutdowns; and it now appears to us that this response (called "crackles" in the FUSE report) may be a "feature" of MCP operation when exposed to substantial fluxes of UV light. The high UV fluxes observed by the JEDI sensors occurred at a solar radial distance of about 1.4 AU and with the spacecraft spin axis at an angle ( $\sim 25$ degrees) substantially larger that it will be during science operations at Jupiter ( $<15$ degrees). The UV fluxes that the JEDI sensors will see during the flight operations at Jupiter will be 2 orders of magnitude less than the sensors saw during the original HV commissioning.

While the HV systems were on, the JEDI TOF system operated just as expected. Figure 43 (left) shows a TOF $\times$ E display from JEDI-270 that looks just as it is expected to look on the basis of ground testing. We see clear signatures of $\mathrm{H}$ and $\mathrm{He}$ and a weak signature of $\mathrm{O}$ ions. Figure 43 (right) shows a TOF $\times \mathrm{PH}$ display that shows very healthy pulse-height distributions. There are few heavy ions within the interplanetary environment that would be expected to occupy the upper right quadrant of the display. The MCP gain is somewhat higher than assumed for the TOF $\times \mathrm{PH}$ channelization matrix (Fig. 26), and that mismatch will be adjusted by a simple uploadable multiplicative parameter that rescales the lookup table.

During most of the first year of operation within the interplanetary environment, the JEDI instruments have been in the energy mode and have obtained outstanding measurements. Figure 44 shows the dramatic measurement of a major solar-generated energetic particle 
Fig. 44 JEDI energetic particle measured by the electron SSD's during a major interplanetary particle event. The spinning JEDI was periodically viewing directly at the sun and viewed the X-rays coming from the solar flare event that was involved with the generation of the energetic particle populations

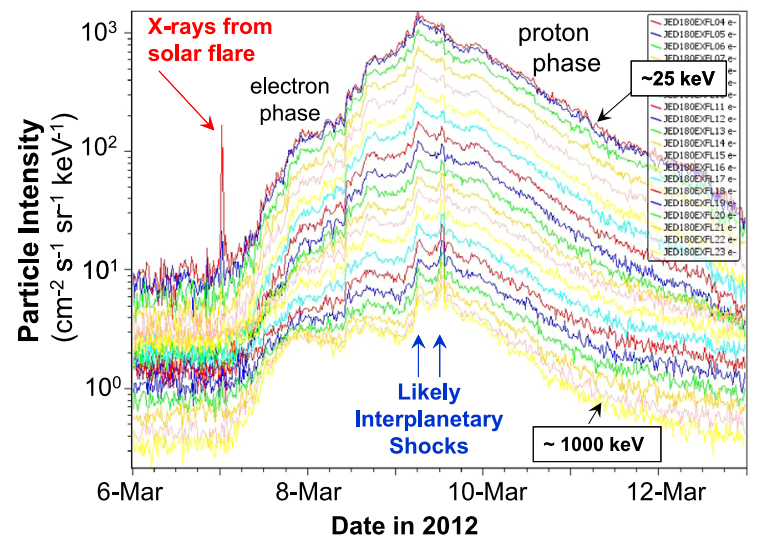

event in early March of 2012. Of substantial interest is that, because of the "non-operational" orientation of the Juno spin axis relative to the sun, the JEDI-A180 field of view actually looked right at the sun once per spin and directly observed the X-rays coming from the solar flare that accompanied the generation of the interplanetary event. This X-ray event was observed by one of the electron SSD's with 2 microns of aluminum flashing. The adjacent ion detector was shadowed by the sun-block in the collimator of the JEDI-A180 instrument (Fig. 2). The v0 ion SSD is protected by $\sim 12$ degree collimator sun block (Fig. 2). The implication of this observation is that during nominal (non-flare) conditions, the SSDs are relatively immune to impingement by the sun into the sensor volume. The rest of the figure shows a population of interplanetary energetic charged particles accelerated by the solar event. Imbedded within the structure of the population are several apparent shock waves that may have participated with the acceleration of the particles.

The charged particles shown in Fig. 44 were measured by the electron SSD's of JEDIA180 instrument. Comparisons between the responses of the electron SSD's and the ion SSD's show that the early part of the event is primarily electrons whereas the later part of the event contains substantial contributions from $>250 \mathrm{keV}$ protons. Figure 45 shows how comparisons between the ion and electron SSD's (bottom) can help separate ions from electrons for SSD-only measurements. The comparison between the total rate from the electron SSD's and that from the ion SSD's shows that the small events within the line plot are purely ions. The energy spectrogram on the top is of the ion feature shown in the bottom panel.

\section{JEDI Operatons}

As a three-sensor instrument, JEDI can operate in a number of different data-taking modes. Also, each of the three sensors can take data at different rates, meaning for instance that A180 may be in a very high data taking mode due to its spatial coverage while JEDI-90 and JEDI-270 are operated at a low rate. The highest rate mode currently used is about 31,000 bits/s, and this includes the total data obtained by all three JEDI sensors. Once the spacecraft is in Jovian orbit, the amount of data that can be transmitted during the downlink will be the critical factor. Due to the finite amount of downlink time per orbit and the demands of each of the instruments and the spacecraft, JEDI has a total allocation of about 1780 Mbits for a representative Jovian orbit (Orbit 10). This does not put constraints on how much data JEDI 
Fig. 45 (Bottom) Electron and ion total SSD detector events during a weak interplanetary event that showed only an ion SSD response, demonstrating that the event included only ions with energies insufficient to get through the flashing on the electron SSD's. (Top) Color spectrogram of this ion-only event
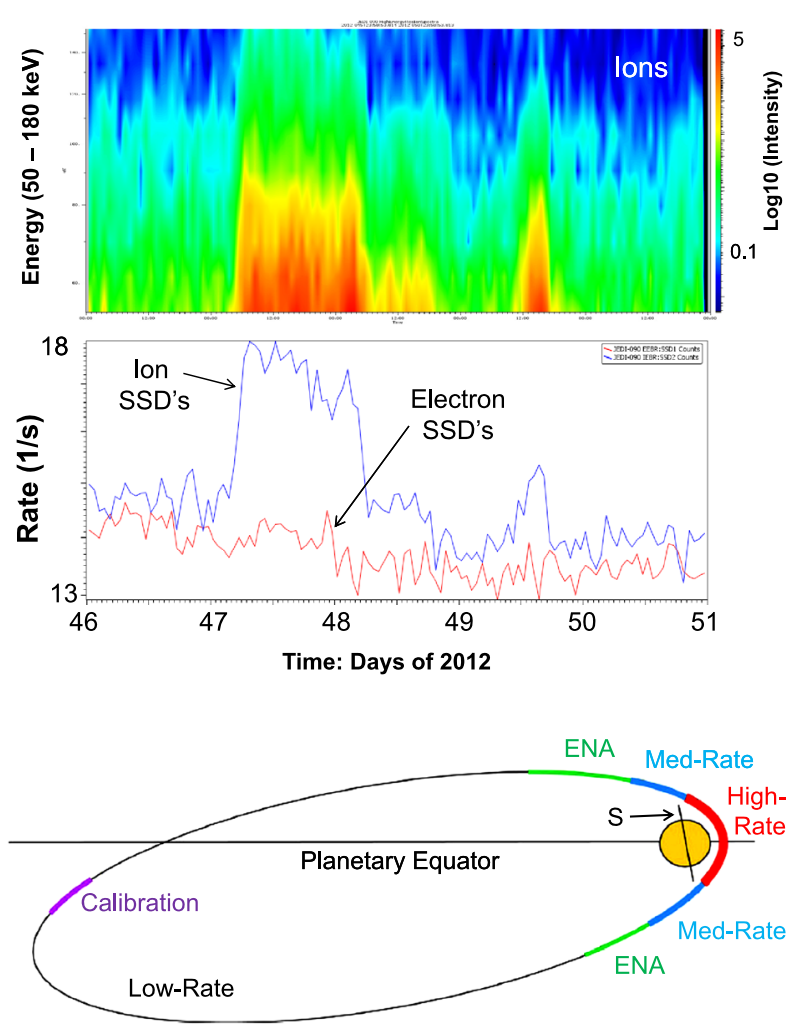

Fig. 46 One orbit of Juno around Jupiter during the science operations with annotations showing the various data taking modes that JEDI will utilize in various regions 


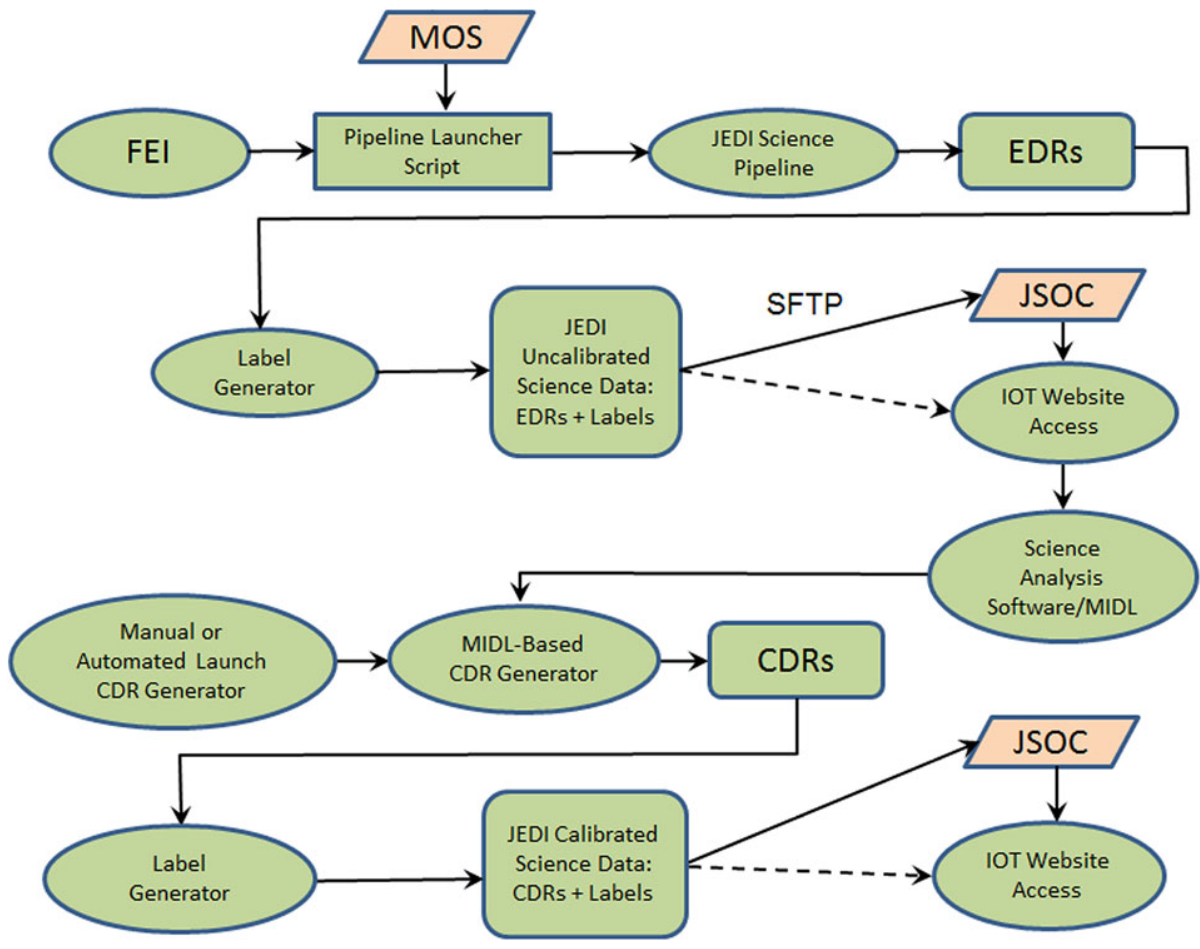

Fig. 47 Diagram of the flow of JEDI data as it is received from the spacecraft, captured, processed, analyzed, and disseminated

mogeneous and roughly isotropic, allowing multiple view directions to be inter-calibrated. This is a slightly higher rate than the low rate used throughout most of the approximately 11 day orbit. It is also used to understand some science issues, such as radial force balance between the fields and particles within Jupiter's unique magnetodisc configuration. To apportion our total data volume, we have divided the data taking into modes in conjunction with the Juno project. In Table 10, we list the specific time periods for a typical orbit when each mode is utilized. These periods are listed relative to perijove (PJ), or the closest approach along the orbit to the planet. In the ENA mode, we are attempting to be at high latitude so that ambient charged particles will not be too competitive with the main ENA signal that may come from Jupiter's exosphere as a result of ions precipitating onto the atmosphere.

\section{JEDI Data Processing, Flow and Archiving}

The flow of JEDI data is illustrated in Fig. 47. Here we describe various aspects of that flow.

\subsection{Obtaining the data from the spacecraft}

Science telemetry data from the JEDI instruments are collected on the Juno spacecraft Solid State Recorder as a collection of files of a fixed but adjustable size: at the time of this writing 
set to 16 kilobytes each. The science data files consist of the raw stream of packets emitted from the instrument, stripped of the headers (CIP and IP/UDP) used for routing on the spacecraft. The "files" are telemetered to the Jet Propulsion Laboratory (JPL) Mission Operations Center (MOC) using the Consultative Committee for Space Data Systems (CCSDS) File Delivery Protocol (CFDP). Engineering telemetry (i.e. status, or housekeeping) data is collected at the MOC as a file of instrument status packets wrapped with standard CCSDS headers.

The individual CFDP files and engineering files are then distributed to the JEDI instrument team (JIOT) via (what is called) the FEI data distribution server process that "pushes" the data including two metadata files (a data transfer label and a Planetary Data SystemPDS - data label) to one of two (science or engineering) FEI client processes running at the JIOT facility via a socket connection (TCP/IP). When new files arrive, the FEI client stores them in a staging location on the JIOT computers and sets a "new data received" marker on the file system. Periodically (currently once an hour), the JIOT pipeline process checks the new data markers and initiates the pipeline process if new data has arrived.

The pipeline moves the newly received data and label files to an archive location and inserts basic file information (first and last packet timestamps and ingest time) into a database. It then initiates the level 2 pipeline. The level 2 pipeline combines and de-commutates the telemetry files into ASCII Comma Separated Values (CSV) files containing 24 hours of data for each packet type and JEDI sensor. The pipeline also produces a PDS label for each file generated.

The first step in science analysis involves loading the level 2 (aka "EDR" for Experiment Data Record) files into what is called the Heliolib energetic particle time series tables using the MIDL (Mission Independent Data Layer) code base at APL. It is during this process that physical calibrations are applied to the raw measured counts and digital levels reported in the telemetry. These Heliolib objects are then used to generate quick look summary plots, to feed the interactive analysis and discovery programs used by the JIOT, and to feed the Level 3 (aka "CDR" for Calibrated Data Record) file generation pipeline.

\subsection{Science Processing at APL}

Science processing involves converting the measurement made aboard the spacecraft to scientifically meaningful data. For the purposes of this discussion, it is useful to consider that some JEDI data may rely exclusively on measurements made by the solid-state detector (SSD) whereas other data will rely on the time-of-flight system. Electrons are measured with SSD's only. The processing of such data on the ground relies on the conversion of counts and accumulation times to physically meaningful intensity (counts per $\mathrm{cm}^{2}-\mathrm{s}-\mathrm{sr}-\mathrm{keV}$ ). To perform such a conversion, it is necessary to correct for any effects the measurement itself had on the particles.

The conversion of count rate to intensity includes the efficiency with which the particles are detected and the geometric factor of the instrument. For example, electrons scatter when they enter materials so it is necessary to capture the fraction of electrons that are detected versus the fraction that initially enter the instrument. Furthermore, the detector is not exposed to the entire sky. There are certain portions of the $4 \pi$ through which particles can reach the SSD. By quantifying these, intensity can be computed. Ions tend to lose a lot of energy in materials. In these cases, it is necessary to estimate the energy loss in the instrument system so that the original "free space" energy of the incoming ion can be approximated.

In addition to the data conversion discussed above, there is efficiency associated with the time-of-flight system. Ions impacting the foil will cause electrons to exit the foil 
in the forward direction. This is the basis of the time-of-flight system. However, depending on particle species and energy, the number of forward- and back-scattered secondary electrons can vary. Therefore there is an efficiency associated with the time-offlight detection that is necessary to include in the conversion from count rate to intensity.

\subsection{Making the Data Useful to Investigators}

Once the data are converted to raw counts per exposure interval (EDR) or count rate or intensity (CDR) they are available to the JEDI team interface called JMIDL. Here the entire mission's data can be accessed by investigators. JMIDL is used primarily for displaying the data, such as in line plots of intensity versus time. But the interface allows investigators to carry out a great deal of research by enabling functions such as averaging data (e.g., creating daily averages) or creating energy spectrograms (e.g., to intercompare different energy channels at the same time). In a parallel process, the mission data are also regularly supplied to the Juno Science Operations Center (JSOC) and to the Planetary Data System (PDS), a node for obtaining data files provided by instrument teams to the public. The PDS data represents the team's best guess at the time of the primarily raw, corrected data.

The group that oversees data flow to the PDS is the Juno DAWG. The DAWG holds regular telecons involving the Juno project and the instrument teams to discuss any issues related to delivering data to the public. These may include for instance the timelines for documentation and data delivery. The DAWG also works to ensure that data are reviewed by scientists for useability.

The JEDI flight software document describes the three JEDI sensors in detail and the modes they will be operated in space. This is an internal team document that describes matters such as the time or energy resolution for a specific mode. This document is the blueprint for the JEDI SIS. The SIS is meant to be a complete description of how the data are obtained and can be used. The SIS is made available to the public through the PDS. It undergoes successive updates that include new calibration and other information obtained while the instrument is taking data.

The guidelines for data processing and publication initially flow from the original mission proposal to NASA. There, specific requirements are stated about each instrument's commitments to the data that will be obtained including the specific cadences, time, species, energy, and pitch angle resolution. The original proposal also dictates the expected science data that will be produced by each instrument team.

Open Access This article is distributed under the terms of the Creative Commons Attribution License which permits any use, distribution, and reproduction in any medium, provided the original author(s) and the source are credited.

\section{Appendix A: JEDI Detailed Specifications}

Provide here are quantitative specifications for each JEDI instrument. The few differences between the different JEDI instruments will be noted where appropriate. 


\section{A.1 JEDI Instrument}

This list refers to each of 3 instruments. For JEDI-A180, two of the small ion SSD pixels are blocked off to act as a "witness detector" for determining the penetrating contamination of the electron measurements (small ion pixel look directions v1 and v3 are blocked). The structure that blocks the small ion pixels also reduces the geometric factor of the large ion pixels by about $20 \%$. Below we provide geometric factors, but these do not include efficiencies (see Fig. 28 and Appendix B). For other measurement characteristics of the JEDI instrument (energy and species responses and resolutions, etc.) see Table 1.

Total Mass:

Operational Power:

Volume (JEDIA-180):

Volume (JEDIA-90/270):

Data Rate:

Data Volume:

Field of View:

Angle resolution:

Total TOF $\times$ PH G:

Pixel TOF $\times$ PH G:

Total Energy G:

Large Pixel Energy G:

Small Pixel Energy G: $\quad 0.000033 \mathrm{~cm}^{2} \mathrm{sr}$ (ion or electron, not both)

Witness-Blocked Ion G: $0.00053 \mathrm{~cm}^{2} \mathrm{sr}$
$6.4 \mathrm{~kg}$ (about $5 \mathrm{~kg}$ of this is box structure for shielding)

$3.1 \mathrm{~W}$

$23.3 \times 16.9 \times 12.8 \mathrm{~cm}$ (box holding instrument; door stowed)

$23.3 \times 15.9 \times 16.1 \mathrm{~cm}$ (box holding instrument; door stowed)

Maximum $16 \mathrm{kbps}$

Minimum per orbit: $275 \mathrm{Mb}$. Typical: $1780 \mathrm{Mb}$

$160 \times 12$ degrees (JEDI-A180 reduced 12 degrees)

(JEDI-A180 v0 ion SSD completely blocked)

$26.7^{\circ} \times 12^{\circ}\left(18^{\circ} \times 12^{\circ}\right.$ achievable by using rotating SSD response with JEDI-90 and JEDI-270)

$0.01 \mathrm{~cm}^{2} \mathrm{sr}$

$0.0017 \mathrm{~cm}^{2} \mathrm{sr}$

$0.004 \mathrm{~cm}^{2} \mathrm{sr}$ (ion or electron, not both)

$0.00067 \mathrm{~cm}^{2} \mathrm{sr}$

$0.0053 \mathrm{~cm}^{2} \mathrm{st}$

\section{A.2 JEDI Viewing}

The coordinate system for each JEDI sensor is shown in Figs. 2 (upper left) and 18. The coordinate system for the spacecraft is shown in Figs. 2 (right) and 15. The coordinate system for each JEDI sensor has its $x$-axis pointing parallel to the center of its $160^{\circ} \times 12^{\circ}$ field-of-view, with the $160^{\circ}$ direction contained within the $x-y$ plane, with the positive $Y$ axis closest to the V5 direction. In Table 11 the central direction of the center of each TOF $\times \mathrm{PH}$, Ion-SSD, and Electron-SSD pixel is given as the angle from the $x$-axis within the $x-y$ plane, with positive angles towards the $-Y$ axis (also toward the direction that has been designated the "v0" direction; we realize that it is unusual to have positive angles towards the $-y$ axis rather than the $+y$ axis). To the right of the each angle in Table 11 is the unit vector of the view direction in the instrument coordinate system. Below we provide the transformation matrix that converts the coordinate system of each of the 3 JEDI sensors into the Juno spacecraft coordinate system. The spacecraft coordinate system is again shown in Figs. 2 and 15. To transform a JEDI coordinate vector ( $\left.V_{\mathrm{JEDI}}\right)$ into the spacecraft frame $\left(V_{\mathrm{SC}}\right)$, one performs the operation: $V_{\mathrm{SC}}=T \cdot V_{\mathrm{JEDI}}$. 
Table 11 JEDI Coordinate view directions; angles/unit vectors

\begin{tabular}{|c|c|c|c|c|c|c|c|c|c|c|c|c|}
\hline & \multicolumn{4}{|c|}{$\mathrm{TOF} \times \mathrm{PH}$} & \multicolumn{4}{|c|}{$\mathrm{TOF} \times \mathrm{E}$ and Ions } & \multicolumn{4}{|c|}{ Electrons } \\
\hline & Degrees & Unit-X & Unit- Y & Unit-Z & Degrees & Unit-X & Unit-Y & Unit-Z & Degrees & Unit-X & Unit-Y & Unit-Z \\
\hline v0 & 66.65 & 0.396 & -0.918 & 0.000 & 72.18 & 0.306 & -0.952 & 0.000 & 61.12 & 0.483 & -0.876 & 0.000 \\
\hline vl & 40.05 & 0.766 & -0.643 & 0.000 & 45.56 & 0.700 & -0.714 & 0.000 & 34.5 & 0.324 & -0.566 & 0.000 \\
\hline v2 & 13.31 & 0.973 & -0.230 & 0.000 & 18.84 & 0.946 & -0.323 & 0.000 & 7.78 & 0.991 & -0.135 & 0.000 \\
\hline v3 & -13.31 & 0.973 & 0.230 & 0.000 & -7.78 & 0.991 & 0.135 & 0.000 & -18.84 & 0.946 & 0.323 & 0.000 \\
\hline $\mathrm{u} 4$ & -40.03 & 0.766 & 0.643 & 0.000 & -34.5 & 0.824 & 0.566 & 0.000 & -45.56 & 0.700 & 0.714 & 0.000 \\
\hline v5 & -66.65 & 0.396 & 0.918 & 0.000 & -61.12 & 0.483 & 0.876 & 0.000 & -72.18 & 0.306 & 0.952 & 0.000 \\
\hline
\end{tabular}

\begin{tabular}{llll}
\hline JEDI_90 Transform: & 0.0242 & -0.9903 & 0.1371 \\
& 0.9848 & 0.0000 & -0.1736 \\
& 0.1720 & 0.1392 & 0.9752 \\
JEDI_270 Transform: & 0.0242 & 0.9903 & 0.1371 \\
& -0.9848 & 0.0000 & 0.1736 \\
& 0.1720 & -0.1392 & 0.9752 \\
JEDI_A180 Transform: & -1 & 0 & 0 \\
& 0 & 0 & -1 \\
& 0 & -1 & 0 \\
\hline
\end{tabular}

\section{A.3 Electronics Box}

Mass: $\quad 4776$ grams

Shield thickness: 100 mils

Shield material: $\quad$ Tungsten-Cooper mixture $\left(\sim 15 \mathrm{~g} / \mathrm{cm}^{3}\right)$

Size: $\quad 15.9 \times 20.7 \times 9.3 \mathrm{~cm}$ (including feet $\&$ EMI shield)

Volume: $\quad 1.06$ Liters (excluding structure, EMI shield, and feet)

Operational Power: 1.53 Watts (Does not include make-up SC heaters)

A.4 Sensor

\section{A.4.1 Sensor Bulk Properties and External Structure}

Mass:

4115 grams

Volume:

$196.35 \mathrm{~cm}^{3}$

Shield thickness: $\quad$ See Sect. A.5

Shield material See Sect. A.5

Radiation tolerance; All parts $>100 \mathrm{Krad}$ (EEPROM in radpack)

Operational Power 0.33 Watts

\section{A.4.2 Collimator (Structure, Distances, Thicknesses, Hole Sizes, etc.)}

The collimator (Fig. 21) is made of Tungsten-Copper with a density of about $15 \mathrm{~g} / \mathrm{cm}^{3}$. Note that JEDI A180 has look direction v0-ion blocked in collimator (12 degrees of blockage). This look direction is the closest to looking along the spin axis (direction of high gain antenna) and is blocked to prevent light contamination. In the list below, "radius" is the radial 
position of the outer surface of the blade from the center of the cylindrical TOF chamber. The holes in the blades are linearly graduated according to that radial position. Finally, all lines that pass through the centers of the holes as one moves from blade to blade also pass through the single point that is the symmetry position of the cylindrical TOF chamber (in cylindrical coordinates the symmetry point not only in " $r$ " but in " $\mathrm{z}$ " as well).

Outer blade (1): $\quad$ Radius: $4.991 \mathrm{~cm}$; Thickness: $0.101 \mathrm{~cm}$; Holes: $2.79 \mathrm{~mm}$ dia. Blade 2: $\quad$ Radius: $4.597 \mathrm{~cm}$; Thickness: $0.050 \mathrm{~cm}$; Holes: $2.57 \mathrm{~mm}$ dia. Blade 3 (with foil): Radius: $4.166 \mathrm{~cm}$; Thickness: $0.051 \mathrm{~cm}$; Holes: $2.64 \mathrm{~mm}$ dia. Blade 4: $\quad$ Radius: $3.632 \mathrm{~cm}$; Thickness: $0.051 \mathrm{~cm}$; Holes: $2.03 \mathrm{~mm}$ dia. Blade 5: $\quad$ Radius: $3.251 \mathrm{~cm}$; Thickness: $0.051 \mathrm{~cm}$; Holes: $1.83 \mathrm{~mm}$ dia.

\section{A.4.3 Foils (See Fig. 20)}

In the list below, "radius" is the radial position of the foil from the center of the foil from the cylindrical TOF chamber

Collimator foil: Radius: $4.166 \mathrm{~cm} ; 350 \AA \mathrm{Al}$

Collimator grid: 70 line/inch Stainless Steel, $90 \%$ transmission normal incidence

Start foil: $\quad$ Radius: $3.0 \mathrm{~cm}$; $50 \AA$ Carbon, $350 \AA$ polyimide, $50 \AA$ Carbon

Stop foil: $\quad$ Radius: $3.0 \mathrm{~cm}$; Start foil $+200 \AA \mathrm{Al}$

Grids: $\quad 70$ lines/inch $\mathrm{Ni}, 90 \%$ transmission normal incidence

\section{A.4.4 TOF Chamber (See Fig. 20)}

In the list below, "radius" is the radial position of the outer surface of the chamber from the center of the chamber.

Entrance window: Height: $0.6 \mathrm{~cm}$

Radius of window: $3.0 \mathrm{~cm}$

Chamber diameter: $6.0 \mathrm{~cm}$

\section{A.4.5 MCP Sensors (See Fig. 19)}

The MCP comprises 2 circular Burle MCP plates in the chevron configuration with a small gap between them and operated with high voltages between 1900 and 2400 volts over the life-time of the plates.

Diameters: $\quad 5 \mathrm{~cm} ; 4 \mathrm{~cm}$ quality diameter

Plate thickness: $0.06 \mathrm{~cm}$

Pore size: $\quad 10$ microns

Pore pitch: $12 \mathrm{um}$

Bias angle: $\quad 12^{\circ}$

Stack gain: $\quad$ Several $\times 10^{6}$

\section{A.4.6 SSDs and SSD Arrays (See Figs. 19 and 23)}

The Each JEDI comprises 6 SSD's with 4 pixels each manufactured by Canberra. The electrical signal from the small pixels is measured using a "channel" that runs across the face of the large pixel (Fig. 23) that can contribute a small degraded contaminating signal to the large pixel response. The SSD is mounted on printed circuit board (all but hidden in Fig. 23), 


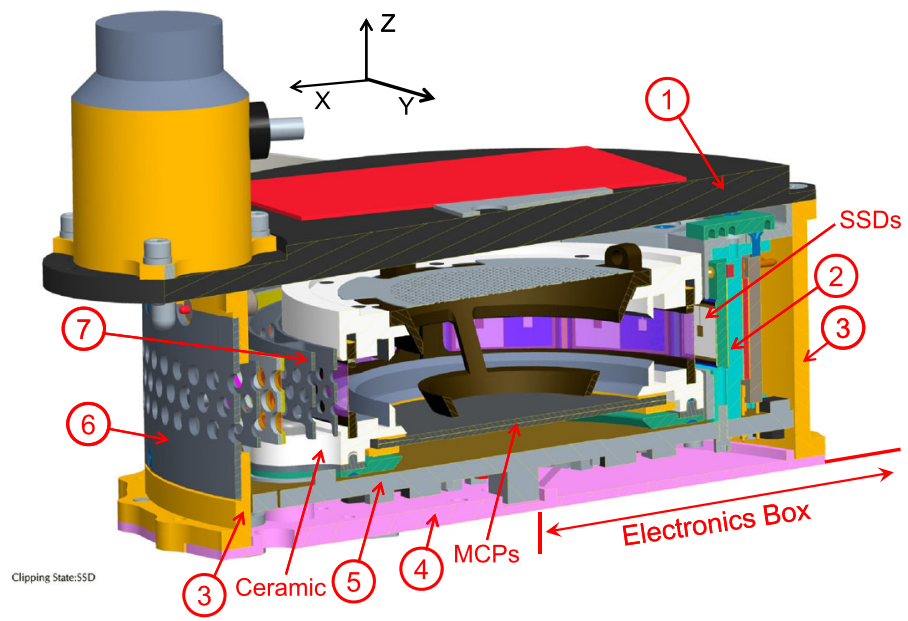

Fig. 48 Cross Section of one of the JEDI sensor showing the shielding configuration for both the SSD and the MCP sensors. See the text in Appendix A.5 for a discussion of this figure

which in turn is mounted on the Tungsten-Cooper "hangers." For JEDI-A180, the small ion pixels for look directions v1 and v3 are blocked by small metal plates so that those pixels act as witness detectors for determining penetrating radiation (see Sect. 3.3). Those witness blockages also cover about $20 \%$ of the large pixels as well. In the list below, the word "radius" refers to the radial position with respect to the center of the TOF chamber.

Radius of SSD array: Surface facing particles: $3.38 \mathrm{~cm}$

Thickess: $\quad 500$ microns $(0.05 \mathrm{~cm})$

Deadlayers: $\quad$ Roughly $50 \mathrm{~nm}$ as measured by JEDI team

Size: $\quad 14.7 \times 8.5 \mathrm{~mm}$

Large pixel size: $\quad 6.20 \times 6.45 \mathrm{~mm}$; Area $=40 \mathrm{~mm}^{2}$

Small pixel size: $\quad 1.28 \times 1.55 \mathrm{~mm}$; Area $=2 \mathrm{~mm}^{2}$

Electron pixel flashing: 2 microns $\mathrm{Al}$

Channel size: $\quad 0.2 \times 2.3 \mathrm{~mm}$; Area $=0.46 \mathrm{~mm}^{2}$

Witness blockage: $\quad$ Thickness $=0.64 \mathrm{~mm}$; material $=\mathrm{Ti}$

\section{A.5 Sensor Shielding}

Figure 48 shows a cross section of the JEDI sensor with the various elements labeled that provide the shielding for the SSD and for the MCP. This cut through the sensor is rotated somewhat away from the symmetry axis of the sensor (contrary to what is shown in Fig. 18). There is a menagerie of structures that provide partial protection to these sensors, but labeled here are the primary structures that are specifically designed to provide shielding. Item (1) is 200 mils of WCu providing shielding of 7.5 to $8 \mathrm{gm} / \mathrm{cm}^{2}$. The cylindrical can (Item (3)) is 100 mils of WCu providing shielding of 3.75 to $4 \mathrm{gm} / \mathrm{cm}^{2}$. The SSD hangers (Item (2)) provide and additional 100 mils of $\mathrm{WCu}$, or 3.75 to $4 \mathrm{gm} / \mathrm{cm}^{2}$ shielding to the backs of the SSD's for a total to the backs of the SSD of 7.5 to $8 \mathrm{gm} / \mathrm{cm}^{2}$. A combination of Items (4) and (5) (100 mils WCu each) and the very well shielded electronics box, provides a minimum of 200 mils of $\mathrm{WCu}$ or 7.5 to $8 \mathrm{gm} / \mathrm{cm}^{2}$, to the bottom of the sensor can. From the direction of the sensor field of view to the left, those particles that do not enter through the sensor 
Table 12 Electron (or Ion) Energy Basic Rate Counters

\begin{tabular}{|c|c|}
\hline Name & Description \\
\hline SSD 0 & Counts events above SSD energy threshold \\
\hline \multicolumn{2}{|l|}{$\cdots$} \\
\hline \multicolumn{2}{|l|}{ SSD 5} \\
\hline SSD 0 Dead Time & $\begin{array}{l}\text { Integrates dead-time in each SSD } \\
\text { (100 ns units) }\end{array}$ \\
\hline \multicolumn{2}{|l|}{ SSD 5 Dead Time } \\
\hline State Machine Idle & Event state machine idle time (100 ns units) \\
\hline Multiple Hit Reject & $\begin{array}{l}\text { Counts the number of events rejected due to } \\
\text { simultaneous energy channel events }\end{array}$ \\
\hline Valid Energy Events & Counts valid energy events \\
\hline Valid Events Queued & Counts valid events placed in FIFO \\
\hline Valid Events Processed & Counts valid events processed by software \\
\hline
\end{tabular}

Table 13 Ion Energy Diagnostic Rate Counters

\begin{tabular}{|c|c|}
\hline Name & Description \\
\hline Start0 Anode & Counts events above start 0 anode threshold \\
\hline Start5 Anode & Counts events above start 5 anode threshold \\
\hline Stop0 Anode & Counts events above stop 0 anode threshold \\
\hline Stop5 Anode & Counts events above stop 5 anode threshold \\
\hline TOF Coincidence & Start and stop within 200 ns window \\
\hline $\begin{array}{l}\text { SSD } 0 \text { with Start } \\
\ldots\end{array}$ & $\begin{array}{l}\text { Counts events above SSD energy threshold } \\
\text { with a corresponding start }\end{array}$ \\
\hline \multicolumn{2}{|l|}{ SSD 5 with Start } \\
\hline $\begin{array}{l}\text { SSD } 0 \text { with Stop } \\
\ldots\end{array}$ & $\begin{array}{l}\text { Counts events above SSD energy threshold } \\
\text { with a corresponding stop }\end{array}$ \\
\hline SSD 5 with Stop & \\
\hline
\end{tabular}

holes are blocked (Items (6) and (7)) with a total of 120 mils of WCu distributed within the 5 blades of the collimator (with thickness of 40,20, 20, 20, and 20 mils of $\mathrm{WCu}$ ) for a total of 4.5 to $4.8 \mathrm{gm} / \mathrm{cm}^{2}$.

\section{A.6 Basic and Diagnostic Rate Tables}

The JEDI hardware counts a variety of pulses from the detectors. In addition to valid particle events, these count foreground, background, and noise events. The valid events seen, as well as the valid events placed in the FIFO are counted. The software also counts the number of events it is able to process. The hardware counters are 24 bits. Every subsector, they are read out and accumulated in 23-bit counters in software. A different set of counters are collected in electron energy, ion energy, and ion species modes. The basic set of counters that are collected in electron or ion energy modes is shown in Table 12. An additional set of diagnostic counters can be collected in ion energy mode and is listed in Table 13. An extensive set of counters are collected in ion species mode are shown in Table 14, and the additional set of diagnostic counters are listed in Table 15. 
Table 14 Ion Species Basic Rate Counters

\begin{tabular}{|c|c|}
\hline Name & Description \\
\hline Start0 Anode & Counts events above start 0 anode threshold \\
\hline Stop0 Anode & Counts events above stop 0 anode threshold \\
\hline TOF Coincidence & Start and stop within 200 ns window \\
\hline Pulse Height & $\begin{array}{l}\text { Counts events above TOF pulse height } \\
\text { threshold }\end{array}$ \\
\hline $\begin{array}{l}\text { Start } 0 \\
\ldots\end{array}$ & $\begin{array}{l}\text { Counts events calculated to be at the given } \\
\text { start position }\end{array}$ \\
\hline Start 5 & \\
\hline $\begin{array}{l}\text { SSD } 0 \\
\ldots \\
\text { SSD } 5\end{array}$ & Counts events above SSD energy threshold \\
\hline $\begin{array}{l}\text { SSD } 0 \text { Dead Time } \\
\ldots\end{array}$ & $\begin{array}{l}\text { Integrates dead-time in each SSD } \\
\text { (100 ns units) }\end{array}$ \\
\hline SSD 5 Dead Time & \\
\hline State Machine Idle & Event state machine idle time (100 ns units) \\
\hline Multiple Hit Reject & $\begin{array}{l}\text { Counts the number of events rejected due } \\
\text { to simultaneous energy channel events }\end{array}$ \\
\hline Valid TOF $\times$ PH Events & Counts valid TOF and pulse height events \\
\hline Valid TOF $\times$ E Events & Counts valid TOF and energy events \\
\hline Valid Events Queued & Counts valid events placed in FIFO \\
\hline Valid Events Processed & Counts valid events processed by software \\
\hline
\end{tabular}

Table 15 Ion Species Diagnostic Rate Counters

\begin{tabular}{ll}
\hline Name & Description \\
\hline $\begin{array}{l}\text { Start5 Anode } \\
\text { Stop5 Anode }\end{array}$ & $\begin{array}{l}\text { Counts events above start5 anode threshold } \\
\text { Counts events above stop5 anode threshold } \\
\text { TOF Valid 1 }\end{array}$ \\
$\ldots$ & $\begin{array}{l}\text { Counts valid event events from each TOF } \\
\text { chip }\end{array}$ \\
$\begin{array}{l}\text { Pulse Height Dead Time } \\
\text { Stop 0 }\end{array}$ & $\begin{array}{l}\text { Integrates dead-time (100 ns units) } \\
\text { Counts events calculated to be at the } \\
\text { given stop position }\end{array}$ \\
Stop 5 & \\
RDT & Counts RDT resets of TOF chips \\
\hline
\end{tabular}

\section{Appendix B: JEDI Channel Definitions}

Tables 16-20 show the characteristics of the measurement channels generated by the JEDI instruments. Shown are electron SSD energy channels, ion SSD energy channels, TOF $\times$ Energy channels, and TOF $\times$ Pulse-Height channels. The energy boundaries and the efficiencies are typical, but the exact numbers for each JEDI unit vary somewhat from the numbers shown in the tables. 
Table 16 JEDI SSD Electron Channels

\begin{tabular}{lccccc}
\hline Species & Channel & $E$ low $(\mathrm{keV})$ & $E$ high $(\mathrm{keV})$ & GF $(\mathrm{LP})$ & Efficiency \\
\hline Electrons & 1 & 18.94 & 20.60 & $6.70 \mathrm{E}-04$ & 0.11 \\
Electrons & 2 & 21.44 & 23.13 & $6.70 \mathrm{E}-04$ & 0.18 \\
Electrons & 3 & 23.99 & 26.56 & $6.70 \mathrm{E}-04$ & 0.26 \\
Electrons & 4 & 27.42 & 31.76 & $6.70 \mathrm{E}-04$ & 0.37 \\
Electrons & 5 & 32.63 & 37.01 & $6.70 \mathrm{E}-04$ & 0.47 \\
Electrons & 6 & 37.89 & 43.17 & $6.70 \mathrm{E}-04$ & 0.54 \\
Electrons & 7 & 44.05 & 51.12 & $6.70 \mathrm{E}-04$ & 0.61 \\
Electrons & 8 & 52.00 & 60.85 & $6.70 \mathrm{E}-04$ & 0.66 \\
Electrons & 9 & 61.73 & 72.32 & $6.70 \mathrm{E}-04$ & 0.69 \\
Electrons & 10 & 73.20 & 86.37 & $6.70 \mathrm{E}-04$ & 0.72 \\
Electrons & 11 & 87.24 & 102.01 & $6.70 \mathrm{E}-04$ & 0.74 \\
Electrons & 12 & 102.88 & 121.72 & $6.70 \mathrm{E}-04$ & 0.77 \\
Electrons & 13 & 122.57 & 144.41 & $6.70 \mathrm{E}-04$ & 0.79 \\
Electrons & 14 & 145.24 & 170.64 & $6.70 \mathrm{E}-04$ & 0.81 \\
Electrons & 15 & 171.45 & 201.67 & $6.70 \mathrm{E}-04$ & 0.83 \\
Electrons & 16 & 202.45 & 237.03 & $6.70 \mathrm{E}-04$ & 0.85 \\
Electrons & 17 & 237.79 & 278.51 & $6.70 \mathrm{E}-04$ & 0.86 \\
Electrons & 18 & 279.24 & 326.30 & $6.70 \mathrm{E}-04$ & 0.87 \\
Electrons & 19 & 327.00 & 381.47 & $6.70 \mathrm{E}-04$ & 0.87 \\
Electrons & 20 & 382.15 & 446.79 & $6.70 \mathrm{E}-04$ & 0.86 \\
Electrons & 21 & 447.45 & 524.32 & $6.70 \mathrm{E}-04$ & 0.86 \\
Electrons & 22 & 524.99 & 618.54 & $6.70 \mathrm{E}-04$ & 0.85 \\
Electrons & 23 & 619.23 & 735.65 & $6.70 \mathrm{E}-04$ & 0.84 \\
\hline & & & & &
\end{tabular}

\begin{tabular}{lllll}
\hline Species Channel & $E$ low $(\mathrm{keV})$ & $E$ high $(\mathrm{keV})$ & $\mathrm{GF}(\mathrm{LP})$ & Efficiency
\end{tabular} Channels

\begin{tabular}{lrrrrr} 
Ions & 1 & 44.26 & 46.43 & $6.70 \mathrm{E}-04$ & 0.95 \\
Ions & 2 & 47.51 & 49.62 & $6.70 \mathrm{E}-04$ & 0.98 \\
Ions & 3 & 50.66 & 53.74 & $6.70 \mathrm{E}-04$ & 0.99 \\
Ions & 4 & 54.75 & 59.70 & $6.70 \mathrm{E}-04$ & 1.01 \\
Ions & 5 & 60.68 & 65.48 & $6.70 \mathrm{E}-04$ & 1.02 \\
Ions & 6 & 66.43 & 72.04 & $6.70 \mathrm{E}-04$ & 1.03 \\
Ions & 7 & 72.96 & 80.24 & $6.70 \mathrm{E}-04$ & 1.03 \\
Ions & 8 & 81.14 & 90.02 & $6.70 \mathrm{E}-04$ & 1.03 \\
Ions & 9 & 90.89 & 101.29 & $6.70 \mathrm{E}-04$ & 1.03 \\
Ions & 10 & 102.14 & 114.83 & $6.70 \mathrm{E}-04$ & 1.02 \\
Ions & 11 & 115.67 & 129.74 & $6.70 \mathrm{E}-04$ & 1.01 \\
Ions & 12 & 130.56 & 148.37 & $6.70 \mathrm{E}-04$ & 1.01 \\
Ions & 13 & 149.17 & 169.77 & $6.70 \mathrm{E}-04$ & 1.00 \\
Ions & 14 & 170.56 & 194.59 & $6.70 \mathrm{E}-04$ & 0.99 \\
Ions & 15 & 195.35 & 224.16 & $6.70 \mathrm{E}-04$ & 0.99 \\
Ions & 16 & 224.91 & 258.25 & $6.70 \mathrm{E}-04$ & 0.98 \\
Ions & 17 & 258.98 & 298.79 & $6.70 \mathrm{E}-04$ & 0.98 \\
Ions & 18 & 299.51 & 346.19 & $6.70 \mathrm{E}-04$ & 0.98 \\
Ions & 19 & 346.89 & 401.59 & $6.70 \mathrm{E}-04$ & 0.98 \\
Ions & 20 & 402.27 & 467.60 & $6.70 \mathrm{E}-04$ & 0.99 \\
Ions & 21 & 468.28 & 545.75 & $6.70 \mathrm{E}-04$ & 0.99 \\
Ions & 22 & 546.42 & 639.50 & $6.70 \mathrm{E}-04$ & 1.00 \\
Ions & 23 & 640.18 & 753.89 & $6.70 \mathrm{E}-04$ & 1.00 \\
\hline & & & & &
\end{tabular}


Table 18 JEDI TOF $\times$ E Proton Channels

Table 19 JEDI TOF x E heavy Ion Channels

\begin{tabular}{|c|c|c|c|c|c|}
\hline Species & Channel & $E$ low $(\mathrm{keV})$ & $E$ high (keV) & GF (LP) & Efficiency \\
\hline Protons & 1 & 37.81 & 40.13 & $6.70 \mathrm{E}-04$ & 0.47 \\
\hline Protons & 2 & 41.26 & 44.56 & $6.70 \mathrm{E}-04$ & 0.51 \\
\hline Protons & 3 & 45.63 & 49.81 & $6.70 \mathrm{E}-04$ & 0.55 \\
\hline Protons & 4 & 50.83 & 54.83 & $6.70 \mathrm{E}-04$ & 0.58 \\
\hline Protons & 5 & 55.81 & 62.52 & $6.70 \mathrm{E}-04$ & 0.59 \\
\hline Protons & 6 & 63.46 & 70.83 & $6.70 \mathrm{E}-04$ & 0.60 \\
\hline Protons & 7 & 71.73 & 80.62 & $6.70 \mathrm{E}-04$ & 0.61 \\
\hline Protons & 8 & 81.49 & 91.81 & $6.70 \mathrm{E}-04$ & 0.61 \\
\hline Protons & 9 & 92.66 & 105.99 & $6.70 \mathrm{E}-04$ & 0.61 \\
\hline Protons & 10 & 106.81 & 122.96 & $6.70 \mathrm{E}-04$ & 0.61 \\
\hline Protons & 11 & 123.76 & 143.33 & $6.70 \mathrm{E}-04$ & 0.59 \\
\hline Protons & 12 & 144.10 & 167.62 & $6.70 \mathrm{E}-04$ & 0.58 \\
\hline Protons & 13 & 168.37 & 196.29 & $6.70 \mathrm{E}-04$ & 0.55 \\
\hline Protons & 14 & 197.01 & 231.11 & $6.70 \mathrm{E}-04$ & 0.52 \\
\hline Protons & 15 & 231.80 & 272.96 & $6.70 \mathrm{E}-04$ & 0.49 \\
\hline Protons & 16 & 273.63 & 322.65 & $6.70 \mathrm{E}-04$ & 0.45 \\
\hline Protons & 17 & 323.30 & 381.73 & $6.70 \mathrm{E}-04$ & 0.41 \\
\hline Protons & 18 & 382.37 & 453.31 & $6.70 \mathrm{E}-04$ & 0.37 \\
\hline Protons & 19 & 453.94 & 541.90 & $6.70 \mathrm{E}-04$ & 0.32 \\
\hline Protons & 20 & 542.53 & 653.01 & $6.70 \mathrm{E}-04$ & 0.28 \\
\hline Protons & 21 & 653.66 & 796.91 & $6.70 \mathrm{E}-04$ & 0.23 \\
\hline Protons & 22 & 797.59 & 984.78 & $6.70 \mathrm{E}-04$ & 0.19 \\
\hline Protons & 23 & 985.50 & 2016.24 & $6.70 \mathrm{E}-04$ & 0.11 \\
\hline
\end{tabular}

Species Channel $E$ low (keV) $E$ high (keV) GF (LP) Efficiency

\begin{tabular}{lrrrrl}
\hline Helium & 0 & 53.64 & 125.88 & $6.70 \mathrm{E}-04$ & 0.49 \\
Helium & 1 & 126.95 & 377.04 & $6.70 \mathrm{E}-04$ & 0.76 \\
Helium & 2 & 377.75 & 2079.18 & $6.70 \mathrm{E}-04$ & 0.94 \\
O+S & 3 & 135.62 & 162.75 & $6.70 \mathrm{E}-04$ & 0.39 \\
O+S & 4 & 164.32 & 218.15 & $6.70 \mathrm{E}-04$ & 0.56 \\
O+S & 5 & 219.45 & 322.30 & $6.70 \mathrm{E}-04$ & 0.75 \\
Oxygen & 6 & 323.37 & 512.36 & $6.70 \mathrm{E}-04$ & 0.85 \\
Oxygen & 7 & 513.22 & 868.28 & $6.70 \mathrm{E}-04$ & 0.92 \\
Oxygen & 8 & 869.05 & 2470.13 & $6.70 \mathrm{E}-04$ & 0.95 \\
Sulfur & 9 & 424.29 & 660.32 & $6.70 \mathrm{E}-04$ & 0.86 \\
Sulfur & 10 & 661.35 & 1073.78 & $6.70 \mathrm{E}-04$ & 0.92 \\
Sulfur & 11 & 1074.64 & 2760.32 & $6.70 \mathrm{E}-04$ & 0.95 \\
Oxygen & 12 & 2498.29 & 5389.95 & $6.70 \mathrm{E}-04$ & 1.00 \\
Oxygen & 13 & 5416.60 & 8434.53 & $6.70 \mathrm{E}-04$ & 1.00 \\
Oxygen & 14 & 8460.46 & 10651.06 & $6.70 \mathrm{E}-04$ & 1.00 \\
Sulfur & 15 & 2789.12 & 5766.68 & $6.70 \mathrm{E}-04$ & 0.96 \\
Sulfur & 16 & 5793.53 & 8844.86 & $6.70 \mathrm{E}-04$ & 1.00 \\
Sulfur & 17 & 8871.19 & 11049.28 & $6.70 \mathrm{E}-04$ & 1.00 \\
\hline & & & & &
\end{tabular}


Table 20 JEDI TOF $\times$ PH Channels

\begin{tabular}{lccccl}
\hline Species & Channel & $E$ low $(\mathrm{keV})$ & $E$ high $(\mathrm{keV})$ & GF & Efficiency \\
\hline Protons & 1 & 15.07 & 17.44 & $1.70 \mathrm{E}-03$ & 0.08 \\
Protons & 2 & 17.55 & 20.44 & $1.70 \mathrm{E}-03$ & 0.13 \\
Protons & 3 & 20.58 & 23.86 & $1.70 \mathrm{E}-03$ & 0.20 \\
Protons & 4 & 24.05 & 28.30 & $1.70 \mathrm{E}-03$ & 0.28 \\
Protons & 5 & 28.57 & 33.50 & $1.70 \mathrm{E}-03$ & 0.37 \\
Protons & 6 & 33.86 & 39.76 & $1.70 \mathrm{E}-03$ & 0.45 \\
Protons & 7 & 40.25 & 47.81 & $1.70 \mathrm{E}-03$ & 0.52 \\
Protons & 8 & 48.49 & 58.29 & $1.70 \mathrm{E}-03$ & 0.58 \\
Protons & 9 & 59.27 & 69.47 & $1.70 \mathrm{E}-03$ & 0.60 \\
Protons & 10 & 70.79 & 84.90 & $1.70 \mathrm{E}-03$ & 0.61 \\
Protons & 11 & 86.76 & 104.52 & $1.70 \mathrm{E}-03$ & 0.61 \\
Protons & 12 & 107.17 & 129.31 & $1.70 \mathrm{E}-03$ & 0.60 \\
Protons & 13 & 133.09 & 160.13 & $1.70 \mathrm{E}-03$ & 0.59 \\
Protons & 14 & 165.51 & 197.42 & $1.70 \mathrm{E}-03$ & 0.55 \\
Protons & 15 & 205.00 & 240.54 & $1.70 \mathrm{E}-03$ & 0.51 \\
Heavy & 0 & 51.14 & 70.14 & $1.70 \mathrm{E}-03$ & 0.02 \\
Heavy & 1 & 70.27 & 85.23 & $1.70 \mathrm{E}-03$ & 0.06 \\
Heavy & 2 & 85.47 & 111.35 & $1.70 \mathrm{E}-03$ & 0.14 \\
Heavy & 3 & 111.79 & 135.68 & $1.70 \mathrm{E}-03$ & 0.26 \\
Heavy & 4 & 136.37 & 161.69 & $1.70 \mathrm{E}-03$ & 0.39 \\
Heavy & 5 & 162.66 & 199.49 & $1.70 \mathrm{E}-03$ & 0.52 \\
Heavy & 6 & 200.94 & 240.41 & $1.70 \mathrm{E}-03$ & 0.65 \\
Heavy & 7 & 242.44 & 287.62 & $1.70 \mathrm{E}-03$ & 0.75 \\
\hline & & & & &
\end{tabular}

\section{References}

R.L. Arnoldy, Review of auroral particle precipitation, in Physics of Auroral Arc Formation, Geophys. Monogr. Ser, vol. 25, ed. by S.-I. Akasofu, J.R. Kan (AGU, Washington, 1981), p. 56. doi:10.1029/ GM025

F. Bagenal et al., Magnetospheric science objectives of the Juno Mission. Space Sci. Rev. (2013, this issue).

S. Bolton et al., The Juno mission overview. (2013, this issue)

J.T. Clarke et al., Ultraviolet emissions for the magnetic footprints of Io, Ganymede, and Europa on Jupiter. Nature 415, 997 (2002). doi:doi:10.1038/415997a

J.E.P. Connerney, M.H. Acuna, N.F. Ness, Voyager 1 assessment of Jupiter's planetary magnetic field. J. Geophys. Res. 87, 3623 (1992). doi:10.1029/JA087iA05p03623

R.F. Elsner et al., Simultaneous Chandra X ray, Hubble space telescope ultraviolet, and Ulysses radio observations of Jupiter's aurora. J. Geophys. Res. 11(0), A01207 (2005). doi:10.1029/2004JA010717

S.D. Friedman, K. Brownsberger, High voltage current transients in the cosmic origins spectrograph FUV detector. COS technical report 2004-02 (v1) (2004). www.stsci/hst/cos/documents/

H. Funsten et al., Helium, oxygen, proton, and electron (HOPE) mass spectrometer for the radiation belt storm probes mission. Space Sci. Rev. (2012). doi:10.1007/s11214-013-9968-7

H.B. Garrett, I. Jun, J.M. Ratliff, R.W. Evans, G.A. Clough, R.W. McEntire, Galileo interim radiation electron model. JPL publ. 03-006, 72 pp, Jet Propulsion Lab., California Inst. of Technol, Pasadena, Calif (2003)

J.R. Hayes, The scalable configurable instrument processor, in Military and Aerospace Programmable Logic Devices Conference (MAPLD), (2005)

J. Hölzl, K. Jacobi, Surf. Sci. 14(2), 351 (1969). doi:10.1016/0039-6028(69)90084-3

A.P. Ingersoll et al., Imaging Jupiter's aurora at visible wavelengths. Icarus 135, 251 (1998)

A. Keiling et al., Auroral Phenomenology and Magnetospheric Processes: Earth and Other Processes. Geophysical Monograph, vol. 197 (American Geophysical Union, Washington, 2012). doi:1029/GM197 
S.M. Krimigis et al., Magnetosphere imaging instrument (MIMI) on the Cassini mission to Saturn/Titan. Space Sci. Rev. 114(1-4), 233-329 (2004). doi:10.1007/s11214-004-1410-8

B.H. Mauk, J.T. Clarke, D. Grodent, J.H. Waite Jr., C.P. Paranicas, D.J. Williams, Transient aurora on Jupiter from injections of magnetospheric electrons. Nature 415, 1003-1005 (2002). doi:10.1038/4151003a

B.H. Mauk, D.G. Mitchell, R.W. McEntire, C.P. Paranicas, E.C. Roelof, D.J. Williams, S.M. Krimigis, A. Lagg, Energetic ion characteristics and neutral gas interactions in Jupiter's magnetosphere. J. Geophys. Res. 109, A09S12 (2004). doi:10.1029/2003JA010270

B.H. Mauk, J. Saur, Equatorial electron beams and auroral structuring at Jupiter. J. Geophys. Res. 112, A10221 (2007). doi:10.1029/2007JA012370

B.H. Mauk, N.J. Fox, Electron radiation belts of the solar system. J. Geophys. Res. 115, A12220 (2010). doi:10.1029/2010JA015660

B.H. Mauk, F. Bagenal, Comparative auroral physics, in Auroral Phenomenology and Magnetospheric Processes: Earth and Other Processes, ed. by A. Keiling et al. Geophysical Monograph, vol. 197 (American Geophysical Union, Washington, 2012). doi:10.1029/2011GM001192

B.H. Mauk, N.J. Fox, S.G. Kanekal, R.L. Kessel, D.G. Sibeck, A. Ukhorskiy, Science objectives and rationale for the Radiation Belt Storm Probes mission. Space Sci. Rev. (2012). doi:10.1007/s11214-012-9908-y

R.E. McNutt et al., The Pluto energetic particle spectrometer on the New Horizons Mission. Space Sci. Rev. 140(1-4), P315 (2008). doi:10.1007/s11214-0089436-y

R.W. McEntire, E.P. Keath, D.E. Fort, A.T.Y. Lui, S.M. Krimigis, The medium energy particle analyzer on the AMPTEE CCE spacecraft, in Geoscience and Remote Sensing, IEEE Transactions on Geoscience and Remote Sensing (GE-23), vol. 3, (1985), p. 230. doi:10.1109/TGRS.1985.289518

D.G. Mitchell, P.C. Brandt, E.C. Roelof, D.C. Hamilton, K.C. Retterer, S. Mende, Global imaging of O ${ }^{+}$ from IMAGE/HENA. Space Sci. Rev. 109, 63-75 (2003). doi:10.1023/B:SPAC.0000007513.55076.00

D.G. Mitchell et al., Radiation Belt Storm Probes ion composition experiment. Space Sci. Rev. (2013). doi:10.1007/s11214-013-9965-X

N.P. Paschalidis, A family of analog and mixed signal VLSI ASICs for NASA science missions. Acta Astronaut. 59, 974-980 (2006). doi:10.1016/j.actaastro.2005.07.044

N.P. Paschalidis et al., A CMOS time of flight system on a chip for spacecraft instrumentation. IEEE Trans. Nucl. Sci. 49, 1156-1163 (2002). doi:10.1016/j.actaastro.2005.07.044

D. Sahnow, FUSE crackles/current transients. Unpublished report (2003a)

D. Sahnow, The FUSE detectors: on orbit use and lessons learned. Proc. SPIE 4854, 610 (2003b). doi: $10.1117 / 12.459777$

D.J. Williams, R.W. McEntire, S. Jaskulek, B. Wilken, The Galileo energetic particle detector. Space Sci. Rev. 60, 385 (1992). doi:10.1007/978-94-011-2512-3_16

D.J. Williams, R.W. McEntire, C. Schlemm II, A.T.Y. Lui, G. Gloeckler, S.P. Christon, F. Gliem, Geotail energetic particles and ion composition instrument. J. Geomagn. Geoelectr. 46(1), 39-57 (1994). doi:10.5636/jgg.46.39 